\title{
Optimal test and sampling designs for polytomous item response theory models
}

Citation for published version (APA):

Lima Passos, V. (2005). Optimal test and sampling designs for polytomous item response theory models. [Doctoral Thesis, Maastricht University]. Maastricht University. https://doi.org/10.26481/dis.20050127vl

Document status and date:

Published: 01/01/2005

DOI:

10.26481/dis.20050127vl

Document Version:

Publisher's PDF, also known as Version of record

\section{Please check the document version of this publication:}

- A submitted manuscript is the version of the article upon submission and before peer-review. There can be important differences between the submitted version and the official published version of record.

People interested in the research are advised to contact the author for the final version of the publication, or visit the DOI to the publisher's website.

- The final author version and the galley proof are versions of the publication after peer review.

- The final published version features the final layout of the paper including the volume, issue and page numbers.

Link to publication

\footnotetext{
General rights rights.

- You may freely distribute the URL identifying the publication in the public portal. please follow below link for the End User Agreement:

www.umlib.nl/taverne-license

Take down policy

If you believe that this document breaches copyright please contact us at:

repository@maastrichtuniversity.nl

providing details and we will investigate your claim.
}

Copyright and moral rights for the publications made accessible in the public portal are retained by the authors and/or other copyright owners and it is a condition of accessing publications that users recognise and abide by the legal requirements associated with these

- Users may download and print one copy of any publication from the public portal for the purpose of private study or research.

- You may not further distribute the material or use it for any profit-making activity or commercial gain

If the publication is distributed under the terms of Article $25 \mathrm{fa}$ of the Dutch Copyright Act, indicated by the "Taverne" license above, 


\section{Optimal test and sampling}

\section{designs for polytomous Item}

Response Theory models

by

Valéria Lima Passos 


\title{
Optimal test and sampling designs for polytomous Item Response Theory models
}

\author{
PROEFSCHRIFT
}

\author{
ter verkrijging van de graad van doctor \\ aan de Universiteit Maastricht, op gezag van de \\ Rector Magnificus Prof. mr. G.P.M.F. Mols \\ volgens het besluit van het College van Decanen, \\ in het openbaar te verdedigen op donderdag \\ 27 januari 2005 om 16:00 uur \\ door
}

Valéria Lima Passos 


\section{Promotor:}

Prof. dr. M.P.F. Berger

\section{Beoordelingscommissie:}

Prof. dr. W.N.J. Groot (voorzitter)

Dr. Tj. Imbos

Prof. dr. W.J. van der Linden (Universiteit Twente)

Dr. ir. A.M.M. Muijtjens

Prof. dr. K. Sijtsma (Universiteit van Tilburg)

ISBN 90-5681-214-9

The Netherlands Organization of Scientific Research (NWO-MAGW) is gratefully acknowledged for funding this project (no. 575-30-009). 


\section{Contents}

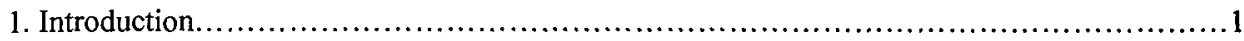

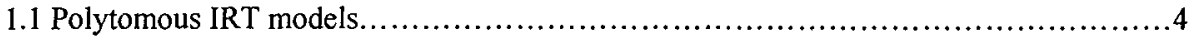

1.1.1 The Nominal Response Model...............................................

1.1.2 The Graded Response Model ...........................................6 6

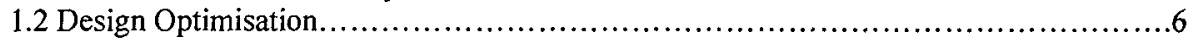

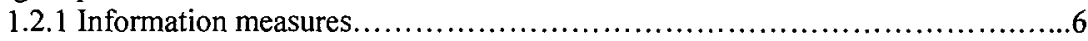

1.2.1.1 Fisher's Information...........................................6

1.2.1.2 Kullback-Leibler Information........................................ 7

1.2 .2 Optimality criteria.........................................................

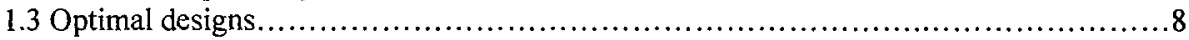

1.3.1 Locally optimal calibration design...................................... 9

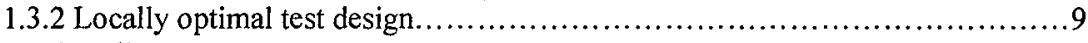

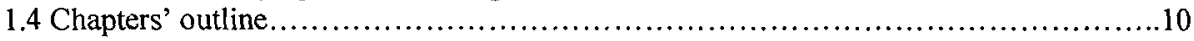

2. Maximin calibration design for the Nominal Response Model: An empirical evaluation........13

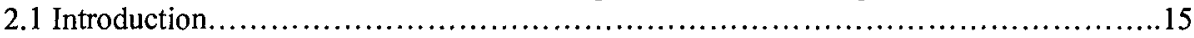

2.2 The Nominal Response Model................................................ 17

2.2.1 Fisher's Information for the NRM ......................................

2.3 Design optimisation...........................................................

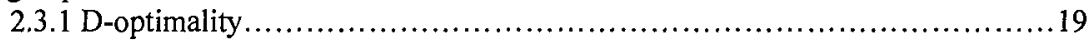

2.3.2 The Maximin approach.............................................. 20

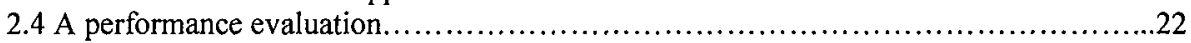

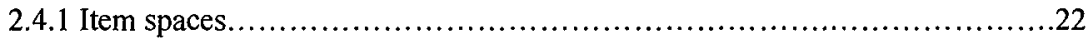

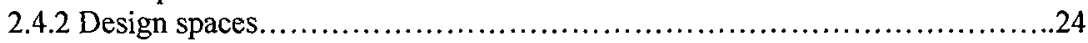

2.4.3 Minimum relative efficiencies..........................................28

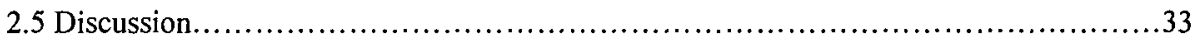

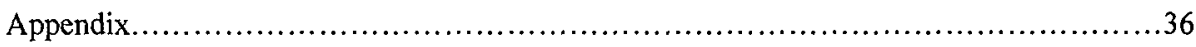

3. Test design optimisation for the Nominal Response Model..............................37

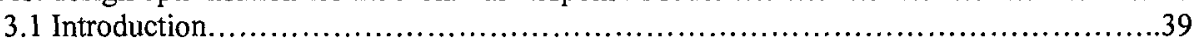

3.2 The Nominal Response Model.................................................40

3.2.1 The NRM............................................................40

3.2.2 Fisher's Information for the NRM.......................................4 41

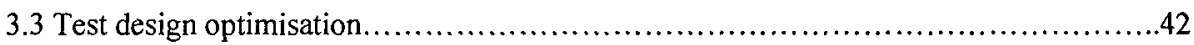

3.3 .1 Optimality criteria.................................................... 44

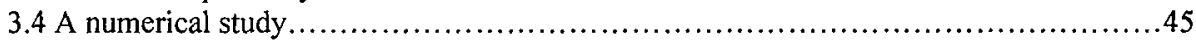

3.4.1 Progress test ............................................................... 45

3.4 .2 Selection of items........................................................ 45

3.4 .3 Optimal test designs..................................................47

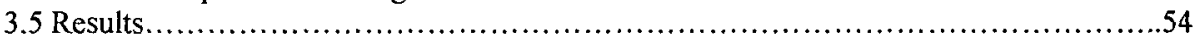

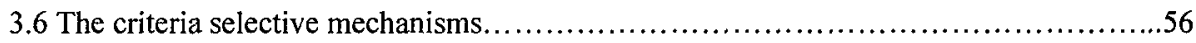

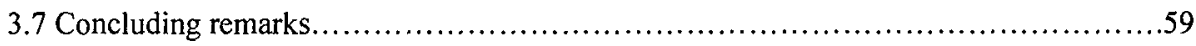

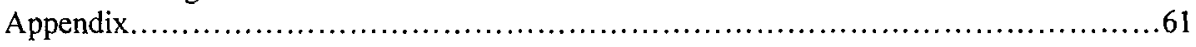

4. Test design optimisation in CAT early stage with the Nominal Response Model............63

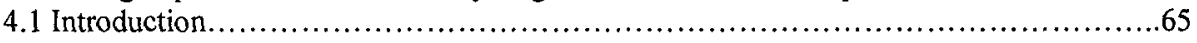

4.2 The Nominal Response Model and Information measures

4.2.1 The NRM...........................................................66

4.2.2 Fisher's Information.......... Universtetion 
4.2.3 Kullback-Leibler Information............................................6

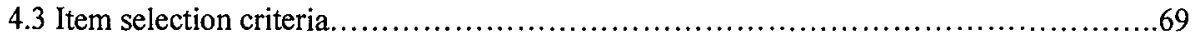

4.4 Part 1 - An early stage investigation................................................. 71

4.4.1 Number of items in the early stage ........................................... 71

4.4.2 Item selection criteria.................................................. 72

4.4.3 Widths of the trait interval.................................................

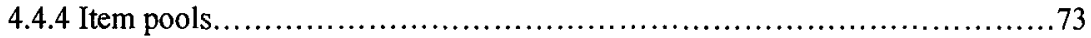

4.4.5 Measuring the criteria performances....................................73

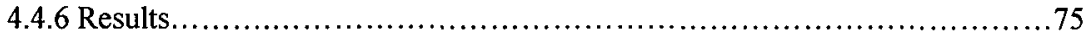

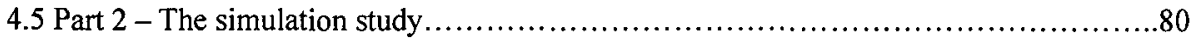

4.5.1 Item selection criteria and trait interval................................... 80

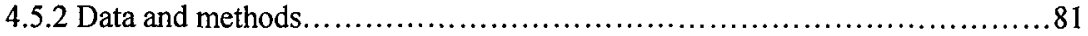

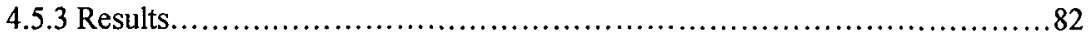

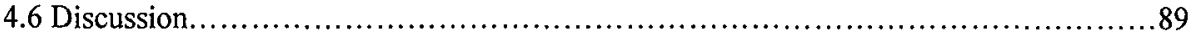

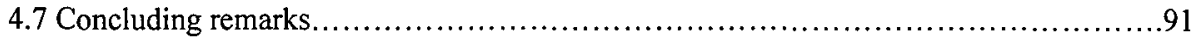

5. D-optimality item selection criterion in CAT early stage - A study with the Graded Response

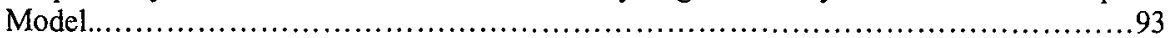

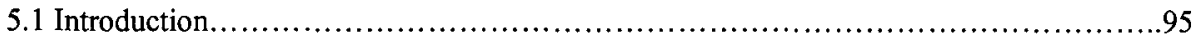

5.2 The Graded Response Model and Information measures.............................97

5.2.1 The Graded Response Model............................................97

5.2 .2 Information Measures................................................... 95

5.3 Item selection criteria......................................................... 99

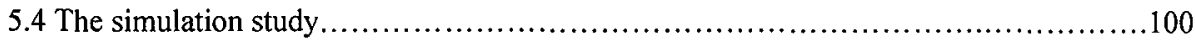

5.4.1 Data and methods.......................................................

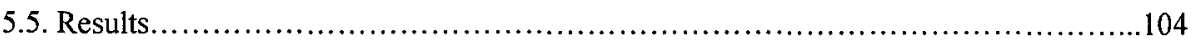

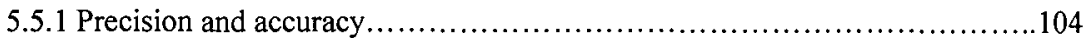

5.5.2 Criteria selective strategies............................................ 109

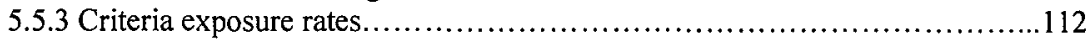

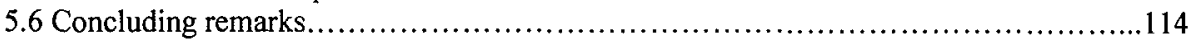

6. On polytomous versus dichotomous items: The quest of information revisited..............117

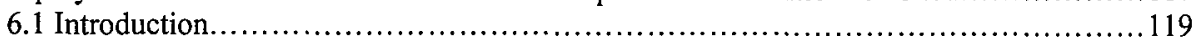

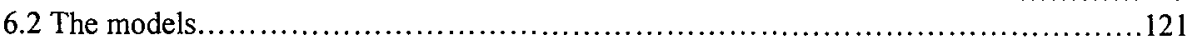

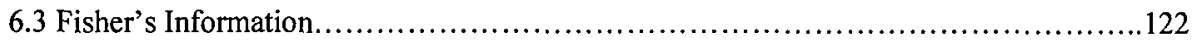

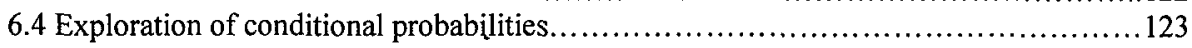

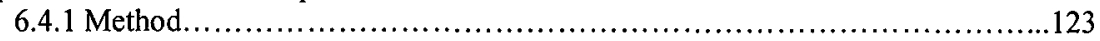

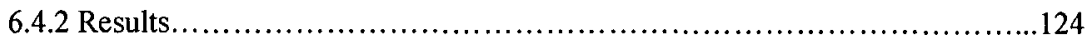

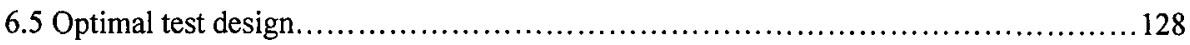

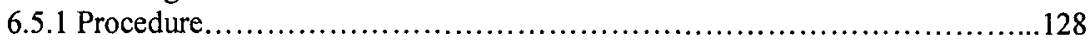

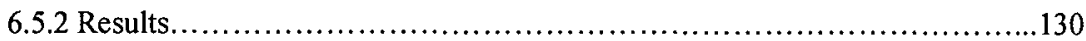

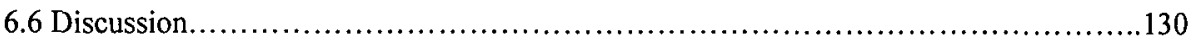

7. Epilogue.................................................................... 135

References...................................................................... 143

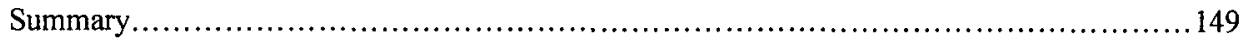

Sammenvatting in het Nederlands..................................................... 153

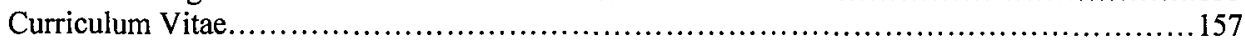

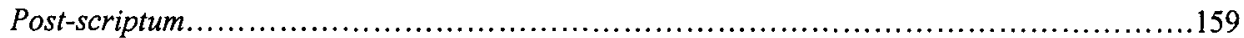


1 Introduction 
Chapter I 


\section{Introduction}

Item Response Theory (IRT) models have become an indispensable tool for the quantification of cognitive and psychological attributes (Hambleton and Swaminathan, 1985, Hambleton, Swaminathan and Rogers, 1991, Baker, 1992, van der Linden and Hambleton, 1997). A few examples of these are abilities, educational achievements, proficiencies, attitudes, personality traits, political inclinations. These non-observable features are generically referred to as latent traits. Instrumental in the measurement of those traits are examinees' test performances, which are observed in form of scored responses on a set of items.

Depending on the number of response alternatives (binary or multiple) items and their descriptive models are categorised into dichotomous or polytomous, respectively. In either case, an IRT model describes the probability of a test-taker to select one of the available item response options conditional and his/her trait level. Traditionally an examinee's response behaviour is assumed to be influenced by a single latent trait, in which case an IRT model is said to be unidimensional. Most of the current IRT applications heavily rely on the assumption of unidimensionality of the trait scale, including the present work. However, advances have been made to relax this assumption, allowing for the multidimensionality of traits underlying psychological and cognitive behaviours to be modelled (Reckase and McKinley, 1991, Veldkamp, 2001).

IRT models can be viewed as non-linear regression models. The slope and intercept parameters define the item's psychometric properties, conveying its discriminative power and level of difficulty, respectively. The independent (non-observable) random variable is the latent trait, representing a person's parameter. Both items and person parameters are estimated on the grounds of data information, which is contained within the string of item responses. This amount of information in the observational/experimental data record is the quantity that considerably affects the estimates' precision and accuracy. With the objective to improve the inferential process, statistical techniques of design optimisation have been applied for the estimation of item and trait parameters of IRT models (Berger, 1991, 1992, 1994(a), 1994(b) 1998; Berger and van der Linden, 1995; Berger and Veerkamp, 1996; Berger and Mathijssen, 1997; Berger, King and Wong, 2000; Buyske, 1998; Holman and Berger, 2001; Wiberg, 2003; Lima Passos et al., 2004).

Simply stated, the objective of optimal experimental design is maximisation of information contained in the data for purpose of estimation. This is accomplished by selecting a set of design points (a grid of values of the independent variable) and finding a distribution 
of observations over these points (experimental design) that results in maximum information for estimation (van der Linden, 1998). For IRT this process is translated into the following two questions: which are the best items to compose a test, in order to efficiently estimate the latent trait? Moreover, which levels of traits are required for best calibrating (estimating) the item parameters? Given two complementary estimation processes, there are correspondingly two cases of design optimisation in IRT. The search of the best sample of test-takers for item calibration is denoted optimal calibration or sampling design, whereas the selection of the best items to compose a test is denoted optimal test design.

Van der Linden (1998) claims that optimal test design is one of four approaches available to find a solution to an optimal test assembly problem. Additional to the former, there are the heuristic-based test assembly (Luecht, 1998 and Sanders and Verschoor, 1998), the 0-1 linear programming (van der Linden and Reese, 1998) and the network-flow programming (Armstrong, Jones and Kunce, 1998). These approaches are equally applicable to item parameter calibration, although the optimal design approach seems to be the most frequently applied (Buyske, 2004). Irrespective of their underlying differences, these approaches share a common denominator, namely the maximisation of an objective function of an information measure, usually Fisher's information.

Optimal test and calibration designs for polytomous IRT models are the central topic of the current thesis. It is the intent of this study to contribute with guiding lines and recommendations for practitioners and test-makers as how to efficiently construct tests and calibrate items.

\subsection{Polytomous IRT models}

In the history of IRT, items responses were initially modelled by a function that allowed only binary outcomes (correct/incorrect, yes/no, positive/negative). The most frequently applied dichotomous models have been the Rasch model, two- and three-parameter logistic models (2PL and 3PL) and probit models (Hambleton and Swaminathan, 1985). Even for test cases, in which scored responses were obtained by multiple alternatives, the ensuing data analysis was eventually undertaken by shrinking the multiple scores into a binary pattern. Polytomous models allow for each response category to be described by its own probability response function, usually referred to as item category characteristic curve (ICCC).

Thissen and Steinberg (1986) proposed a taxonomic system for polytomous models based on the different approaches for estimating the $k_{i}$ alternative response functions of an item $i$. Accordingly two groups can be distinguished: the divided-by-total and the difference 
models. The former require simultaneous estimation of all $k_{i}$ probability functions. The latter replaces simultaneous by subsequent and individual estimation of $\left(k_{t}-1\right)$ cumulative probability functions in a binary fashion (Abrahamowicz and Ramsay, 1992). The core of this thesis is centred on the most basic model from the divided-by-total group, known as the Nominal Response Model (NRM). For comparative purposes and for validation of a technique introduced to the NRM in chapter 3 , chapter 4 also considers a model of the difference taxonomic class, namely the Graded Response Model (GRM).

\subsubsection{The Nominal Response Model (NRM)}

The most basic form of the divided-by-total models is given by the Nominal Response Model (NRM), firstly introduced by Bock (1972). From NRM several other models can be generalised, as the partial credit model (Masters, 1982), the rating scale model from Andrich (1978) and the dichotomous 2PL model. NRM is suitable for modelling items with a nominal response format, requiring no ordering of the response categories. The equation that describes the NRM is:

$$
P_{i g \mid \theta}=\frac{\exp \left(c_{i g}+a_{i g} \theta\right)}{\sum_{g=1}^{k_{i}} \exp \left(c_{i g}+a_{i g} \theta\right)},
$$

where $a_{i g}$ is the discrimination parameter of category $g$ of item $i, i=1, \ldots, n, c_{i g}$ is the transformed location parameter associated with category $g$ of item $i, k_{i}$ is the number of categories of item $i$ with $g=1,2, \ldots, k_{i}, \theta$ is the latent trait variable or ability level of the test-taker, and $P_{i g \mid \theta}$ is the probability of examinee with ability $\theta$ of choosing category $g$ of item $i$, i.e. the probability response function. Frequently the location parameter is denoted $b_{i g}$, where $b_{i g}=-\left(c_{i g} / a_{i g}\right)$. The use of $b_{i g}$ instead of its transformed version $c_{i g}$ is more current for dichotomous and graded response models, since $b_{i g}$ is on the same scale of the latent trait, representing an item's difficulty level. It conveys the location of an item characteristic curve for dichotomous items or of the boundary curve for graded response items on the latent trait continuum. This interpretation is not as straightforward for the NRM, since the location and form of the ICCCs depends upon the combinations of parameter values for the item's full set of response categories (Baker, 1992, p. 255). 


\subsubsection{The Graded Response Model (GRM)}

The Graded Response Model (GRM), introduced by Samejima (1969), belongs to the class of models, for which the responses are measured on an ordinal scale. The response options are ordered with respect to the degree of success to the solution of a problem. The ordering of the response options is a necessary precondition to be met in order to enable estimation of the cumulative probability response functions $P_{i g \theta \theta}^{*}$, individually. The equation that describes a boundary curve is given by:

$$
P_{i g \mid \theta}^{*}=\frac{\exp \left[a_{i}\left(\theta-b_{i g}\right)\right]}{1+\exp \left[\left(a_{i}\left(\theta-b_{i g}\right)\right]\right.}
$$

where $\theta$ is the latent trait variable of an examinee, $a_{i}$ is the discrimination parameter of item $i$, for all the boundary curves $P_{i g \mid \theta}^{*}$, with $i=1,2, \ldots, n, b_{i g}$ is the location parameter associated with boundary curve $g$ of item $i$ with $g=1,2, \ldots, k_{i}$ and $k_{i}$ represents the total number of response categories. To maintain the underlying order of response categories, the $\left(k_{i}-1\right)$ location parameters need to be ordered, $b_{i\left(k_{i}-1\right)}<\ldots<b_{i g}<\ldots b_{i 1}$.

\subsection{Design optimisation}

The selection of an optimal design is based on criteria that envisage maximisation of information contained in the data record. The most commonly applied information measure is Fisher's Information. More recently, the Kullback-Leibler (KL), also known as global information or relative entropy, has been used for optimal item selection.

\subsubsection{Information measures}

\subsubsection{Fisher's Information}

Models' parameters are estimated on the grounds of the information supplied by the observed data on the unknown parameter $\theta$. This quantity is denoted Fisher's information Inf and is defined as the minus expected value of the second derivative with respect of the log likelihood function $\log L$ : 


$$
\operatorname{Inf}=-E\left(\frac{\partial^{2} \log L}{\partial \theta^{2}}\right)
$$

where $L$ is the likelihood of a sample of $n$ independent observations from a probability distribution $f(x \mid \theta)$. The likelihood function is model dependent. In the case of several parameters, gathered in a vector $\Theta=\left[\theta_{1}, \theta_{2}, \ldots, \theta_{s}\right]$, Fisher's information becomes a matrix, whose elements $\operatorname{Inf} f_{k}$ are given by:

$$
\operatorname{Inf}_{j k}=-E\left(\frac{\partial^{2} \log L}{\partial \theta_{j} \partial \theta_{k}}\right),
$$

where $(j, k)=1,2, \ldots$, s. (Everitt, 1998, p.127-128). In IRT, the second derivative of $\log L$ is taken with respect to the parameter to be estimated, either the latent trait or the item parameters.

The ubiquity of Fisher's information in the field of experimental design can be traced back to two of its properties: its dependence on the observed data record and its asymptotic relationship to the inverse of the variance of the maximum likelihood estimator. This means that, choosing a set of observations that maximises an objective function of Fisher's information can considerably improve the inferential process. A way to safeguard an efficient estimation is to mobilise statistical expertise at the design stage, i.e. the stage prior to data collection, allowing for the latter to be optimally executed. By so doing, lavish expenditure of costs may be concurrently avoided.

\subsubsection{Kullback-Leibler Information (KL)}

The KL or global information is defined as an expected value of a ratio of two likelihood functions specified by two parameters $\theta$ and $\theta_{0}$ and measures the discrepancy between them (Everitt, 1998, p.181):

$$
K\left(\theta, \theta_{0}\right)=E\left(\log \frac{L\left(\theta_{0}\right)}{L(\theta)}\right)
$$


The likelihood ratio is commonly applied in the context of hypothesis testing, known as the likelihood ratio test, (Everitt, 1998, p.229), which tests $\theta=\theta_{0}$ versus $\theta=\theta_{1}$, being an optimal approach to hold $\theta_{0}$ and $\theta_{1}$ apart (Chang and Ying, 1996). These latter authors were the first to introduce the expected value of this ratio, to define a global information criterion for item selection (Chang and Ying, 1996).

\subsubsection{Optimality criteria}

In practice there are usually multiple parameters to be estimated: two or more parameters of several items, or a distribution of latent traits, for instance. Consequently, the criteria of optimality are typically defined as an objective function $\Phi($.$) of Fisher's$ Information matrix, $\Phi(I n f)$. Popular functions are the determinant and the trace of this matrix, known as D- and A- optimality criteria, which maximise $\Phi_{\mathrm{D}}(\operatorname{Inf})=\operatorname{det}(\operatorname{Inf})$ and $\Phi_{\mathrm{A}}(\operatorname{Inf})=$ trace(Inf), respectively. These optimality criteria are described at greater length in the individual chapter of this thesis. For further details on these and other criteria based on Fisher's Information within a broader context of optimal experimental design see also Atkinson and Donev (1996).

For item selection, Chang and Ying proposed a criterion for item selection that integrates $\mathrm{KL}$ information of an item $i, K_{i}\left(\theta, \theta_{0}\right)$, over a trait interval $\left[\theta_{\mathrm{L}}, \theta_{\mathrm{U}}\right]$, where $\theta_{\mathrm{L}}$ and $\theta_{\mathrm{U}}$ are the lower and upper interval boundaries, respectively, and the true trait value $\theta_{\mathrm{o}}$ is fixed and known with $\theta_{0} \in \Theta=\left[\theta_{\mathrm{L}}, \theta_{\mathrm{U}}\right]$ (chapter 4):

$$
K_{i}\left(\theta_{0}\right)=\int_{\theta_{L}}^{\theta_{U}} K_{i}\left(\theta, \theta_{0}\right) d \theta
$$

\subsection{Optimal designs}

As to the nomenclature and generic notations: Designs or design measures are usually denoted $\xi$ and are elements of a design space X. Optimal designs, denoted $\xi^{*}$ distinguish themselves from other available designs, $\xi \in \mathrm{X}$, by maximising an optimality criterion based on an information measure. For linear models a design $\xi^{*}$ can be generally found that, according to a chosen criterion, is globally optimal. This means that the design $\xi^{*} \in \mathrm{X}$ will always yield the most efficient estimators irrespective of the values the parameters might take. 
Global optimality does not exist for non-linear models. For the latter, Fisher's information is dependent on the parameter values. Consequently, designs can be found that are optimal for a given set of parameter values, i.e. locally optimal. Different parameters lead to different locally optimal designs. This dependence poses a new problem in the choice of an optimal design, since the parameters are actually not known in the design stage. There are several approaches to handle this issue, among them sequential estimation, Bayesian methods, requiring a prior distribution of parameters to be integrated away, or a maximin method (chapter 2), which requires a range of parameter values to be pre-specified.

\subsubsection{Locally optimal calibration design}

For sampling or calibration designs, the design space is represented by group of testtakers - the trait parameter space $\Theta$. Its elements are the trait levels $\theta_{l}$ of examinees, or design points, with $\theta_{t} \in \Theta$. The vector of trait levels and their corresponding weights determine the design measure $\xi$ :

$$
\xi=\left[\begin{array}{c}
\theta_{1}, \theta_{2}, \ldots, \theta_{t} \\
w_{1}, w_{2}, \ldots, w_{t}
\end{array}\right]
$$

The total number of examinees used for calibration is $N$. The weights represent, thus, the number of respondents with trait $\theta_{j}, j=1,2, \ldots, t$, such that $\sum_{j=l}^{t} w_{j}=N$.

\subsubsection{Locally optimal test designs}

For test designs the design points become the items themselves $i$, and the design space is the item pool $I$, which consisted of calibrated items $i \in I$. For IRT models in this thesis, the statistical properties of a single item are contained in a vector pair of discrimination and location parameters $\left(a_{i}, c_{i}\right)$, where $a_{i}=\left[a_{i 1}, a_{i 1}, \ldots, a_{i g}\right]$ and $c_{i}=\left[c_{i 1}, c_{i 1}, \ldots, c_{i g}\right]$ with $\mathrm{g}=1,2$, $\ldots, k_{i}$, the total number of response categories. A test design measure becomes the set of $n$ items with their corresponding parametric characteristics, where $n$ indicates the test length:

$$
\xi=\left[\left(a_{1}, c_{1}\right),\left(a_{2}, c_{2}\right), \ldots,\left(a_{n}, c_{n}\right)\right]
$$


In test design there are two test settings to be distinguished. The item selection is undertaken envisaging simultaneous estimation of several traits, in which case a group of examinees have to respond to the same items, concurrently selected, i.e. paper and pencil (p\&p) tests (chapter 3). Alternatively, there is the computerised adaptive testing (CAT), in which items are tailored to a single individual, whose trait is sequentially updated as items are selected (van der Linden and Glas, 2000).

\subsection{Chapters' outline}

There is an extensive list of bibliographies on design optimisation in educational and psychological assessment. Among these, literature references on dichotomous models greatly outnumber the ones on polytomous models. The present work can be regarded as an extension of earlier researches on polytomous items, notably Holman and Berger (2001), Berger (1998), Berger and Mathijssen (1997). With its five self-contained chapters, this thesis offers new perspectives and explanations, which underpin practical recommendations, found at the end of each chapter.

Chapter two deals with optimal calibration designs. It describes a maximin approach based on the D-optimality criterion to find optimal designs for estimating NRM item parameters. In the case of estimation of multiple parameters, the D-optimality criterion is rather useful, since it minimises the generalised variance of estimators, i.e. the volume of the confidence ellipsoid of the parameters. A viable strategy to circumvent the problem of local optimality, based on the concept of relative efficiency is presented.

Chapter three applies a sequential algorithm for item selection based on the A- and Doptimality criteria for composing a fixed-form, group-based tests. As in the preceding chapter, items are described by the NRM. Similar work on dichotomous models had been previously undertaken (Berger and van der Linden, 1995). These criteria have been shown to differ in their item selection, but the reasons for the obtained divergences have not been examined in detail. This chapter attempts to explain the criteria modus-operandi as a function of the item pool composition and the underlying trait distribution, upon which maximisation of information is sought. An argument is developed and validated, which claims that knowing in advance the criteria working mechanism allows for a better adjustment between item selection criterion and the test's goals and objectives, usually expressed in form of a target information function (TIF).

Chapter four sets the same item selection criteria of the previous chapter into a framework of computerised adaptive testing (CAT), in which traits are individually estimated 
and items sequentially selected. Chapter four builds upon the results obtained in chapter three, which indicated a greater sensitivity of the D-criterion to the changes on the trait distribution. Additionally to the A- and D-criteria, both based on Fisher's Information, the KullbackLeibler (KL) information criterion is also used for item selection. The performance of the optimality criteria in CAT is examined via a simulation study and the D-criterion's feasibility demonstrated in terms of precision, accuracy and stability of estimation.

Chapter five extends the CAT simulation study to the case of graded response model (GRM). The additional issue of item exposure is considered. Finally, chapter six ventures into a controversy as regards information provided by polytomous and dichotomous items. A general view that assigns to polytomous items greater informative value over dichotomous is partially challenged for tests with nominal response format. In the epilogue (chapter 7), the strength and weakness of the presented methods are revisited and discussed in the light of their potential implications for the practical world. A few suggestions for further research are also presented.

All chapters have been submitted for publication. Chapter 2 was published in Applied Psychological Measurement (Lima Passos and Berger, 2004). Chapter 4 has been reviewed by $A P M$ and is undergoing revision for publication. Chapter 3 and 5 are under review. The bibliographic references have been compiled in a list at the end of the thesis. 
Chapter I 


\section{Maximin calibration designs for the Nominal Response Model: An empirical Evaluation}

This chapter has been published as: Lima Passos, V. and Berger, M.P.F. (2004). Maximin calibration designs for the Nominal Response Model: An empirical evaluation. Applied Psychological Measurement, 28, 72-87. 


\title{
Maximin calibration designs for the Nominal Response Model: An empirical evaluation
}

\begin{abstract}
The problem of finding optimal calibration designs for dichotomous IRT models has been extensively studied in the literature. In the present paper this problem will be extended to polytomous IRT models. Focus is given to items described by the Nominal Response Model (NRM). The optimisation's objective is to minimise the generalised variance of item parameter estimates. For non-linear models, Fisher's Information depends on the unknown parameters to be estimated. By assuming knowledge of the latter, D-optimal designs can be found, however, with the inconvenience that these are only locally optimal. A modified maximin approach, based on the relative efficiency of calibration designs with respect to the D-optimal design, is introduced as a viable method to circumvent the problem of local optimality. An algorithm for finding a maximin design is described. The performance of maximin designs is compared to that of alternative calibration designs, those with normally and uniformly distributed latent trait values.
\end{abstract}

Keywords: Polytomous IRT Models, Item Calibration, Maximin Design, Relative Efficiency 


\section{Maximin calibration designs for the Nominal Response Model: an empirical evaluation}

\subsection{Introduction}

Several Item Response Theory (IRT) models have been developed and increasingly applied in the field of psychological and educational measurement. The most often used IRT models, the one-, two- and the three-parameter logistic models, describe the probability of an examinee, conditional on his/her ability, to choose a correct answer to a dichotomous item. However, in the last decades growing interest has arisen in items with multiple response categories, denominated polytomously scored items (Drasgow, 1995).

In the past, the evaluation of tests with polytomous items used to be carried out by shrinking the multiple alternative answers into dichotomous scores. The results were eventually analysed with dichotomous models. The development of polytomous models put an end to this mismatch between model and data. These models make it possible for each response option to be described by its own category characteristic function, resulting in a gain in information on the latent trait (Bock, 1972; Thissen, 1976). An overview of polytomous models can be found in Thissen and Steinberg (1986) and Dodd, Ayala and Koch (1995).

One of the purposes of a constructed item is to quantitatively estimate a latent trait variable, a psychological trait. Items are constructed, calibrated and kept in item banks, where they can be selected for test assembly. The selection of a test's constituent items takes place on the grounds of the items' content, as well as on their parametric characteristics. These are a function of the item's power of discriminating among examinees trait levels, and of its degree of difficulty, known also as the item dispersion and location parameters, respectively.

Two complementary estimation processes, namely estimation of traits via test assembly, and estimation of item parameters via calibration, give rise to the two cases of design optimisation within the context of IRT. The first case is the optimal test design, in which optimisation is targeted over a space of item parameters, envisaging efficient trait estimation. The second case relates to optimal sampling or optimal calibration design, in which optimisation is sought over a space of traits, in order to obtain efficient item parameters estimates. The focus of this paper is centred on optimal calibration design. 
There is an extensive literature on calibration of dichotomously scored items (Stocking, 1990; Berger, 1991; 1992; 1994a; Jones and Jin, 1994; Buyske, 1998; Berger, King and Wong, 2000; Jones and Nediak, 2000). In contradistinction, up to the present day, little research has been done with respect to calibration designs for polytomous items. So far, one work on this subject matter can be cited (Holman and Berger, 2001), which is rather technical in its content. These authors have shown, among others, that the sequential search of an optimal design, which has been successfully used for calibration of dichotomous models (Berger, 1992, 1994, Jone and Jin, 1994), is also valid for polytomous IRT models. The need for more research, however, is required to provide guidelines to practitioners for using optimal calibration designs for items with multiple response categories.

As a whole, the present study can be regarded as an extension of the former. It is, however, autonomous and introduces an approach that is slightly more tuned into practical questions. Its objective is to present a method, which facilitates the search of an optimal calibration design for polytomous items. To attain this goal, statistical methods from optimal design theory (Atkinson and Donev, 1996) will be applied in connection with Fisher Information on the item parameters of the Nominal Response Model (NRM).

In design optimisation, one of the greatest problems for IRT models is the dependence of Fisher's information on the values of the model parameters. This dependence does not allow for the existence of globally optimal designs. This is to say that a single design cannot be found that, irrespective of which values the estimable parameters might take, always offers the maximum information on them. For non-linear models, only local optimality can be achieved, i.e. the design is optimal for a given set of parameter values.

The problem of local optimality can be circumvented by introducing a maximin approach. The idea is to compare performances of different local optimal designs for several values of item parameters. Eventually, one design is to be selected, which best meets the demand of 'minimising the highest loss in relative efficiency, i.e. a maximin design. The relative efficiency of the maximin design will be compared with normal random sampling and uniform designs. Finally, considering the large numbers of estimable discrimination and location parameters in polytomous models, an analytical solution of optimal designs becomes unfeasible. As a result, this work relies heavily on numerical methods. 


\subsection{The Nominal Response Model (NRM)}

The model to be dealt with in this paper, the Nominal Response Model (NRM), was firstly introduced by Bock (1972). The NRM is considered the most general model in the divide-by-total model classification category (Thissen and Steinberg, 1986). It does not require the ordering of categories to reflect different degrees of the trait level to be measured. It is, therefore, suitable for nominal data (Heinen, 1996, p.103). This characteristic stands in opposition to other IRT polytomous models, like the rating scale model (Andrich, 1978), the partial credit model (Masters, 1982).

The equation that describes the NRM is:

$$
P_{i g \mid \theta_{t}}=\frac{\exp \left(c_{i g}+a_{i g} \theta_{t}\right)}{\sum_{h=1}^{k_{i}} \exp \left(c_{i h}+a_{i h} \theta_{t}\right)},
$$

where $a_{i g}$ is the discrimination parameter of category $g$ of item $i, i=1, \ldots, n, c_{i g}$ is the location parameter associated with category $g$ of item $i, k_{i}$ is the number of categories of item $i$ with $g$ $=1,2, \ldots, k_{i}, \theta_{t}$ is the latent trait variable, and $P_{i g \mid \theta_{t}}$ is the probability of examinee with trait $\theta_{t}$ of choosing category $g$ of item $i$. The traits $\theta_{t}$ are elements of the trait parameters space $\Theta$, i.e. $\theta_{l} \in \Theta$.

\subsubsection{Fisher Information Matrix for the NRM:}

Fisher's Information for item parameters of a single item $i$ is represented by a matrix here denoted $\operatorname{Inf}\left(\xi, a_{i}, c_{i}\right)$. This notation is meant to emphasise the dependence of Fisher's Information on the unknown item parameters as well as on what is described as the design measure $\xi$, to be explained at greater length below.

Both $a_{i}$ and $c_{i}$ represent parameter vectors related to categories $g$ and $h$ of an item $i$, with $a_{i}=\left[a_{i 1}, a_{i 2}, \ldots, a_{i k_{i}}\right], c_{i}=\left[c_{i 1}, c_{i 2}, \ldots, c_{i k_{1}}\right]$, and $(g, h)=1,2, \ldots, k_{i}$. The diagonal and offdiagonal elements of $\operatorname{Inf}\left(\xi, a_{i}, c_{i}\right)$ are given by the minus expectation of the second derivatives of the log likelihood function with respect to the item parameters: 


$$
\operatorname{Inf}_{i}\left(\xi, a_{i}, c_{i}\right)=-E\left[\begin{array}{ll}
\frac{\partial^{2} \log L}{\partial a_{i g} \partial a_{i h}} & \frac{\partial^{2} \log L}{\partial a_{i g} \partial c_{i h}} \\
\frac{\partial^{2} \log L}{\partial c_{i g} \partial a_{i h}} & \frac{\partial^{2} \log L}{\partial c_{i g} \partial c_{i h}}
\end{array}\right]
$$

The specific formulas of the second derivatives of the log-likelihood with respect to the item parameters for the NRM with discretised trait values $\theta_{l} \in \Theta$ can be found in Bock (1972). The part of the $\log$ likelihood function $L$ that needs to be maximised in order to obtain the maximum likelihood (ML) estimates is given by:

$$
\log L=\sum_{t} \sum_{i} \sum_{g} f_{i t g} \log P_{i g \mid \theta_{t}}
$$

where $f_{i t g}$ is the number of examinees with trait $\theta_{t} \in \Theta$ that respond to each of the $k_{i}$ categories of item $i$. The probability $P_{i g \mid \theta_{I}}$ is defined in equation (1).

For the ML estimation of item parameters, certain constraints must be imposed on the values of discrimination and location parameters. They are introduced to avoid problems of identification. In this paper, a constraint will set $a_{i 1}=c_{i 1}=0$, for all $i$, and the categories will be ordered according to their discrimination values, i.e. $a_{i 1}<a_{i 2} \ldots<a_{i g}$ (for a discussion, see Heinen, 1996, p.102). With the application of this constraint, Fisher Information matrix $\operatorname{Inf}\left(\xi, a_{i}, c_{i}\right)$ for the $k_{i}$ categories of item $i$ becomes a $\left(2 k_{i}-2\right) \times\left(2 k_{i}-2\right)$ symmetric matrix.

The discrete design measure $\xi$ in $\operatorname{Inf}\left(\xi, a_{i}, c_{i}\right)$ expresses the dependence of Fisher's information on the assembly of trait points and their respective weights:

$$
\xi=\left[\begin{array}{c}
\theta_{1}, \theta_{2}, \ldots, \theta_{t} \\
w_{1}, w_{2}, \ldots, w_{t}
\end{array}\right]
$$

where $\theta_{1}, \theta_{2}, \ldots, \theta_{l}$ represents a vector of $t$ levels of a unidimensional trait $\theta$. This vector of trait levels is linked to its vector of associated weights, $w_{1}, w_{2}, \ldots, w_{1}$ to build the design measure. The total number of examinees used for calibration is $N$. The weights represent, thus, the number of respondents with trait $\theta_{j}, j=1,2, \ldots, t$, such that $\sum_{j=1}^{t} w_{j}=N$. 


\subsection{Design Optimisation}

\subsubsection{D-optimality}

The objective of design optimisation is to find an optimal design measure $\xi^{*}$, i.e. a vector of optimal trait levels with their corresponding weights, which maximises an optimality criterion over $\Theta$. The selected function of the Information matrix to be optimised is the determinant, $\operatorname{Det}\left[\operatorname{Inf} i\left(\xi, a_{i}, c_{i}\right)\right]$. This determinant or D-optimality criterion has been chosen because of a few advantages it offers. Among others, one of most convenient aspects of the D-optimality criterion is the existence of an inverse relation between the volume of the confidence ellipsoid of the parameters estimates and the determinant of the information matrix. This is so because, asymptotically, the latter is equivalent to the determinant of the inverse of the variance-covariance matrix of parameter estimates $\operatorname{Var}\left(\xi, a_{i}, c_{i}\right)$. The determinant of the variance-covariance matrix, $\operatorname{Det}\left[\operatorname{Var}\left(\xi, a_{i}, c_{i}\right)\right]$, is also known as the generalised variance of the model parameters. By taking both variance and covariance of parameters into account, the determinant can be regarded as a summary measure of the whole matrix $\operatorname{Var}\left(\xi, a_{i}, c_{i}\right)$. A further useful characteristic of the D-optimality criterion, which often earns him prominence among other optimality criteria, relates to its invariance with respect to reparameterisation or linear transformation of the model parameters. Donev and Atkinson (1996, p. 116-119) discuss in more details other aspects of D-optimality.

The objective of the present work is to apply methods of design optimisation in order to minimise the generalised variance, or alternatively, maximise of the determinant of the information matrix. The process of optimising the estimation of item parameters under the Doptimality criterion is translated into selecting the amount and levels of a latent trait from their range of values, which best fulfils the demands of maximising the determinant of the information matrix $\operatorname{Inf}\left(\xi, a_{i}, c_{i}\right)$.

Given the premises of known item parameters, the $\operatorname{Det}\left[\operatorname{In} f_{i}\left(\xi, a_{i}, c_{i}\right)\right]$ can be maximised by selecting a D-optimal design $\xi^{*} \in X$, where $X$ represents a space of all designs, such that for each design $\xi \in \mathrm{X}$ :

$$
\operatorname{Det}\left[\operatorname{Inf}_{i}\left(\xi^{*}, a_{i}, c_{i}\right)\right] \geq \operatorname{Det}\left[\operatorname{Inf}_{i}\left(\xi, a_{i}, c_{i}\right)\right]
$$

In general, a design, which is optimal over the whole parameters space, cannot be found for IRT models. Global optimality cannot be achieved because of the dependence of 
the information matrix on the unknown parameters. However, optimality can be obtained locally for a given set of parameters.

Let us assume the existence of a discrete item space $I$, i.e. a finite space whose elements are $n$ items with fixed combinations of parameters values (the ranges from which the parameter values will be taken are described in more details later). A local D-optimal design $\xi^{*}$ can be found for each constituent item $i \in I$, for which the $\operatorname{Det}\left[\operatorname{Inf}\left(\xi, a_{i}, c_{i}\right)\right]$ is maximised over the values $\theta_{t} \in \Theta$. This can be done sequentially over the discretised trait parameters space, $\Theta$, delimited to vary herein between $[-3,3]$. The sequential algorithm for finding the local D-optimal design for dichotomous items has been elsewhere described (Berger, 1992). Holman and Berger (2001) have demonstrated that this methodology is also applicable to polytomous models. Once this maximisation procedure is carried out, a subset of a designs space $\mathrm{X}^{*} \subset \mathrm{X}$ emerges, where $\mathrm{X}^{*}$ is the set of local D-optimal designs $\xi_{i}^{*}$ for items $i \in I$. The space $\mathrm{X}^{*}$ indicates explicitly the space of $\mathrm{D}$-optimal designs for items of the chosen item space. It should be emphasised that, in this work the designs are optimal with respect to a single item, and not for a set of items, simultaneously.

\subsubsection{The Maximin approach}

In questions of optimisation, it is not unusual to come across maximin or alternatively, minimax rules of thumb. They represent a helpful tool in decision-making situations, where the selection of one among several alternatives is required. These rules are criteria commonly applied with the objective to safeguard against very poor outcomes. In the specific case portrayed in this work, one calibration design is sought, which, after undergone the maximin procedure, will for sure not perform any worse than a certain performance threshold. This performance threshold is to be maximised. In this study, the maximin procedure is described for calibration of items $i \in I$.

For each item $i$ in the discretised item space $I$, there is a corresponding local Doptimal design $\xi^{*}$ in the finite design space $X^{*}$. The availability of several local D-optimal designs in $\mathrm{X}^{*}$ raises the question of how to determine the most adequate sampling to calibrate all items in $I$. Sought is a design $\xi^{\text {maximin }} \in \mathrm{X}^{*}$, for which the incurred loss of efficiency will be minimal for all combinations of item parameters values in $I$. The efficiency loss of design $\xi_{j}^{*}$, once applied to item $i$, is measured by its relative efficiency with respect to the D-optimal design for item $i$ : 


$$
\operatorname{eff}\left(\xi_{j}^{*}\right)=\left(\frac{\operatorname{Det}\left[\operatorname{Inf}\left(\xi_{i}^{*}, a_{i}, c_{i}\right)\right]}{\operatorname{Det}\left[\operatorname{Inf} f_{i}\left(\xi_{i}^{*}, a_{i}, c_{i}\right)\right]}\right)^{\frac{1}{p}}
$$

where $\operatorname{Inf} f_{i}$ is the Information matrix of item $i, i$ and $j$ are indexes for the constituent items of the item space $I,(i, j)=1, \ldots, n, \xi_{j}^{*}$ is the local D-optimal design of item $j$, whose performance is measured with respect to the local D-optimal design $\xi_{i}^{*}$ for item $i$, and $p$ is the number of independently estimable parameters in the model, i.e. $p=\left(2 k_{i}-2\right)$.

The maximin design $\xi^{\text {maximin }}$ is one whose minimum relative efficiency over the space of items is maximised over the space of D-optimal designs $\mathrm{X}^{*}$. Its maximin value $M M V$ is:

$$
M M V=\max _{\mathrm{X}^{*}}\left[\min _{I} e f f\left(\xi_{j}^{*}\right)\right] .
$$

The procedure of minimisation followed by maximisation forces the chosen design to perform with the least loss of efficiency among all possible combinations of parameters, as defined by the items in $I$. The maximin design represents, thus, a 'lower boundary' design for estimating the items $i \in I$, i.e. the worst-case scenario for this specific design. This is to say that, apart from the item that is associated with $M M V$, the application of the maximin design to all others $i \in I$ will obligatory yield higher efficiency values. A detailed description of the developed maximin algorithm can be found in the appendix.

A similar maximin method has been applied to dichotomous IRT models by Berger, King and Wong (2000). However, these authors worked with the absolute, instead of the relative efficiency. Contrary to the relative efficiency, in which the values of determinants are standardised, by taking D-optimality as reference, the absolute efficiency targets the raw determinant value. These different ways of measuring a design performance may lead to different maximin designs, which are not necessarily comparable with each other. Consequently, a decision in terms of selecting the most appropriate design is not possible. They are different approaches, but equally reasonable. Yet, as Ouwens et al. (2002) shows for generalised linear mixed models, using relative over absolute efficiency has advantageous practical implications. The resulting value of any design's relative efficiency can be interpreted in terms of how much its vector of weights needs to be changed, i.e. how many 
more observations need to be added to it, in order to achieve the same efficiency as that of an optimal design.

Within the present context of item calibration, for instance, a relative efficiency of 0.8 for a design $\xi$ with respect to the locally D-optimality $\xi^{*}$ means that $(1 / 0.8-1) 100 \%=25 \%$ more respondents would be necessary to achieve the same efficiency of estimates as the Doptimal design. Note that 0.8 gives the relative efficiency of design $\xi$ with respect to $\xi^{*}$. The other way round, i.e. $1 / 0.8$, the relative efficiency of $\xi^{*}$ with respect to $\xi$ is obtained, which is in this case 1.25 . This is the same as to say that the optimal design $\xi^{*}$ is $25 \%$ more efficient than $\xi$. Since any design is primarily characterised by its support points (the vector of trait levels) any change on the former with the intent to increase efficiency, would automatically change the design. By contrast, a change on the vector of weights, proportional to the already pre-existent distribution of weights associated with the support points, keeps the design's structure intact. Only the size of the calibration sample is affected.

This interpretable comparison in the form of 'extra numbers of examinees' required is not viable, when absolute efficiency is applied, as its was the case of the work of Berger, King and Wong (2000). Furthermore, in the latter work, calibration of items was approached within a simultaneous framework. In the present paper, the design is locally D-optimal for a single item and not several of them simultaneously. The presented algorithm can be easily changed to accommodate the simultaneous nature of items calibration. In this case, a major alteration would involve Fisher's information Matrix, which is defined in (2) for a single item $i$. This matrix would need to be enlarged, becoming a block diagonal matrix, whose diagonal elements are the information matrices $\operatorname{In} f_{f}\left(\xi, a_{i}, c_{i}\right)$ of the $m$ single items, $m$ being the number of items that need to be simultaneously callibrated.

\subsection{A Performance Evaluation}

\subsubsection{Item Spaces}

In the previous section, the core idea of the maximin approach was presented for the case of an items space $I$ with fixed combinations of parameter values. For the purpose of evaluating how the composition of an item space will interfere with the performance of the maximin approach, this work is based on examples of nine item spaces $I_{z}$, with $z=1, \ldots, 9$. All of them have the same number of items $n=600$, which are described by the $g=3$ categories NRM. Table 1 depicts the characteristics of the nine item spaces. These discretised item 
spaces contain a relatively large number of items $(n=600)$ in order to allow for diverse combinations of parameter values to be taken into account.

Table 1. Nine item spaces of varied compositions for the pair combinations $\left[\begin{array}{ll}a_{i 2} & a_{i 3}\end{array}\right]$, with $a_{i 1}=0$.

$$
\left[d_{\min }, d_{\max }\right]
$$

$$
\left[\min \left(a_{i 2}\right) \max \left(a_{i 3}\right)\right] \quad[0.15,0.5] \quad[0.71,2.0] \quad[0.15,2.0]
$$

[ $\left.\begin{array}{lll}0.25 & 3.0\end{array}\right]$

$\begin{array}{lll}I_{1} & I_{2} & I_{3} \\ I_{4} & I_{5} & I_{6} \\ I_{7} & I_{8} & I_{9}\end{array}$

Note: The minimum and maximum interval between the two discrimination parameters is given by $\left[d_{\min }, d_{\max }\right]$. Their limits are given above, in the columns, except for item spaces $I_{2}$ and $I_{3}$, for which they are $[0.15,1.0]$ and $[0.15,1.55]$, respectively.

As it can be seen in Table 1, the differences among the item spaces are associated not only with the selected ranges of discrimination values of the two independently estimable categories. Consideration is also given to proximity of these categories' curves.

There were two ways of systematically varying the composition of the finite and discrete item space: Firstly, by delimiting the width of the ranges, from which fixed combinations of difficulty and discrimination parameters were selected; and secondly, by forcing the values of $a_{i 2}$ and $a_{i 3}$ to lie closer or farther apart from each other. For descriptive purposes, the arithmetic difference between these values is expressed in terms of minimum allowed distance $d_{\min }$. Analogously, a maximum distance $d_{\max }$ can also be imposed in the fixed pair-combinations of discrimination parameters. Determining which $d_{\min }$ and $d_{\max }$ distances are allowed, enables a better control over the problem of items implausibility. The interplay between these two impositions determined the final composition of the item spaces presented in Table 1.

For item spaces $I_{1}, I_{2}$, and $I_{3}$ the total range of discrimination values is shortened, whereas in item spaces $I_{1}, I_{4}$, and $I_{7}$ the values of $a_{i 2}$ and $a_{i 3}$ are forced to lie closer to each other. Note that not only the corresponding $d_{\min }$, but also the $d_{\max }$ is relatively small. By contrast, a different constraint has been imposed on $I_{5}$ and $I_{8}:$ a $d_{\min }$ of at least 0.71 separated 
both $a_{i 2}$ from $a_{i 3}$. As a result, the last two categories curves of the items in $I_{5}$ and $I_{8}$ are reasonably apart from each other in their discriminative power. In fact, for $I_{5}$ this is valid for all three categories due to its range delimitation. In association with Table 1, it should be noted that the variability of parameter combinations of the item spaces increase in the direction up $\rightarrow$ down, left $\rightarrow$ right, $I_{1}$ containing the most homogeneous and $I_{9}$ the most heterogeneous blend of parameters.

As far as the range of location parameters values $c_{i g}$ is concerned, two cases have been differentiated. For $C A S E 1$, the location parameters values $c_{i g}$ have been made to vary between $[-1,1]$. For CASE 2 , this range has been extended to $[-3,3]$. It should be emphasised that for both cases, the variations of the discrimination parameters values occur under the specifications presented by Table 1, resulting in nine different item spaces for each case. For simplicity's sake, the $z$ index of the items spaces $I_{z}$ for both cases run from 1 to 9 , to comply the delimited conditions and notations presented in Table 1.

For illustrative purposes, Table 2 shows the 30 discrimination and 20 location parameter values that have been combined to give rise to the 600 constituent items of item pool $I_{8}$.

\subsubsection{Design Spaces}

This study concentrates on D-optimal designs. For all of the $n=600$ items in an item space $I_{z}(z=1, \ldots, 9)$, locally D-optimal designs $\xi^{*}$ can be found, which together form the corresponding D-optimal design spaces $\mathrm{X}_{z}^{*}, z=1, \ldots, 9$. Herein, the maximin design $\xi^{\text {maximin }}$ is one of the D-optimal designs in $\mathrm{X}_{z}^{*}$.

A few alternative sampling designs have been also considered. The extra, discrete designs are the normal random seeding, $\xi^{\text {nor }}$, the uniform design $\xi^{\text {!ni }}$, a three-points uniform design $\xi^{3-u n i}$ and a five-points uniform design $\xi^{\xi-u n i}$.

The first two designs, $\xi^{\text {nor }}$ and $\xi^{\text {gni }}$, have been taken into account in order to enable a discriminative comparison between a D-optimal maximin design, $\xi^{\text {maximin }}$, and other common calibration designs. The same is valid for $\xi^{- \text {-uni }}$ and $\xi^{\xi^{-u n i}}$. However, different reasons account for their consideration. 
Table 2. An illustration of item parameter values for pool $I_{8}$. The 600 items $i \in I_{8}$ resulted from the combination of each row $\left[\begin{array}{lll}a_{i 1} & a_{i 2} & a_{i 3}\end{array}\right]$ with all $\left[\begin{array}{lll}c_{i 1} & c_{i 2} & c_{i 3}\end{array}\right](30 \times 20)$.

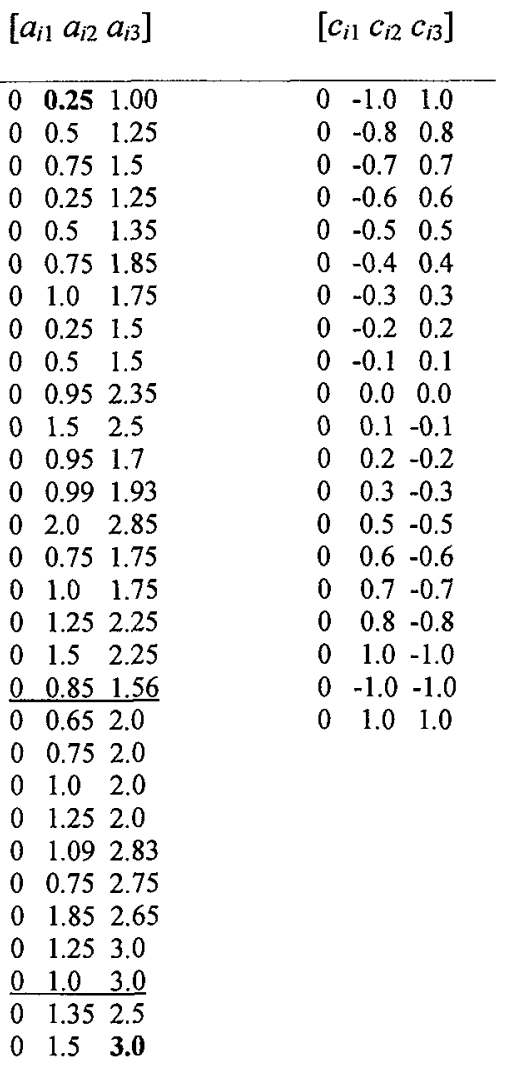

The numbers in bold give the $\min \left(a_{i 2}\right)$ and $\max \left(a_{i 3}\right)$, while the underlined $a_{i g}$ combinations bear $\left[d_{\min }, d_{\max }\right]$ for item pool $I_{8}$.

The first two designs, $\xi^{\text {gor }}$ and $\xi^{\prime \prime \prime}$, have been taken into account in order to enable a discriminative comparison between a D-optimal maximin design, $\xi^{\text {maximin }}$, and other common calibration designs. The same is valid for $\xi^{- \text {-uni }}$ and $\xi^{-u n i}$. However, different reasons account for their consideration. 

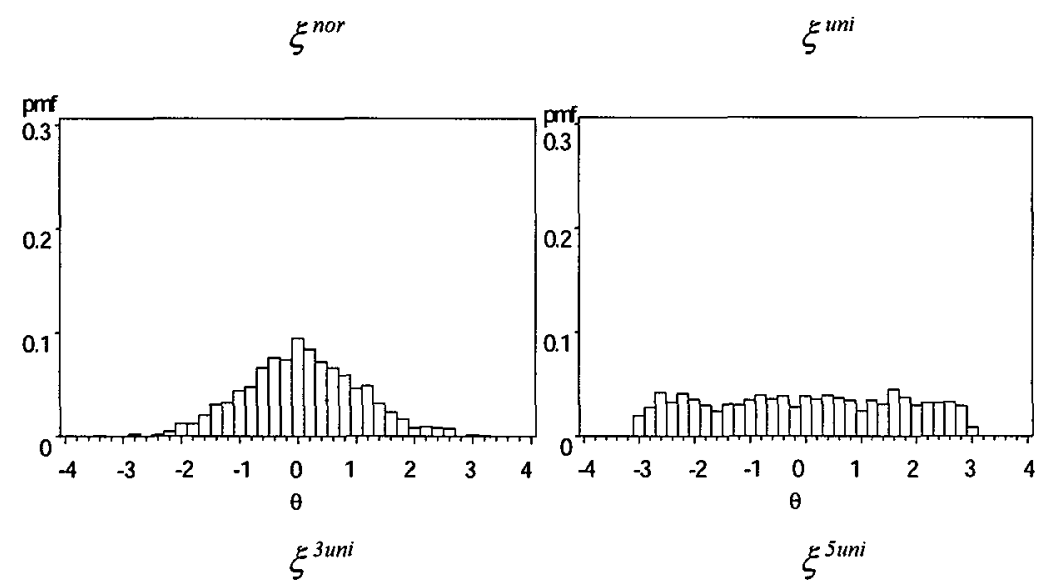

$\xi^{5 u n i}$
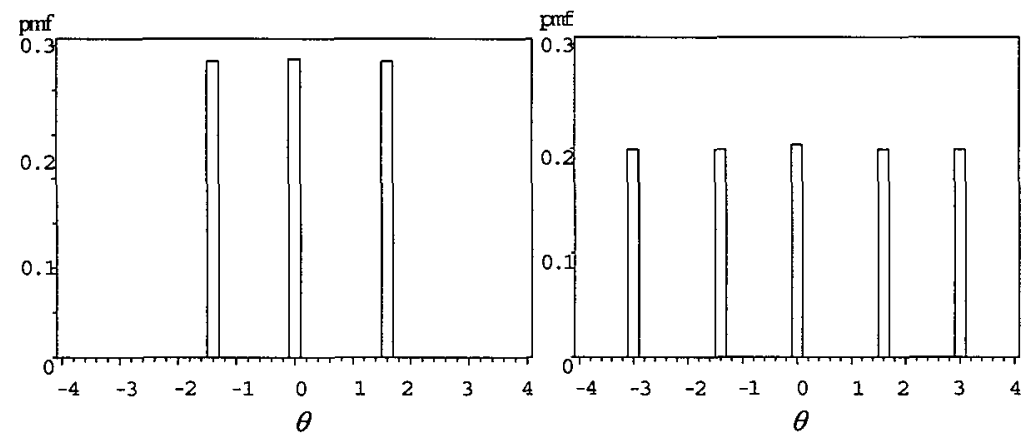

Figure 1. Probabilty mass functions (pmf) for the four alternative designs.

According to a theorem postulated by Holman and Berger (2001), the required number of support points of the latent trait variable for the D-optimal design equals at most the value of $\left(p^{*}+1\right)$, where $p^{*}$ is the number of independently estimable discrimination parameters. Since we are dealing with the $g=3$ categories NRM, the majority of the Doptimal designs $\xi^{*} \in \mathrm{X}^{*}$ for the items in $I_{z}$ have at most three support points. In the face of this general rule, a three-points uniform design $\xi^{-u n i}$ has been considered as an alternative design. Among an infinite variety of three-points designs that can be built along the $\theta$ scale, $\xi^{3-u n i}$ has been devised in such a way as to consist of three points centred on zero and equidistantly spaced along the delimited $\theta$ scale. Furthermore, these points are also equally weighted. In this way, a symmetric and balanced spatial configuration of the design points is attained. The choice of this design is greatly associated with the findings of Ouwens et al. (2002). Given symmetry and compactness of the parameters space, upon which the 
optimisation algorithm will run, Ouwens et al. (2002) have shown that balancedness and symmetry are factual characteristics of

Item Space $I_{l}$

Item Space $I_{2}$

Item Space $I_{3}$

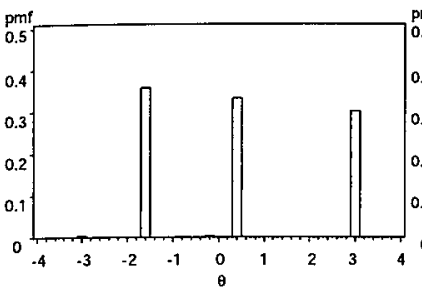

Item Space $I_{4}$

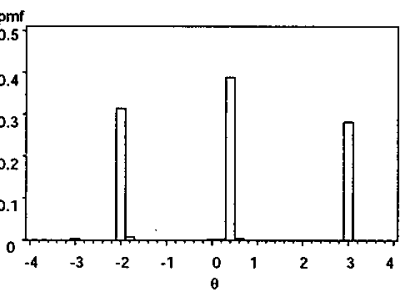

Item Space $I_{5}$

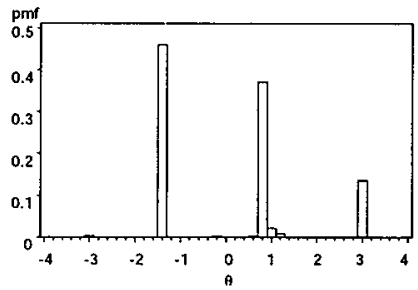

Item Space $I_{6}$

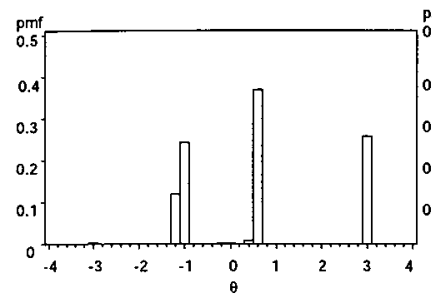

Item Space $I_{7}$

Item Space $I_{8}$

Item Space $I_{9}$

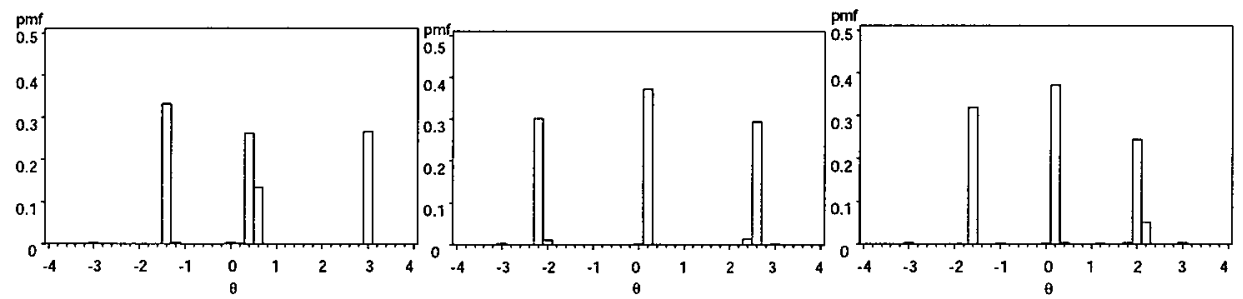

Figure 2. Probability mass functions (pmf) for the nine Maximin D-optimal designs for case 1 .

maximin designs. Similarly to $\xi^{\beta-u n i}$, a balanced and symmetric distribution of support points composes the five-points uniform design $\xi^{-u n i}$. The latter has been introduced as a controlling measure, that together with $\xi^{\text {gor }}$ and $\xi^{\prime n i}$ might indicate whether a different and more efficient design is likely to be expected outside the set represented by the three-point designs. Figures 1 shows the probability mass functions of the designs $\xi^{n o r}, \xi^{\text {gni }}, \xi^{3-u n i}$, and $\xi^{-}$ ${ }^{u n i}$. Figures 2 and 3 , on the other hand, displays the probability mass functions for the maximin designs $\xi^{\text {maximin }}$ for each of the item spaces $I_{z}(z=1, \ldots, 9)$ for CASE 1 and CASE 2, respectively. These maximin designs should have theoretically at most three support points, 
i.e. three major bars. The smaller bars are an artifact resulted from the sequential algorithm for finding local D-optimal designs.

Item Space $I_{I}$

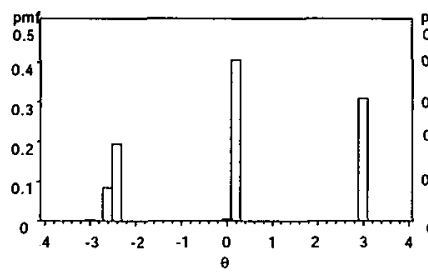

Item Space $I_{4}$

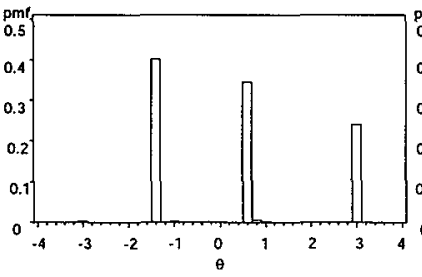

Item Space $I_{7}$
Item Space $I_{2}$

Item Space $I_{3}$

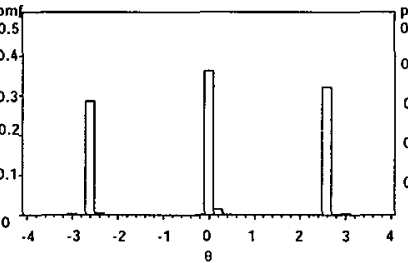

Item Space $I_{5}$

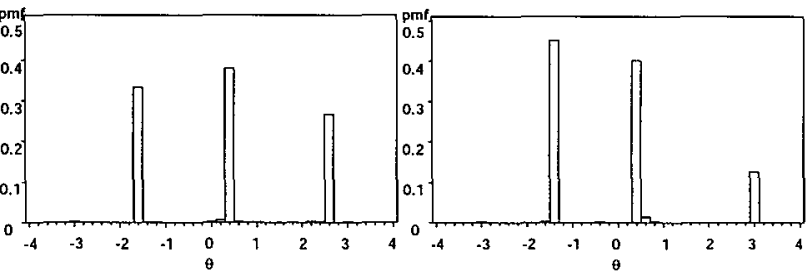

Item Space $I_{9}$

Item Space $I_{8}$

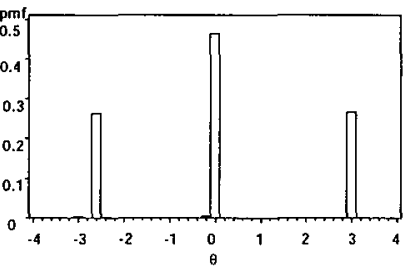

Item Space $I_{6}$

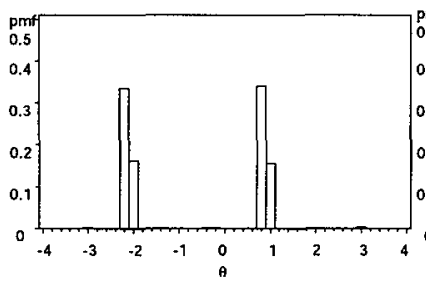

Figure 3. Probability mass functions (pmf) for the nine Maximin D-optimal designs for case 2.

\subsubsection{Minimum Relative Efficiencies}

In Table 3 , the minimum relative efficiencies $\left[\min _{i} \operatorname{eff}\left(\xi_{j}^{*}\right)\right]$ for each of the alternative designs for $C A S E 1$ are displayed. Here, the maximin value $M M V$ of the maximin design $\xi_{z}^{\text {maximin }}$ as defined in equation (7) is presented in bold face and the second largest minimum relative efficiency is underlined. Table 3 also displays within brackets the mean and standard deviation for the relative efficiency frequency distributions for designs $\xi_{\mathrm{z}}^{\text {maximin }}$, $\xi^{n o r}, \xi^{m i}, \xi^{--u n i}$, and $\xi^{--u n i}$. The same structure applies to Table 4 that displays parallel results 
for CASE 2. The highest minimum relative efficiencies are highlighted in bold, and the second best performance is underlined.

Table 3. Minimum relative efficiencies for the maximin and alternative designs for CASE 1

\begin{tabular}{|c|c|c|c|c|c|}
\hline \multirow[b]{2}{*}{$I_{z}$} & \multicolumn{4}{|c|}{ Designs } & \multirow[b]{2}{*}{$\xi^{\text {sun }}$} \\
\hline & $\xi^{\text {maximin }}$ & $\xi^{\text {nor }}$ & $\xi^{\text {uni }}$ & $\xi^{3 u n i}$ & \\
\hline \multirow[t]{2}{*}{$I_{1}$} & 0.8128 & 0.5258 & $\underline{0.7106}$ & 0.6120 & 0.7048 \\
\hline & $(0.96,0.03)$ & $(0.75,0.06)$ & $(0.86,0.06)$ & $(0.83,0.06)$ & $(0.86,0.07)$ \\
\hline \multirow[t]{2}{*}{$I_{2}$} & 0.7548 & 0.4963 & $\underline{0.7106}$ & 0.5794 & 0.7048 \\
\hline & $(0.92,0.05)$ & $(0.76,0.08)$ & $(0.87,0.06)$ & $(0.84,0.08)$ & $(0.87,0.07)$ \\
\hline \multirow[t]{2}{*}{$I_{3}$} & 0.7226 & 0.4963 & $\underline{0.7106}$ & 0.5794 & 0.7048 \\
\hline & $(0.88,0.06)$ & $(0.76,0.08)$ & $(0.88,0.06)$ & $(0.84,0.08)$ & $(0.86,0.07)$ \\
\hline \multirow[t]{2}{*}{$I_{4}$} & 0.7955 & $\underline{0.6262}$ & 0.5812 & 0.5619 & 0.5447 \\
\hline & $(0.95,0.04)$ & $(0.76,0.05)$ & $(0.80,0.09)$ & $(0.80,0.08)$ & $(0.78,0.11)$ \\
\hline \multirow[t]{2}{*}{$I_{5}$} & 0.7354 & 0.6232 & 0.6573 & $\underline{0.6914}$ & 0.5356 \\
\hline & $(0.90,0.06)$ & $(0.86,0.07)$ & $(0.86,0.08)$ & $(0.90,0.07)$ & $(0.79,0.11)$ \\
\hline \multirow[t]{2}{*}{$I_{6}$} & 0.6967 & 0.5870 & $\underline{0.5991}$ & 0.5844 & 0.5356 \\
\hline & $(0.91,0.06)$ & $(0.80,0.09)$ & $(0.87,0.08)$ & $(0.86,0.09)$ & $(0.84,0.11)$ \\
\hline \multirow[t]{2}{*}{$I_{7}$} & 0.7348 & 0.5108 & $\underline{0.5938}$ & 0.5826 & 0.5525 \\
\hline & $(0.93,0.06)$ & $(0.73,0.07)$ & $(0.83,0.10)$ & $(0.80,0.09)$ & $(0.84,0.12)$ \\
\hline \multirow[t]{2}{*}{$I_{8}$} & 0.6789 & 0.4963 & $\underline{0.6573}$ & 0.5794 & 0.5356 \\
\hline & $(0.87,0.07)$ & $(0.83,0.09)$ & $(0.87,0.07)$ & $(0.88,0.08)$ & $(0.81,0.11)$ \\
\hline \multirow[t]{2}{*}{$I_{9}$} & 0.6792 & 0.4963 & $\underline{0.6573}$ & 0.5794 & 0.5356 \\
\hline & $(0.87,0.08)$ & $(0.80,0.10)$ & $(0.87,0.07)$ & $(0.86,0.08)$ & $(0.83,0.11)$ \\
\hline
\end{tabular}

Note: The mean and standard deviation of the relative efficiencies are given in brackets.

The second best performance in terms of minimum relative efficiency is underlined.

maximin design $\xi_{\mathrm{z}}^{\text {maximin }}$ as defined in equation (7) is presented in bold face and the second largest minimum relative efficiency is underlined. Table 3 also displays within brackets the mean and standard deviation for the relative efficiency frequency distributions for designs $\xi_{\mathrm{z}}^{\operatorname{maximin}}, \xi^{\text {gor }}, \xi^{m i n}, \xi^{- \text {-uni}}$, and $\xi^{\xi^{-u n i}}$. The same structure applies to Table 4 that displays parallel results for CASE 2. The highest minimum relative efficiencies are highlighted in bold, and the second best performance is underlined.

As can be seen in Tables 3 and 4, the maximin efficiency is primarily influenced by composition of the corresponding item space. Furthermore, not only the performance of the maximin design, but also of the alternative designs is affected by this factor. Tables 3 and 4 
show that the performances of the designs in terms of mean efficiency and of $\left[\min _{I}\right.$ eff $\left.(\xi)\right]$ decrease, as the imposed restrictions on the discrimination parameters range

Table 4. Minimum relative efficiencies for the maximin and alternative designs for $C A S E 2$

\begin{tabular}{|c|c|c|c|c|c|}
\hline \multirow[b]{2}{*}{$I_{z}$} & \multicolumn{4}{|c|}{ Designs } & \multirow[b]{2}{*}{$\xi^{\text {5uni }}$} \\
\hline & $\xi^{\text {maximin }}$ & $\xi^{n o r}$ & $\xi^{u n i}$ & $\xi^{3 u n i}$ & \\
\hline \multirow[t]{2}{*}{$I_{1}$} & $\underline{0.6440}$ & 0.4042 & 0.6404 & 0.4489 & 0.6664 \\
\hline & $(0.87,0.07)$ & $(0.66,0.11)$ & $(0.82,0.06)$ & $(0.74,0.12)$ & $(0.85,0.06)$ \\
\hline \multirow[t]{2}{*}{$I_{2}$} & $\underline{0.6375}$ & 0.3626 & 0.6007 & 0.3694 & 0.6452 \\
\hline & $(0.82,0.07)$ & $(0.65,0.12)$ & $(0.81,0.07)$ & $(0.73,0.14)$ & $(0.84,0.06)$ \\
\hline \multirow[t]{2}{*}{$I_{3}$} & 0.5704 & 0.3074 & 0.5288 & 0.2587 & $\underline{0.5635}$ \\
\hline & $(0.83,0.09)$ & $(0.64,0.14)$ & $(0.79,0.08)$ & $(0.70,0.16)$ & $(0.82,0.08)$ \\
\hline \multirow[t]{2}{*}{$I_{4}$} & $\mathbf{0 . 5 8 7 7}$ & 0.4359 & $\underline{0.5479}$ & 0.4699 & 0.5420 \\
\hline & $(0.84,0.10)$ & $(0.69,0.10)$ & $(0.78,0.08)$ & $(0.74,0.11)$ & $(0.78,0.10)$ \\
\hline \multirow[t]{2}{*}{$I_{5}$} & $\underline{0.5100}$ & 0.3331 & $\mathbf{0 . 5 7 3 2}$ & 0.2533 & 0.4884 \\
\hline & $(0.78,0.11)$ & $(0.70,0.15)$ & $(0.78,0.08)$ & $(0.74,0.17)$ & $(0.77,0.12)$ \\
\hline \multirow[t]{2}{*}{$I_{6}$} & $\underline{0.5229}$ & 0.3402 & 0.5631 & 0.2581 & 0.4940 \\
\hline & $(0.80,0.11)$ & $(0.67,0.13)$ & $(0.80,0.09)$ & $(0.73,0.15)$ & $(0.82,0.11)$ \\
\hline \multirow[t]{2}{*}{$I_{7}$} & 0.5149 & 0.4054 & 0.5586 & 0.4405 & $\underline{0.5476}$ \\
\hline & $(0.82,0.11)$ & $(0.65,0.11)$ & $(0.79,0.09)$ & $(0.71,0.12)$ & $(0.82,0.10)$ \\
\hline \multirow[t]{2}{*}{$I_{8}$} & 0.4474 & 0.3084 & 0.5441 & 0.2383 & $\underline{0.4884}$ \\
\hline & $(0.75,0.12)$ & $(0.67,0.16)$ & $(0.77,0.09)$ & $(0.71,0.17)$ & $(0.78,0.11)$ \\
\hline \multirow[t]{2}{*}{$I_{9}$} & 0.4594 & 0.2872 & 0.5155 & 0.2387 & $\underline{0.4940}$ \\
\hline & $(0.77,0.13)$ & $(0.64,0.16)$ & $(0.78,0.17)$ & $(0.69,0.17)$ & $(0.79,0.12)$ \\
\hline
\end{tabular}

Note: The mean and standard deviation of the relative efficiencies are given in brackets. The second best performance in terms of minimum relative efficiency is underlined.

and combinations from $I_{1}$ through $I_{9}$ are relaxed. The lowering effect on relative efficiencies caused by the enlarged range of the location parameters values becomes also evident by comparing Table 3 (CASE 1) with 4 (CASE 2).

The design performances of item spaces from the first row (Table 1) are in general higher than their counterparts of the second and third rows, following the enlargement of the parameter ranges. Analogously, a switch from the row- to a column-perspective also enables a comparative assessment of the designs' efficiencies. For the item spaces in the first column, i.e. $I_{1}, I_{4}$ and $I_{7}$, an extra imposition forced the parameters of the last two adjacent categories to lie relatively close to each other with $d_{\max }=0.5$. As a result, their constituent items were 
more alike, which reflected on an increased maximin relative efficiency, the highest of them 0.8128 , for CASE 1 , and 0.6440 for CASE 2 .

By contrast, for item space $I_{9}$, which allowed for a more varied combination of discrimination parameters, i.e. low/low, low/high and high/high for fixed pairs of $a_{i 2}$ and $a_{i 3}$, the relative efficiencies of its maximin designs was lowered. Note, also, that with an increase of variation of parameters values for CASE 1, the probability mass functions became more symmetric (Figure 2).

Striking is the finding that, by considering larger range of values for difficulty parameters (CASE 1 versus $C A S E 2$ ), the maximin D-optimal design looses its leading position in the comparative evaluation of performances.

The general configuration of the maximin designs, represented by the location of the support points and their respective weights (Figure 2 and 3), deserves some final remarks.

It has been previously mentioned that a maximin design is expected to be symmetric and balanced (Ouwens et al., 2002), under strict conditions of a compactness and symmetry of parameters space. Here, however, focus has been primarily given to D-optimal designs of a finite set of items. As a consequence, the configurations of the maximin designs were adjusted to the composition of their respective item spaces. Nevertheless, a tendency towards symmetry could be detected, once the parameter combinations were allowed to vary more freely (see for instance the maximin designs for $I_{8}$ and $I_{9}$ for CASE 1), or alternatively, once a greater discriminatory distance between the categories, i.e. $d_{\min }=0.71$, was possible.

It should be reminded that, for polytomous items, the shape of the item categories characteristic curves (ICCCs) is not primarily determined by the discrimination parameter, but rather by how discrimination and location parameters interact. Running the maximin algorithm with an enlarged location parameters range, as given by $C A S E 2$, poses a little inconvenience in terms of the fixed combinations of parameters values. By allowing arbitrary combinations to be made out of the increased range, the chance of having items characterised by rather implausible ICCCs, i.e. non-discriminatory lines along the $\theta$ scale, is considerably enhanced. See for instance Figure 4(a) and 4(b), where the ICCCs of items characterised by the different parameters are displayed. The example displayed in 4(a) illustrates how this item would be of little use in their main task of discriminating among neighbouring $\theta$ s once compared to the items shown in $4(\mathrm{~b})$.

The likelihood of having such 'deviant' items in the pool is increased trough an enlargement of the $c_{i g}$ range. The low discrimination of deviant items in the pool possibly explains why the maximin designs' support points are conspicuously closer and more 
4(a). Item 1

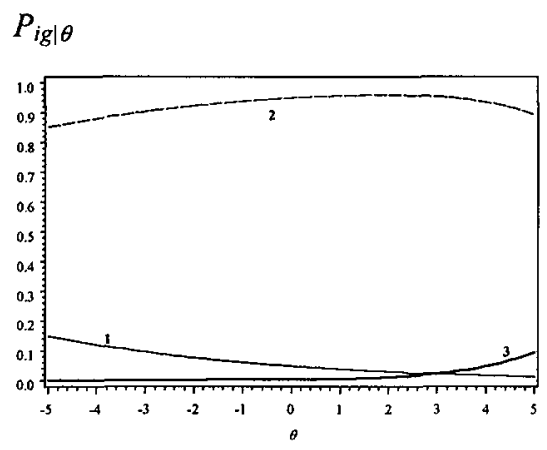

$a_{i g}=[0,0.25,1]$

$c_{i g}=[0,3,-3]$ 4(b). Item 2

$P_{i g \mid \theta}$

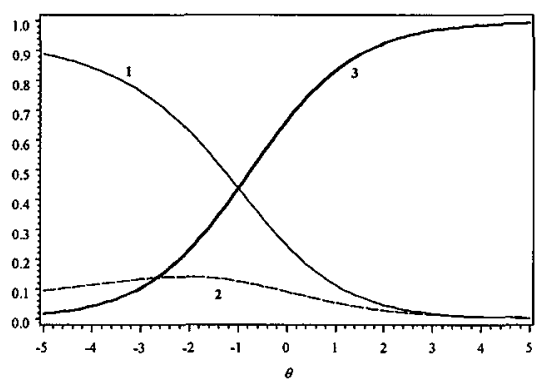

$a_{i g}=[0,0.25,1]$

$c_{\text {ig }}=[0,-1,1]$

Figure 4. The item categories characteristic curves (ICCCs) for two three-categories NRM items and their parameters .

heavily represented at the ends of the $\theta$ range for CASE 2 (see Figure 3). This leads to a final topic of discussion, namely a short comparison between the dichotomous and the polytomous calibration designs.

In calibration of dichotomous items it is well known that lower discrimination parameters push the support points towards the extremities of the $\theta$ scale (Stocking, 1990). The same shift occurs for polytomous items. See probability mass functions (pmfs) of $I_{l}$ and $I_{2}$ in Figures 2 and 3. A further manifestation of this 'low-discrimination' effect can also be seen for item spaces, which allowed a $d_{\min }=0.15$. Here the support point on the right side of the pmfs is pushed to the right extreme of the latent trait scale $\left(I_{1}, I_{4}\right.$ and $\left.I_{7}\right)$.

Such result is intuitively easily grasped. Flat probability response curves mean that an item cannot distinguish well among the trait levels along the $\theta$-scale, let alone among close neighbouring traits. Hence, estimating the slope coefficient (i.e. the discrimination parameter) of such curve is a task better accomplished by traits, whose probabilities of response differ the most, i.e. the farther the better. Thus, extreme values of $\theta$ give more information on $a_{i g}$ 's for estimable categories, whose characteristic curves cannot be clearly held apart from each other. 


\subsection{Discussion}

The purpose of finding optimal calibration designs for item calibration is to save time and money. An optimal calibration design could reduce the number of required examinees considerably, while the efficiency of the item parameter estimates is kept as large as possible. The calibration of items, however, is often hampered by the fact that optimal calibration designs cannot be found without knowing the exact values of the item parameters. Optimal designs can only be found locally, i.e. for a certain set of item parameters. As such, local optimality represents only a spurious solution to the problem of Fisher's Information parameters dependence in IRT models.

In this paper this problem has been dealt with by introducing a maximin procedure. This takes into account the fact that exact item parameter values are not known in advance. Rather, maximin designs are based on pre-specified ranges of item parameters. Since these designs are defined by means of the minimum efficiency, their performance is always measured by the worst possible situation, i.e. by the minimum relative efficiency measure.

The performance of maximin designs for polytomously scored items has been evaluated and compared to some well-known calibration designs. The evaluation was based, for each case, on nine different item spaces, which were considered to represent a wide range of item parameter values found in practice.

The evaluated item spaces were mainly characterized by the variation among the discrimination parameters $a_{i g}$. This variation, in turn, was controlled over the width of the parameters range as well as over the degree of diversity among parameters combinations. The range of difficulty parameters, on the other hand, was fixed at $[-1,1]$ for CASE 1 , and $[-3,3]$ for $C A S E 2$.

It has been shown that the performance of maximin designs decreases as the overall variation of the discrimination parameter values increases. The same applies to the alternative designs. Among these, the uniform design $\xi^{i n i}$ has come out as rather consistent. In spite of the fact, that its minimum efficiency was negatively affected by an increased parameters variation, its sensitivity to the latter seemed to be less accentuated, contrary to the other alternative designs. Similar results to the ones here presented, can be replicated with any other specifications of designs and/or parameter spaces, with the general tendency that the more varied the composition of the item space, the greater the loss of the designs' efficiency. 
Some remarks on the practical consequences of this evaluation should be made. In general the maximin D-optimal design performs best for CASE 1, where the location parameters range is smaller. Since these maximin designs are D-optimal designs, they are based on at most three distinct support points on the latent trait scale for the three categories NRM. In some practical cases, however, it may not be possible to select examinees having just three distinct latent trait values. In that case, the uniform calibration design will be a good alternative. We have seen that, for CASE 1, the performances of maximin and uniform designs are rather comparable, once the imposed restrictions on ranges and parameter combinations are reduced. In CASE 2, the performance of the more uniform designs manages to surpass the one of the maximin D-optimal designs. These results tend to favour, at first, a calibration sample with latent traits uniformly distributed. This is, furthermore, reinforced by another aspect of the practical reality, namely the general lack of knowledge about the prior distribution of parameters values. On these premises, it is reasonably safe to recommend the use of a uniform design to calibrate polytomously scored items described by the NRM, for this design seems to be rather robust against deviant parameter combinations. Following this same path of logic, the use of the normal sampling design is less recommendable. This is to say that the method that is often applied in practice, i.e. a random sample of examinees, whose traits are normally distributed, can be rather inefficient for the job. This reservation can be, however, lifted, in case some prior information about the parameters distribution is available. If this prior information resembles to a certain extent the conditions represented by, for instance, the item space $I_{4}$ in $C A S E 1$, then the application of the normal sampling design would not incur in great loss of estimates precision. At any rate, this conjecture demonstrates that, even if not easily attainable in practice with regards to the specific support points required, a maximin design can always be used as a quantitative criterion, upon which design performances can be compared. It serves as a reference to help in the selection of possible calibration designs.

Furthermore, the results here presented concern primarily items described by the three categories NRM. Although not carried out in this work, it is assumed that the findings will not drastically change for items with more than three categories. The following can be expected: It is known in advance that more categories would add extra support points of the local D-optimal designs, since the maximum number of support points is equal at most to the number of estimable discrimination parameters plus one $\left(p^{*}+1\right)$. An increase in number of support points means, at the same time, that the designs become overall less discrepant among themselves, which, in turn, is bound to result in an increase of their relative 
efficiencies. It can be expected that an increase of the number of support points will also bring prominence to the uniform design as an efficient design.

At last, nothing hinders the expansion and application of the same maximin rationale to other IRT polytomous models. It remains, however, to be seen, whether the comparative performance evaluation with alternative designs would yield similar results, which tended to favour uniform over other non-optimal alternative designs. 


\section{Appendix . The D-optimal Maximin Algorithm}

The description is displayed in two parts: the premises required to initiate the algorithm and an outline of its structure.

\section{Premises:}

Select an item space $I$ and its corresponding D-optimal design space $\mathrm{X}^{*} \subset \mathrm{X}$, where $\xi_{j}^{*} \in \mathrm{X}^{*}$ are the locally D-optimal designs of the items $i, j \in I$, with $i, j=1, \ldots, n$. The D-optimal designs can be obtained sequentially, in a procedure that has been described by Berger (1992) or Holman and Berger (2001).

\section{Algorithm:}

Step 1: Apply a D-optimal design $\xi_{j}{ }^{*} \in \mathrm{X}^{*}$ to an item $i \in I$ and compute the

$$
\operatorname{Det}\left[\operatorname{Inf} f_{i}\left(\xi_{j}^{*}, a_{i}, c_{i}\right)\right] \text {; }
$$

Step 2: $\quad$ Compute the relative efficiency $\operatorname{eff}\left(\xi_{j}^{*}\right)$, given by equation (6);

Step 3: $\quad$ Repeat step 1 and 2 until the design $\xi_{j}^{*}$ has been applied to all $n$ items of item space $I$. At the end of step 3, a set of $n$ relative efficiencies $\operatorname{eff}\left(\xi_{j}^{*}\right)$ is available.

Step 4: Select the smallest value $\left[\min _{I} \operatorname{eff}\left(\xi_{j}^{*}\right)\right]$ from the set of relative efficiencies;

Step 5: $\quad$ Return to Step 1 with a new design measure $\xi_{j+1}^{*} \in \mathrm{X}^{*}$, set $\xi_{j}^{*}=\xi_{j+1}^{*}$ and

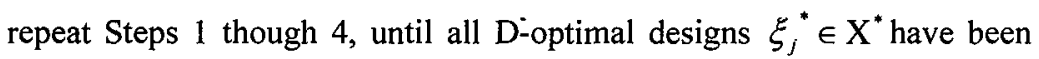
taken into account. After the completion of Step 5, a set of minimum relative efficiencies $\left[\min _{I} \operatorname{eff}\left(\xi_{j}^{*}\right)\right]$ over the space $\mathrm{X}^{*}$ is available. Each of these minimum values is associated with one design $\xi_{j}^{*} \in \mathrm{X}^{*}$;

Step 6: $\quad$ Select the maximin design $\xi^{\text {maximin }} \in \mathrm{X}^{*}$ linked to the highest minimum relative efficiency, so that: $M M V=\max _{\mathrm{x}^{*}}\left[\min _{I} e f f\left(\xi_{j}^{*}\right)\right]$ 


\section{Test design optimisation for the Nominal Response Model}

This chapter has been submitted for publication with co-authors M.P.F. Berger, A.M.M. Muijtjens and F.E. S. Tan.

Acknowledgments: We wish to thank Arno Muijtjens for calibrating and putting item parameters from the progress test at our disposal. 


\title{
Test design optimisation for the Nominal Response Model
}

\begin{abstract}
The problem of optimal test assembly for items described by polytomous IRT models has been hitherto little explored. In this paper, statistical techniques of design optimisation are applied to assemble a test composed of items described by the Nominal Response Model (NRM). The items stem from the so-called progress test, annually administered to medical students at the University of Maastricht, the Netherlands. For the selection of items for a fixed-form, group-based test, an algorithm based on two distinct (A- and D-) optimality criteria is applied. Both criteria are objective functions of Fisher's Information and their different selective mechanisms are closely investigated. Further variable factors, whose influence on the final selection is scrutinised, are the item pools composition and the distribution of the latent trait variables representing the groups of examinees to be tested. This study shows how these factors interact to determine the final test composition. A better understanding of the optimisation modus operandi enables practitioners to actively adapt the items selection to the specific objectives and demands of the test.
\end{abstract}

Keywords: Optimal Test Design, Polytomous IRT Model, Optimality Criteria, Fisher's Information 


\section{Test design optimisation for the Nominal Response Model}

\subsection{Introduction}

Modern psychological assessment is characterised by an ever-growing sophistication of measurement methods targeting quantification of psychological and educational traits. In this endeavour, application of statistical models has been rather helpful, and several optimisation techniques have been developed to maximise the usefulness of the models (van der Linden, 1998). Most of these techniques are brought together under the shorthand term of Optimal Test Assembly.

Applied Psychological Measurement (APM) dedicated a special issue (1998) to modern developments in this field, in which several practical and theoretical problems are handled. In terms of Item Response Theory (IRT) models, the overwhelming majority of the papers focused on unidimensional dichotomous models. More recently, Veldkamp (2001) investigated the techniques of optimal test assembly for multidimensional models, albeit still concentrating on binary response patterns.

Van der Linden (1998) describes four basic approaches to find a solution to an optimal tests assembly problem. He divides them into the heuristic-based test assembly (Luecht, 1998 and Sanders and Verschoor, 1998), the 0-1 linear programming (van der Linden and Reese, 1998), the network-flow programming (Armstrong, Jones and Kunce (1998) and eventually the optimal design approach (Berger, 1998).

The latter approach makes use of statistical techniques of design optimisation in order to select the best design points envisaging efficient estimation of the model parameters. Considering the fact that IRT models are usually characterised by both items and examinee's parameters, there are correspondingly two cases of design optimisation. On the one hand, the design points (the trait levels and their corresponding weights) are taken from the latent trait range, in order to enhance the precision of items parameters estimators. This case is known as optimal sampling or calibration design. Optimal test design, on the other hand, refers to the search of the most suitable items in terms of their parametric characteristics (the design points) to provide an accurate estimation of the latent trait, $\theta$. For a general overview of optimal design in educational testing see van der Linden (1994) and Buyske (2003). The present work focuses on optimal test design. For a detailed review on the optimality criteria used in an IRT context see Berger and Veerkamp (1996). 
Test design optimisation has been previously carried out for dichotomous IRT models with a few optimality criteria, among them the A- and D-optimality criteria, (Berger and van der Linden, 1995). Differences in the final test composition between these two criteria have been reported but the reasons behind them not fully elucidated. So far, one paper has specifically targeted test design optimisation for polytomous models (Berger and Mathijssen, 1997). In this same line of research, the current study applies an optimisation technique for the assembly of items described by the Nominal Response Model (NRM). The items selection will be based on the A- and D-optimality criteria. The objective is to investigate and explain how their different selective mechanisms determine the final test composition, under varied conditions of item pool information and sample of test-takers. The perspective of attaining a variety of 'optimal assemblies' by different criteria is relevant for the practice. It opens the possibility of suitably matching the item selection procedure to specific test goals and requirements.

Optimising test designs often means selecting the most informative items to constitute the test for the purpose of trait estimation. Herein, the question of items eligibility for test assembly will be restricted to their parametric characteristics.

The whole optimisation process will be based on real parameter values of items from the so-called progress test, administered in the faculty of Medicine at the University of Maastricht, the Netherlands.

\subsection{The Nominal Response Model (NRM)}

\subsubsection{The Nominal Response Model}

The NRM was firstly introduced by Bock (1972). The NRM is considered the most general model in the divide-by-total model classification category (Thissen and Steinberg, 1986). It does not require the ordering of categories to reflect different degrees of the trait level to be measured. It is, therefore, suitable for nominal data (Heinen, 1996, p.103). The equation that describes the NRM is:

$$
P_{i g \mid \theta_{t}}=\frac{\exp \left(c_{i g}+a_{i g} \theta_{t}\right)}{\sum_{h=I}^{k_{i}} \exp \left(c_{i h}+a_{i h} \theta_{l}\right)},
$$


where $a_{i g}$ is the discrimination parameter of category $g$ of item $i, i=1, \ldots, n, c_{i g}$ is the location parameter associated with category $g$ of item $i, k_{i}$ is the number of categories of item $i$ with $(g, h)=1,2, \ldots, k_{i}, \theta_{l}$ is the latent trait variable of examinee $t$, with $t=1, \ldots, T$, and $P_{i g \mid \theta_{t}}$ is the probability of examinee with trait $\theta_{l}$ of choosing category $g$ of item $i$.

The part of the $\log$ likelihood function, $\log L$ that needs to be maximised in order to obtain the maximum likelihood (ML) estimates is given by:

$$
\sum_{i} \sum_{i} \sum_{g} f_{i t g} \log P_{i g \theta_{i}}
$$

where $f_{\text {itg }}$ is the number of examinees with ability $\theta_{t}$ that respond to each of the $k_{i}$ categories of item $i$. The probability $P_{i g \mid \theta_{t}}$ is defined in equation (1). The Newton-Raphson procedure is commonly used to obtain the ML estimates of the latent trait. This procedure requires the first and second derivatives of $\log L$ with respect to the trait $\theta_{t}$ (see Baker, 1992, p.268-272, among others).

For the maximum likelihood estimation of the item parameters, the first and second derivatives of log-likelihood function are taken with respect to the item parameters. For this purpose, certain constraints must be imposed on the values of discrimination and location parameters, in order to avoid problems of identification. In this paper, the constraint will be used that sets $a_{i 1}=c_{i 1}=0$, for all $i$, and the categories will be ordered according to their discrimination values, i.e. $a_{i 1}<a_{i 2} \ldots<a_{i g}$. See Heinen (1996, p.102), for a discussion on these constraints.

\subsubsection{Fisher' Information for the NRM}

Within an IRT framework, optimal test design envisages improvement of the efficiency of the latent trait estimator, $\hat{\theta}$. Test design optimisation deals with the process of selecting 'best' items from an available pool for the purpose of test construction. The criterion of selection is often based on Fisher's information, or an objective function of the latter. An increase of this information will eventually improve efficiency of the latent trait estimator, $\hat{\theta}$. Fisher's information for the NRM of an item $i$ on a single $\theta_{t}$ (Heinen, 1996) is given by: 


$$
\operatorname{Inf}_{t, \theta_{t}}=\left\{\sum_{g} a_{i g}^{2} P_{i g \mid \theta_{t}}-\left[\sum_{g} a_{i g} P_{i g \mid \theta_{t}}\right]^{2}\right\} .
$$

The index $g$ runs through the number of categories $k_{i}$ of item $i$, i.e. $g=1,2, \ldots, k_{i}$ with $a_{i g}$ standing for their corresponding discrimination parameters. $P_{i g \mid \theta_{t}}$ is given by equation (1).

\subsection{Test design optimisation}

The optimisation procedure described in this paper is applicable to a group-based test setting. This means that test items will be selected in order to maximise Fisher's Information over a group of examinees, each represented by his/her trait level $\theta_{l}$. In practice, such setting corresponds to a paper and pencil ( $p \& p$ ) test situation, where all students respond to the same items at the same time. Their traits estimation takes place simultaneously. Such group-based test stands in contradistinction to the individually tailored tests, where items are administered in succession to a single student, and the selection of an item is adjusted to the sequentially updated trait estimator. The latter case, involving an individual $\theta$-estimation, reflects the modern computerised adaptive testing (CAT) and will not be considered within the scope of this paper.

An optimal test design procedure has been implemented to select a fixed number of $n$ items representing the test length with $i=1,2, \ldots, n$. The $n$ items will be selected from a large item bank $I$, containing in total $N$ items with known parameters, where $n<<N$.

The constituent items $i \in I$ are multi-categorical items described by the Nominal Response Model (NRM), whose item parameters are described by a vector of discrimination parameters, $a_{i}=\left[a_{i 1}, \ldots, a_{i g}, \ldots, a_{i k_{i}}\right]$ and a vector of location parameters, $c_{i}=\left[c_{i 1}, \ldots, c_{i g}, \ldots, c_{i k_{t}}\right]$ with $g=1,2, \ldots, k_{i}$, the number of item's categories. The test design space is the item pool, from whence optimal design points, i.e. $n$ items are chosen. The latent trait vector $\Theta$ entails the test-takers trait levels, i.e. $\Theta=\left[\theta_{1}, \ldots, \theta_{l}, \ldots, \theta_{T}\right], t=1, \ldots, T$, with $T$ representing the total number of examinees in the group. Fisher's information of $n$ items on all traits $\Theta$ can be gathered on a vector $M\left(\Theta \mid a_{i}, c_{i}, i=1,2, \ldots, n\right)$ with:

$$
\boldsymbol{M}\left(\Theta \mid a_{i}, c_{i}, i=1,2, \ldots, n\right)=\left[\operatorname{Inf} f_{\theta_{1}}, \operatorname{Inf}_{\theta_{2}}, \operatorname{Inf}_{\theta_{3}}, \ldots, \operatorname{Inf} f_{\theta_{r}}\right]
$$


and $\operatorname{Inf} f_{\theta_{t}}=\sum_{i} \operatorname{Inf} f_{i, \theta_{t}}$.

By considering group-based or simultaneous optimisation, different optimality criteria can be taken into account as the objective function of Fisher's Information $\Phi\left\{\left(M\left(\Theta \mid a_{i}, c_{i}, i=1,2, \ldots, n\right)\right\}\right.$. Irrespective of the underlying objective function, the search of an optimal test assembly with $n$ items can be formulated as a mathematical programming problem:

$$
\text { Maximise } \Phi\left\{\left(M\left(\Theta \mid a_{i}, c_{i}, i=1,2, \ldots, n\right)\right\}\right.
$$

constrained by a chosen test length $n$. The maximisation on (5) can be subject to further restrictions imposed either on the vector of latent traits $\Theta$, i.e. its size and the frequency distribution of $\theta_{t} \in \Theta$, or on the set of item parameters comprising the $n\left\{a_{i}, c_{i}\right\}$. The latter constraints often relate to issues of practical relevance as content and item exposure specifications, usually having a restrictive effect on item selection. For the present purpose of comparing and clarifying fundamental differences of the criteria, these restrictions will be disregarded. The selection of items will be carried out exclusively on the grounds of their statistical properties, which are dependent on the items' known parameter values.

An algorithm that goes through all possible $n$ items' combinations in the search for a optimal solution for the maximisation problem in (5) can be rather time consuming. A quicker alternative is the sequential search, i.e. one item at a time. The sequential procedure was firstly introduced by Wynn (1970), modified by Berger (1994a, b) for test designs and shown by Berger (1992) and Holman and Berger (2001) to be also feasible for optimal calibration designs. In all cases, the sequential solution has been shown to offer a good approximation to the optimal solution.

The sequential procedure for fixed-form test design can be summarised as follows: Select from the available pool $I$ an item $i \in I$, for which $\Phi\left\{M\left(\Theta \mid a_{i}, c_{i}\right)\right\}$ is maximised, until a pre-determined number of items $n$ has been selected to compose the optimal test. Contrary to (4), $M\left(\Theta \mid a_{i}, c_{i}\right)$ is a vector containing Fisher's Information of a single item $i$ on all traits $\theta_{l} \in \Theta$, i.e. $M\left(\Theta \mid a_{i}, c_{i}\right)=\left[\operatorname{Inf} f_{i, \theta_{1}}, \operatorname{Inf} f_{i, \theta_{2}}, \operatorname{Inf} f_{i, \theta_{3}}, \ldots, \operatorname{Inf} f_{i, \theta_{T}}\right]$. The sequential algorithm is described in detail in appendix 1 , which can be easily adjusted to accommodate practical constraints. 


\subsubsection{Optimality criteria}

In a more general setting of design optimisation, Atkinson and Donev (1996) provide an overview of diverse optimality criteria $\Phi$. A closer description of optimality criteria characteristics in the specific context of optimal test design is given by Berger and Veerkamp (1996). The present investigation concentrates on two objective functions of Fisher's Information, known as the A- and D-optimality criteria.

The A-optimality, trace or sum criterion, is defined as:

$$
\Phi_{\mathrm{A}}\left\{\left(\boldsymbol{M}\left(\Theta \mid a_{i}, c_{i}, i=1,2, \ldots, n\right)\right\}=\sum_{i=1}^{T} \operatorname{Inf}_{\theta_{t}}\right.
$$

A frequently applied criterion is known as the product or determinant or D-optimality criterion:

$$
\Phi_{\mathrm{D}}\left\{\left(M\left(\Theta \mid a_{i}, c_{i}, i=1,2, \ldots, n\right)\right\}=\prod_{i=1}^{T} \operatorname{Inf} f_{i}\right.
$$

which can be alternatively written as $\Phi_{\mathrm{D}}\left\{\left(M\left(\Theta \mid a_{i}, c_{i}, i=1,2, \ldots, n\right)\right\}=\sum_{t=1}^{T} \log \operatorname{Inf} f_{\theta_{t}}\right.$.

The reason for choosing these two criteria is that, given the premises of a group-based test, they can be regarded as a summary measure, conjoining information values along the $\theta$ range of interest. The A- and D- optimality criteria envisage the maximisation of $\Phi\left\{\left(M\left(\Theta \mid a_{i}\right.\right.\right.$, $\left.\left.c_{i}, i=1,2, \ldots, n\right)\right\}$ over the vector $\Theta$, either by the summation or product of the $\ln f_{\theta_{1}}$ values (equations 6 and 7).

The A-optimality criterion is a standard criterion in literature of test design. Given equally weighted traits, it can be interpreted as the area under the curve of the test information function $I N F$, computed as a sum of individual item information functions: $I N F=\sum_{i=1}^{n} I n f_{i}$. The latter, i.e. $I n f_{i}$ is the information function of an item along the trait range.

The D-criterion, though not often associated with test assembly, is known to offer a few advantages over other criteria. It is inversely proportional to the volume of confidence ellipsoid of the parameters estimators and it has been shown to be invariant under linear transformation of the logit scale. In the specific domain test design optimisation, this means 
that a D-optimal test design would remain the same, irrespective of the scale used to measure the latent trait variable. Additionally, Berger and van der Linden (1995) have reported for the dichotomous models that the test assembly based on the $\mathrm{D}$-criterion can conditional on the latent trait distribution significantly diverge from the assembly of other criteria. This is a major argument for its inclusion in this study. Understanding how different criteria modus operandi can affect the final assembly is a first step towards matching the item selection procedure to the final test's objective.

\subsection{A numerical study}

\subsubsection{Progress test}

The Faculty of Medicine, at the University of Maastricht (UM) has been making use of 'large scale' tests to assess students' knowledge growth along their graduate years in a problem-based learning environment (van der Vleuten et al., 1996). These tests are administered four times a year, with the objective to measure the progress of students of all grades at the UM towards the end objectives of the graduation program. They are referred to as 'progress tests'. Detail description of the philosophy, rationale and utility of progress tests have been described by Imbos et al. (1990), Tan et al. (1995) and van der Vleuten et al. (1996).

\subsubsection{Selection of items}

The item parameters used to compose the item banks are taken from the progress test. These items have three nominal categories (additionally to correct/incorrect, there is also the 'don't know' -DK- response option). The NRM has been selected for the task of calibrating the items of progress test with $k_{i}=3$ categories.

In setting progress tests into an IRT context, one has to bear in mind that these tests are specifically made to cover the broad domain of medical expertise. The breadth of disciplines might cause the trait $\theta$ to be more a multi-, instead of a unidimensional construct. Yet, since unidimensionality of the latent trait is an important assumption for the NRM, it has been decided to pre-select items in such a way as to safeguard it. Factor analysis has been applied to single out the discipline that manifested a greater unidimensionality of items scores: internal medicine. Further factorisation selected 17 out of 37 available internal 
Table 1. Estimated parameters of the $n=17$ calibrated three categories NRM items $(g=3)$ from internal medicine with their descriptive statistics and correlation matrix

\begin{tabular}{llrlllr}
\hline$i$ & $a_{i 1}$ & $a_{i 2}$ & $a_{i 3}$ & $c_{i 1}$ & $c_{i 2}$ & $c_{i 3}$ \\
& & & & & & \\
\hline 1 & 0 & 1.065 & 0.920 & 0 & -2.460 & -1.555 \\
2 & 0 & 1.875 & 1.98 & 0 & -1.230 & 1.060 \\
3 & 0 & 1.575 & 1.925 & 0 & -4.085 & -3.520 \\
4 & 0 & 1.355 & 1.650 & 0 & -0.885 & 0.620 \\
5 & 0 & 1.775 & 1.885 & 0 & -1.745 & -0.810 \\
6 & 0 & 1.730 & 1.665 & 0 & -2.610 & -1.420 \\
7 & 0 & 1.410 & 1.780 & 0 & -0.820 & -0.665 \\
8 & 0 & 2.030 & 2.155 & 0 & -1.415 & 0.915 \\
9 & 0 & 3.345 & 3.180 & 0 & -3.965 & -1.940 \\
10 & 0 & 3.015 & 2.425 & 0 & -4.265 & -0.345 \\
11 & 0 & 1.450 & 1.870 & 0 & -2.215 & -1.095 \\
12 & 0 & 2.015 & 2.270 & 0 & -2.665 & 0.075 \\
13 & 0 & 2.515 & 2.150 & 0 & -5.025 & -2.180 \\
14 & 0 & 2.315 & 2.355 & 0 & -3.325 & -2.415 \\
15 & 0 & 1.160 & 2.065 & 0 & -1.895 & 0.075 \\
16 & 0 & 1.765 & 1.600 & 0 & -3.640 & -1.225 \\
17 & 0 & 1.435 & 2.190 & 0 & -2.020 & -1.760 \\
& & & & & & \\
$\boldsymbol{\mu}$ & 0 & 1.874 & 2.004 & 0 & -2.603 & -0.952 \\
$\boldsymbol{\sigma}$ & 0 & 0.625 & 0.468 & 0 & 1.261 & 1.253 \\
& & & & & & \\
\hline
\end{tabular}

Correlation matrix (CORR)

\begin{tabular}{llcrc}
\hline$a_{i 2}$ & & $a_{i 3}$ & $c_{i 2}$ & \multicolumn{2}{c}{$c_{i 3}$} \\
& & & & \\
\hline$a_{i 2}$ & 1.000 & 0.798 & -0.623 & -0.134 \\
$a_{i 3}$ & 0.798 & 1.000 & -0.359 & -0.070 \\
$c_{i 2}$ & -0.623 & -0.359 & 1.000 & 0.665 \\
$c_{i 3}$ & -0.134 & -0.070 & 0.665 & 1.000 \\
\hline
\end{tabular}

medicine items. These 17 items were calibrated by fitting the NRM with the software program MULTILOG (Thissen, 1991).

The parameters of calibrated items are given in Table 1, together with their descriptive statistics and correlation structure. The resultant items' Information Functions $($ Infi) are plotted in Figure 1(a). 


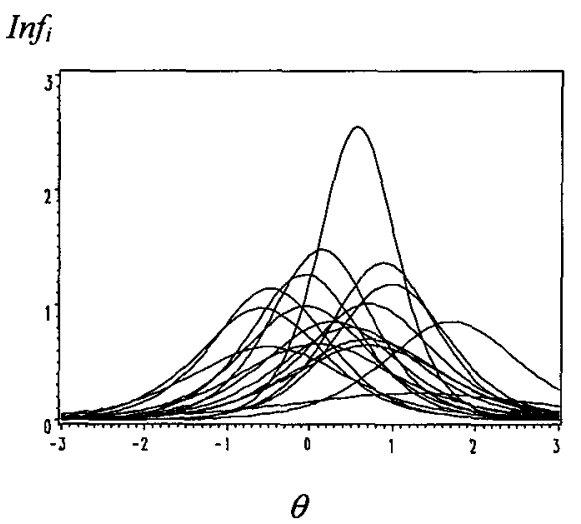

Figure 1(a). Item information functions of the blue print NRM items.

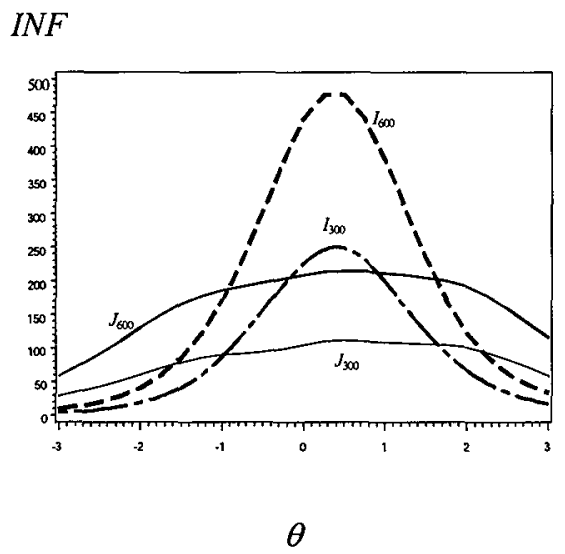

Figure 1(b). Item bank information for four item pools $I_{300}, J_{300}, I_{600}, J_{600}$

\subsubsection{Optimal test designs}

The application of the optimisation algorithm requires a few specifications to be made in advance regarding the item bank $I$, a vector of traits $\Theta$ representing the sample of students, a pre-specified test length, $n$, and an optimality criterion $\Phi($.$) .$

Test length. This comprised 17 items $(n=17)$, in order to equalise the same amount of items calibrated via the NRM. The selection of this test length has been made envisaging a straightforward comparison between the amount of information provided by the original items, on the one hand, and the selected items via optimisation, on the other. The test length, however, could be arbitrarily set to any value. Given a sufficiently large item bank, the test 
length does not affect directly the criteria selective mechanism for the fixed-form test, and as such the final test composition.

The latent trait vector. $T=300$ latent trait values $\theta_{t} \in \Theta$, have been randomly generated either by the standard normal or the uniform distribution, i.e. $\theta_{1} \sim N(0,1)$ and $\theta_{1} \sim U(-3,3)$, producing $\Theta_{N}$ and $\Theta_{U}$, respectively. These two vectors represent groups of examinees, whose trait levels are found, in their overwhelming majority, within the interval [-3,3]. Thus, $\Theta_{N}$ and $\Theta_{U}$ do not differ substantially in their $\theta$-range, but rather in the form of distribution. For efficiency comparison, sub-ranges of the students samples have been also made by partitioning both $\Theta_{N}$ and $\Theta_{U}$ in low, middle and high $\theta$-ranges, with $\theta_{l} \in[-3,-1], \theta_{l} \in[-1,1]$ and $\theta_{t} \in[1,3]$, respectively. These intervals represent more homogeneous groups of examinees as regards their latent trait values.

The item pool composition I. Four item pools, two with $N=300 / 600$ were generated based on the parameters of the 17 calibrated items (Table 1). However, only the results for $N=300$ are presented. The findings for $N=600$ are significantly overlapping with the former and are, therefore, omitted. For parameters generation two cases need to be differentiated.

Firstly, the 17 items have been taken as a blueprint and their parameters have been solely replicated in numbers via random generation, so that an item pool with 300/600 items could be obtained $(N=300 / 600)$. These item pools are referred to as 'peaked', $I$, as opposed to the 'flat' pools, $J$, reflecting the shape of their respective item bank information curves (Figure 1(b)). The flat pool comprises items, whose generation, based on the blue print, has been specifically manipulated to broaden the spectrum of the $\theta$-range covered by their information functions. In either case, the item pools have been obtained through the multivariate normal and multivariate uniform generation of item parameter values $\left(a_{i 2}, a_{i 3}, c_{i 2}\right.$, $\left.c_{i 3}\right)$. Accordingly, the resultant pools were denoted $I_{N}$ and $I_{U}$, for the peaked and $J_{N}$ and $J_{U}$ for the flat pools. It is important to emphasise that for items generation both the mean vector of the original parameter values and their correlation structure, CORR, (Table 1) needed to be incorporated by the normal and uniform probability distributions. This incorporation has been achieved via a SAS macro (SAS Institute Inc., version 8.2) that uses Cholesky decomposition of the variance-covariance matrix (COV) for this purpose. Hence, in the case of $I_{N}$, the generated vector of item parameter values $\left[a_{i 2}, a_{i 3}, c_{i 2}, c_{i 3}\right]^{\prime}$ is normally distributed with a mean vector $\boldsymbol{\mu}$ and variance-covariance matrix $\boldsymbol{\Sigma}$, i.e. $\left[a_{i 2}, a_{i 3}, c_{i 2}, c_{i 3}\right]^{\prime} \sim N(\boldsymbol{\mu}, \boldsymbol{\Sigma})$. For $I_{U},\left[a_{i 2}, a_{i 3}, c_{i 2}, c_{i 3}\right]^{\prime} \sim U(\mu, \Sigma)$. For the flat pools, however, the picture is different. While the 
mean vector remains the same, the variance-covariance structure of the parameter values is altered through the shifting of the location parameters.

The optimality criteria. As previously explained, the A- and D- optimality criteria have been selected for the purpose of test design optimisation on the premises of a group-based test setting.

Table 2 summarises the different conditions resulted from the cross-combinations of two factors, latent trait vector $\Theta$ and item pool ( 8 examinees groups, 4 item pools). They are supposed to mirror a broad spectrum of different situations from the practice of test construction. The total number of $\theta_{l}$ values in each specific examinees group $\Theta$ is given by $T$ in parenthesis. The bold numbers correspond to the respective graphics displayed in Figures 2 to 5. This means that Figure 2(a), for instance, displays the test information functions resulted from of an optimisation procedure based on the latent trait vector $\Theta_{N}$ with $T=300$ normally distributed $\theta_{t}$ values within the range $[-3,3]$, for which the test items have been selected from the item pool $I_{N}$.

Table 2. Overview of the combinations of the algorithm's conditions with respect to the vector of latent traits $\Theta$ and the item pools $I$.

\begin{tabular}{|c|c|c|c|c|c|}
\hline \multirow[b]{2}{*}{ Item pool } & \multicolumn{5}{|c|}{ Latent trait vector $\Theta$ and its range } \\
\hline & $\Theta$ & {$[-3,3]$} & {$[-3,-1]$} & {$[-1,1]$} & {$[1,3]$} \\
\hline \multirow[t]{2}{*}{$I_{N}$} & $\Theta_{\mathrm{N}}$ & $\begin{array}{l}\text { Fig. 2(a) } \\
(T=300)\end{array}$ & $\begin{array}{l}\text { Fig. 3(a) } \\
(T=44)\end{array}$ & $\begin{array}{l}\text { Fig. 4(a) } \\
(T=204)\end{array}$ & $\begin{array}{l}\text { Fig. 5(a) } \\
(T=52)\end{array}$ \\
\hline & $\boldsymbol{\Theta}_{\mathrm{U}}$ & $\begin{array}{l}\text { Fig. 2(b) } \\
(T=300)\end{array}$ & $\begin{array}{l}\text { Fig. 3(b) } \\
(T=94)\end{array}$ & $\begin{array}{l}\text { Fig. 4(b) } \\
(T=109)\end{array}$ & $\begin{array}{l}\text { Fig. 5(b) } \\
(T=97)\end{array}$ \\
\hline \multirow[t]{2}{*}{$J_{N}$} & $\Theta_{N}$ & $\begin{array}{l}\text { Fig. 2(c) } \\
(T=300)\end{array}$ & $\begin{array}{l}\text { Fig. 3(c) } \\
(T=44)\end{array}$ & $\begin{array}{l}\text { Fig. 4(c) } \\
(T=204)\end{array}$ & $\begin{array}{l}\text { Fig. 5(c) } \\
(T=52)\end{array}$ \\
\hline & $\Theta_{U}$ & $\begin{array}{l}\text { Fig. 2(d) } \\
(T=300)\end{array}$ & $\begin{array}{l}\text { Fig. 3(d) } \\
(T=94)\end{array}$ & $\begin{array}{l}\text { Fig. 4(d) } \\
(T=109)\end{array}$ & $\begin{array}{l}\text { Fig. 5(d) } \\
(T=97)\end{array}$ \\
\hline \multirow[t]{2}{*}{$I_{U}$} & $\Theta_{N}$ & $\begin{array}{l}\text { Fig. 2(e) } \\
(T=300)\end{array}$ & $\begin{array}{l}\text { Fig. 3(e) } \\
(T=44)\end{array}$ & $\begin{array}{l}\text { Fig. 4(e) } \\
(T=204)\end{array}$ & $\begin{array}{l}\text { Fig. 5(e) } \\
(T=52)\end{array}$ \\
\hline & $\Theta_{\mathrm{U}}$ & $\begin{array}{l}\text { Fig. 2(f) } \\
(T=300)\end{array}$ & $\begin{array}{l}\text { Fig. 3(f) } \\
(T=94)\end{array}$ & $\begin{array}{l}\text { Fig. 4(f) } \\
(T=109)\end{array}$ & $\begin{array}{l}\text { Fig. 5(f) } \\
(T=97)\end{array}$ \\
\hline \multirow[t]{2}{*}{$J_{U}$} & $\Theta_{\mathrm{N}}$ & $\begin{array}{l}\text { Fig. 2(g) } \\
(T=300)\end{array}$ & $\begin{array}{l}\text { Fig. 3(g) } \\
(T=44)\end{array}$ & $\begin{array}{l}\text { Fig. 4(g) } \\
(T=204)\end{array}$ & $\begin{array}{l}\text { Fig. 5(g) } \\
(T=52)\end{array}$ \\
\hline & $\Theta_{\mathrm{U}}$ & $\begin{array}{l}\text { Fig. 2(h) } \\
(T=300)\end{array}$ & $\begin{array}{l}\text { Fig. 3(h) } \\
(T=94)\end{array}$ & $\begin{array}{l}\text { Fig. 4(h) } \\
(T=109)\end{array}$ & $\begin{array}{l}\text { Fig. 5(h) } \\
(T=97)\end{array}$ \\
\hline
\end{tabular}




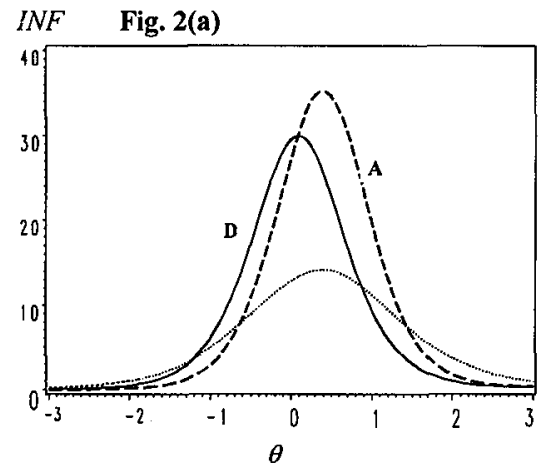

Fig. 2(c)

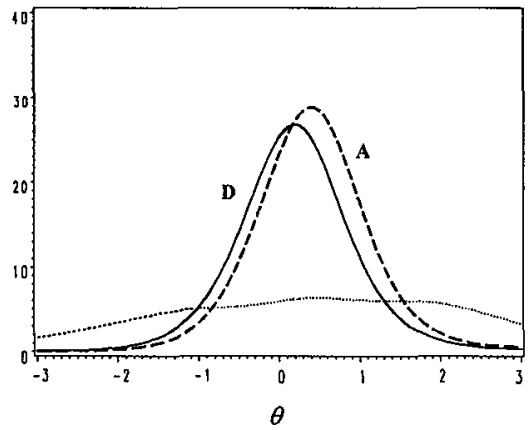

Fig. 2(e)

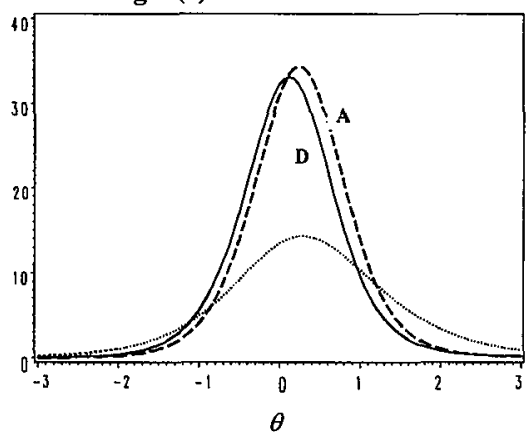

Fig. 2(g)

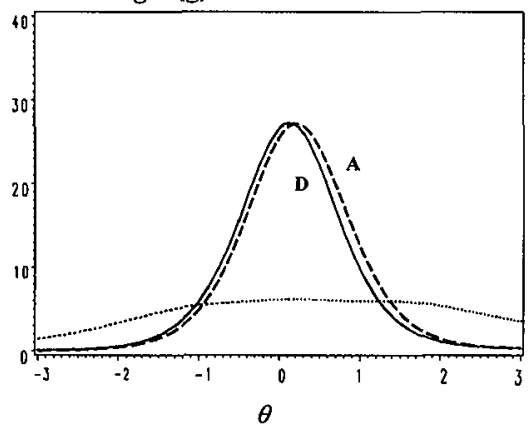

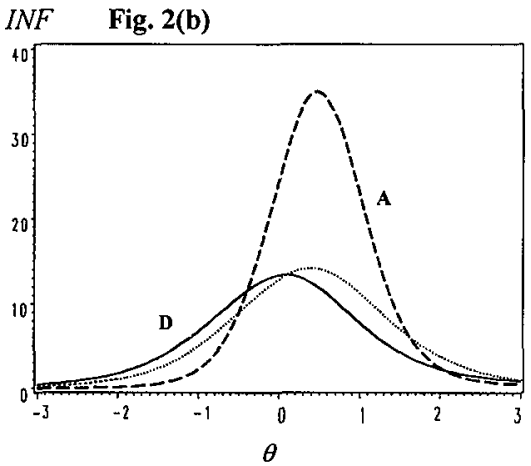

Fig. 2(d)

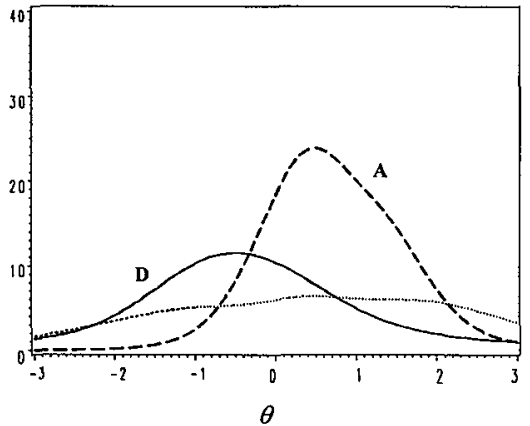

Fig. 2(f)

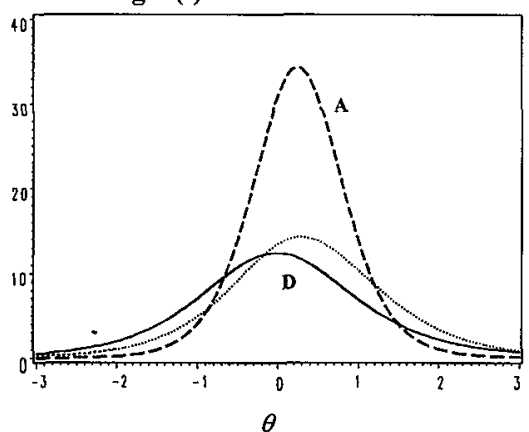

Fig. 2(h)

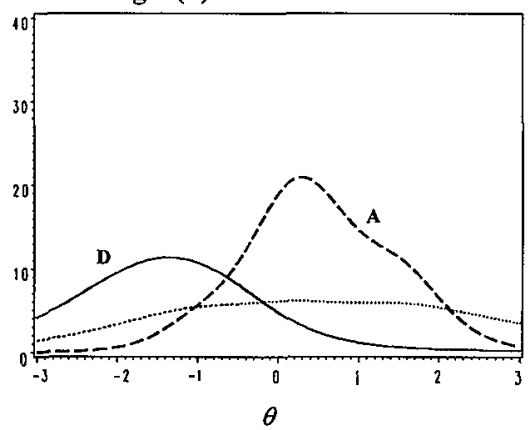

Figure 2. Test Information functions $(I N F)$ for the optimisation run on the $\theta$-range $=[-3,3]$ 


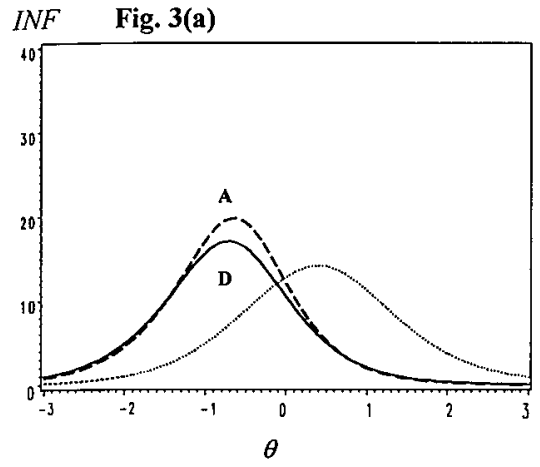

Fig. 3(c)

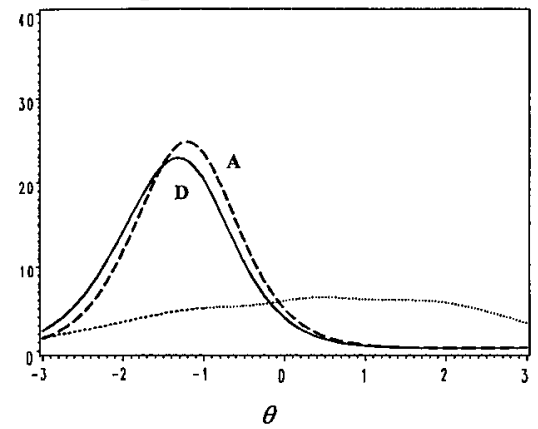

Fig. 3(e)

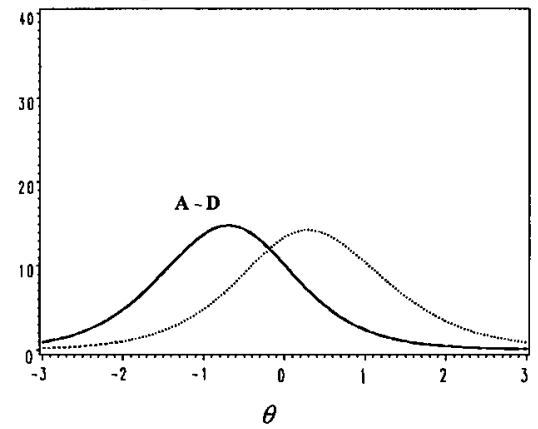

Fig. 3(g)

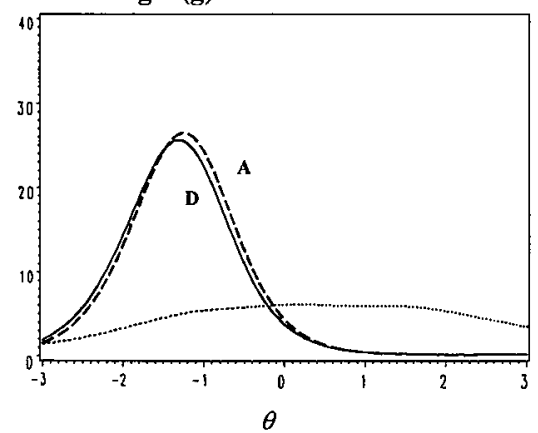

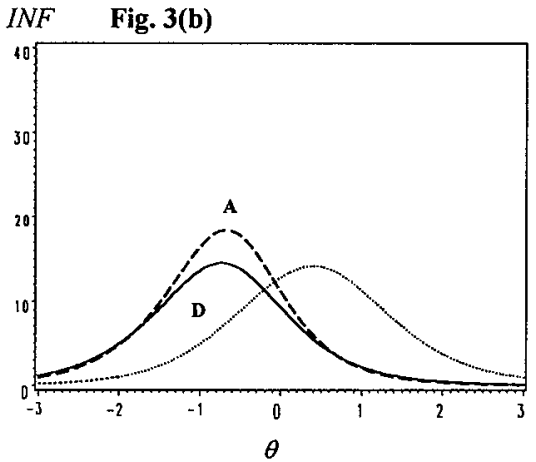

Fig. 3(d)

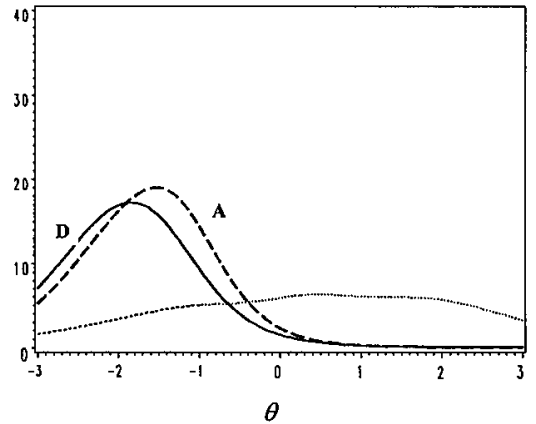

Fig. 3(f)

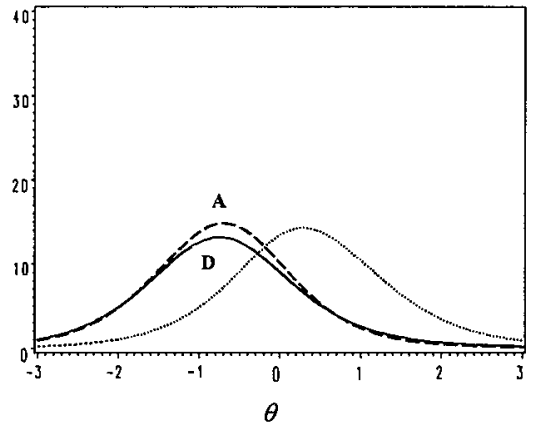

Fig. 3(h)

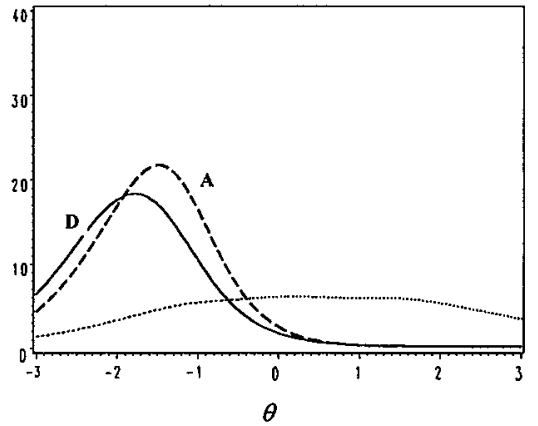

Figure 3. Test Information functions $(I N F)$ for the optimisation run on the $\theta$-range $=[-3,-1]$ 


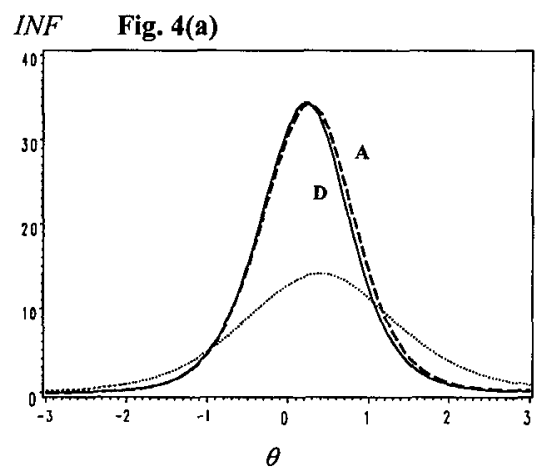

Fig. 4(c)

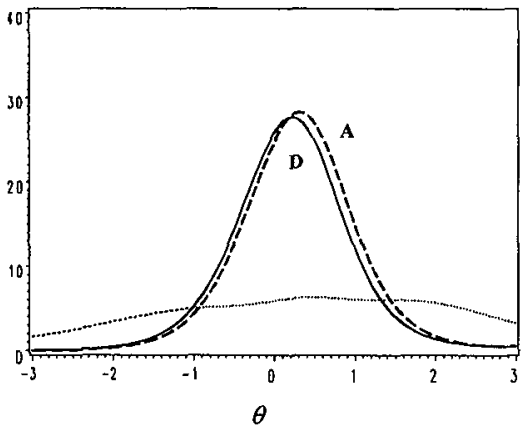

Fig. 4(e)

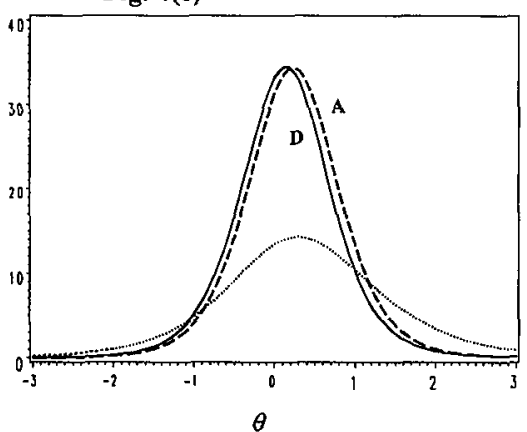

Fig. 4(g)

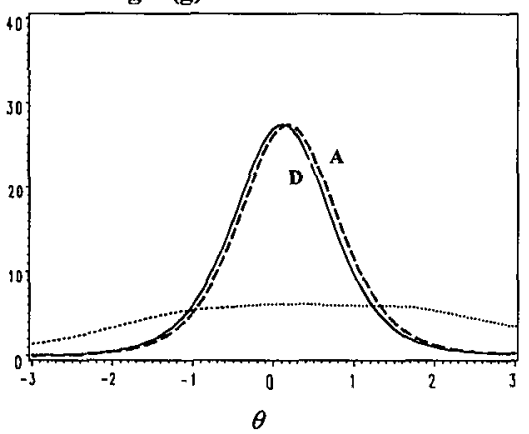

INF Fig. 4(b)

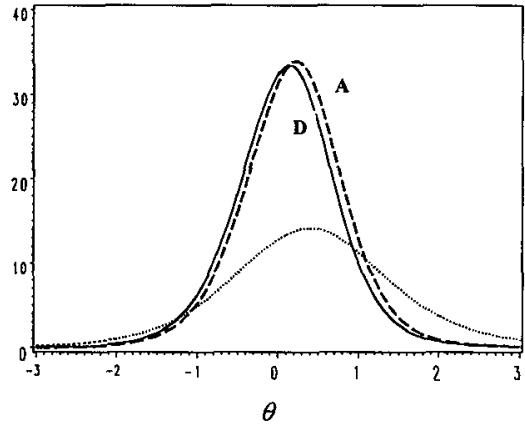

Fig. 4(d)

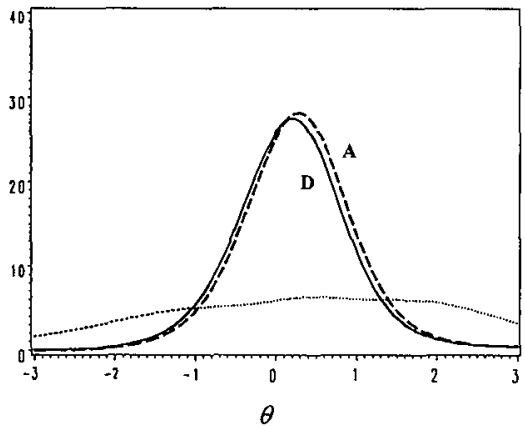

Fig. 4(f)

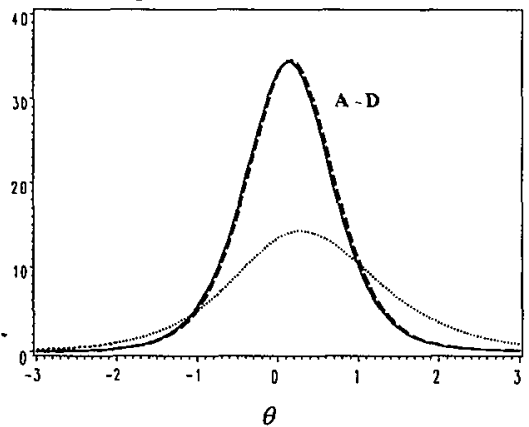

Fig. 4(h)

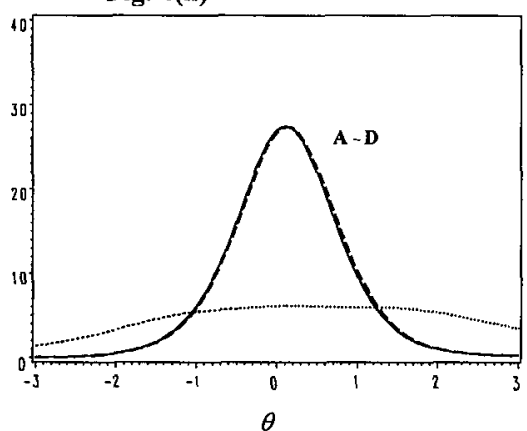

Figure 4. Test Information functions (INF) for the optimisation run on the $\theta$-range $=[-1,1]$ 


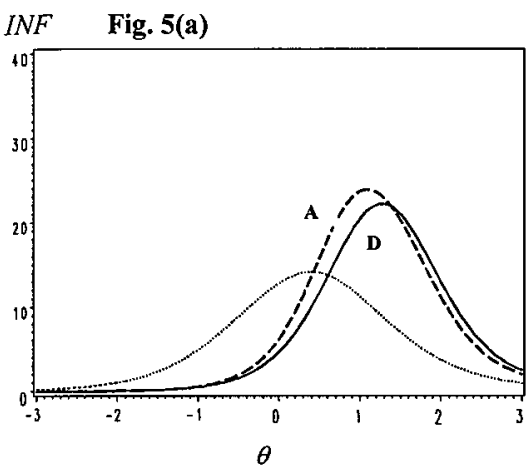

Fig. 5(c)

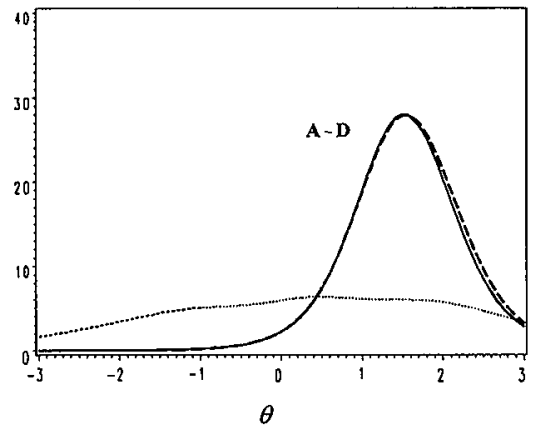

Fig. 5(e)

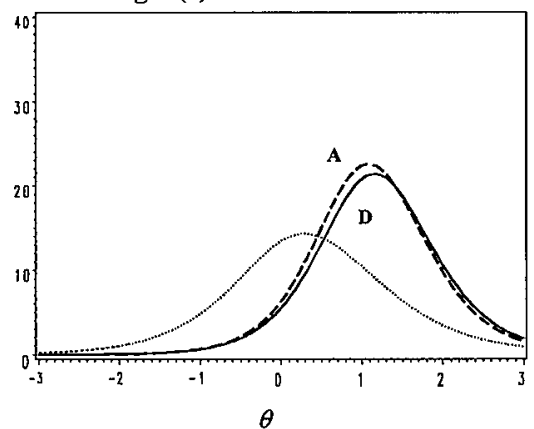

Fig. 5(g)

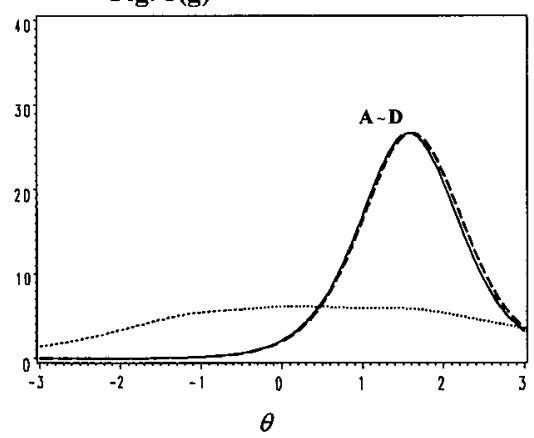

INF Fig. 5(b)

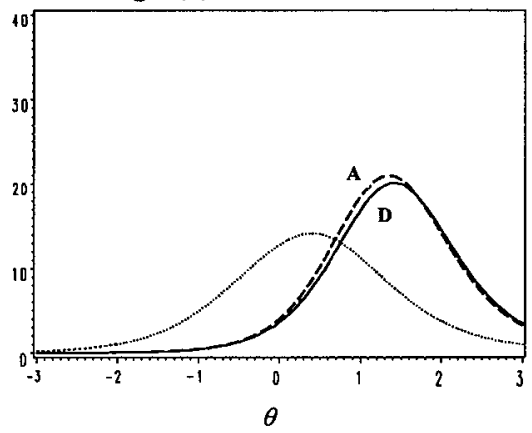

Fig. 5(d)

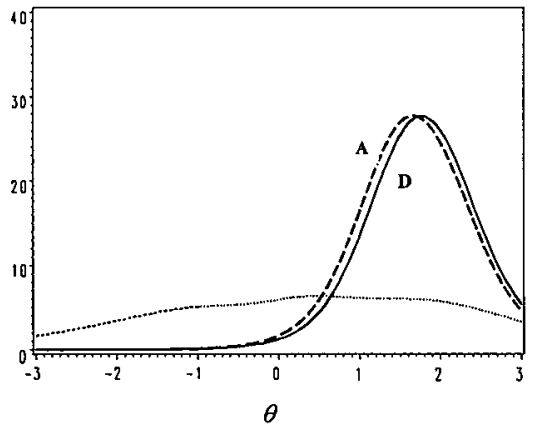

Fig. 5(f)

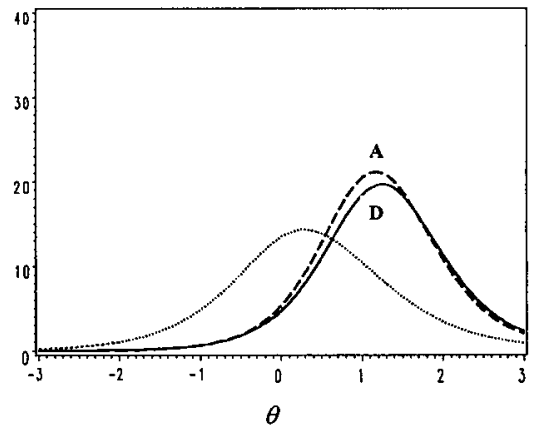

Fig. 5(h)

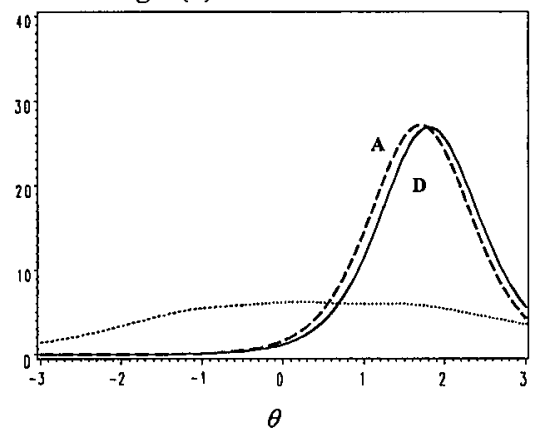

Figure 5. Test Information functions $(I N F)$ for the optimisation run on the $\theta$-range $=[1,3]$ 


\subsection{Results}

The results of in total 32 optimisation conditions are displayed graphically in Figures 2 to 5 in form of the final test information function $I N F$. Each graph contains 3 curves. Two of them, the interrupted and the full lines, represent the test information functions of the selected $n=17$ items based on the A- and D- optimality criterion, respectively. The fainted dotted line, on the other hand, has been added for comparative purposes. It can be thought as an example of a test function, whose 17 items have not undergone optimal selection. This reference curve is intended to convey a standard test representing an average of the pool's total information. For its representation, the item bank information function has been brought under the scale of the test information function given by 17 items. This curve has been calculated by summing all item information functions in the item pool, i.e. $I N F_{300}=\sum_{i=l}^{N} \operatorname{In} f_{i}$, where $N=300$, and eventually multiplying it by $17 / 300$, i.e. $I N F_{300} * 17 / 300$. The visual effect of this background curve is twofold: firstly, it enables a direct visualisation of how the test information function can be improved via optimisation. Moreover, it also reflects the specific items composition of the pool at stake. The reference line of the peaked pool resembles the test information function of the original 17 items, whereas for the flat pools, the curve is considerably flattened and broadened.

The differences between A-and D-optimality criteria emerge as especially conspicuous in Figure 2, where the latent trait vector has uniformly distributed values, $\Theta_{U}$ (Fig. 2(b), 2(d), 2(f) and 2(h)). These differences are much less evident for optimisation procedures run over the smaller ranges of the latent variables, i.e. the cases, in which the trait vector has a more homogeneous composition of trait levels (Figures 3 to 5). Here, the test functions produced by A- and D- criteria are more accordant, and in some cases even overlapping. Noticeable is also the shift of the curves as one goes from the lower (Figure 3) to the higher (Figure 5) subgroups of trait values, $\theta_{t} \in[-3,-1], \theta_{t} \in[-1,1]$ and $\theta_{t} \in[1,3]$, representing different ranges. This is a clear indication that the algorithm selects specifically those items to maximise the test information according to the underlying trait characteristics of the group of students. This optimisation on the narrower ranges shows furthermore how less informative the peaked item pools are for the extreme latent traits in comparison to the flat pools. By contrast, for the middle range (Figure 4) the peaked pools had more information. It seems that by generating items on shifted location parameters a trade-off took place: more informative items for extreme trait levels were obtained at the expense of less 
items for the middle $\theta$-values. This explains why the curves of the flat pools (Fig. 4(c), 4(d), 4(g) and 4(h)) are a little lower compared to the peaked (Fig. 4(a), 4(b), 4(e) and 4(f)). Still in association with the optimisations on the ranges $\theta_{t} \in[-3,-1], \theta_{t} \in[-1,1]$ and $\theta_{t} \in[1,3]$, it should be noted that the test information curves adjusted to the higher trait levels are comparatively higher (Figure 5) than for the low range (Figure 3). This is, once again, explained by the items' generation process that took the original 17 items as a blueprint. The majority of the original items have their information peaks around the middle range, favouring somewhat the higher trait levels (Figure 1). Consequently, more discriminative/informative items for this region have been generated.

Contrary to the results of Figures 3 to 5, the curves in Figure 2 display a greater discrepancy between the A- and D-optimality criteria for the latent trait vector $\Theta_{U}$ (Figure 2(b), 2(d), 2(f) and 2(h)). The A-criterion seems to favour items covering the middle trait range, regardless of the underlying distribution of trait values. If on the one hand, this result seems logical for the normal distribution of trait values, $\Theta_{N}$, where the vast majority of trait levels are concentrated in the middle, on the other, it is not that self-evident for $\Theta_{U}$. After all, in the latter case the traits localised on the tails of this distribution are strongly represented, and should, through their weight, exercise some influence on the items selection. This influence, however, is only visible for the D-optimality criterion, lending support to the idea that the latter is more sensitive to the frequency distribution of the trait values.

A similar phenomenon has been observed by Berger and van der Linden (1995) for dichotomous models. They described D- and A-optimality criteria in their weighting of trait distributions as conjunctive and disjunctive, respectively. The former seems to conjoin the weights of the trait values in the selection of items, while the latter does not.

On the grounds of these results it can be said that, for the given item pools, the Doptimality criterion tends to favour less discriminative items (low maximum information and wider tails), while the opposite is valid for the A-criterion (higher information peaks and lower tails). This claim is valid only under the precondition of a uniform distribution of trait levels in the heterogeneous group of students, i.e. a large underlying trait interval. This effect vanishes on the optimisations with more homogenous groups of trait values (Figures 3 to 5 ) and is much less evident for the optimisation based on $\Theta_{N}$ (Figures 2(a), 2(c), 2(e) and 2(g)). 


\subsection{The criteria selective mechanisms}

The best way of elucidating the differences between the A- and D-criteria is by means of a simple example, allowing for a more detailed scrutiny of the criteria different selective mechanisms.

Table 3 displays Fisher's Information values $\operatorname{In} f_{i, \theta_{i}}$ along the $\theta$-scale for three items selected out of the original 17 (Table 1) by the D-and A-optimality criteria. Two cases have been here differentiated. Firstly, the case in which the optimisation took place along a group of students, $T=7$, encompassing a greater range of trait values $\theta_{t} \in[-3,3]$ (Table 3-top). Alternatively, the optimisation has been run for a more homogeneous group, whose trait values $(T=7)$ are confined to a limited range $\theta_{l} \in[-1,1]$ (Table 3-bottom).

For both cases, item (9) is selected by the A-optimality criterion, whose information function is represented by the interrupted lines in Figure 6. For the D-criterion (full line), however, the two conditions resulted in different selected items, (7) and (10) for the wide and narrow trait range, respectively.

Making sense out of the differentiated selections by the D-optimality criterion can be achieved via an inspection of Table 3, where the steps involved in the calculation of the objective function $\Phi\left\{M\left(\Theta \mid a_{i}, c_{i}\right)\right\}$ are displayed for each selected item. The final row shows the resultant measurements of $\Phi\left\{\boldsymbol{M}\left(\Theta \mid a_{i}, c_{i}\right)\right\}$ that has been maximised (in bold). For the high discriminative item (9), the $\operatorname{Inf}_{i, \theta_{t}}$ values are substantially lower in the tails than for the low discriminative items (7). The $\log$ transformation of the low $\operatorname{Inf} f_{i, \theta_{i}}$ (item (9)) yields larger quantities in absolute values. These, in turn, considerably decrease the total sum, which defines the D-optimality criterion: $\Phi_{\mathrm{D}}\left\{\boldsymbol{M}\left(\Theta \mid a_{i}, \dot{c}_{i}\right)\right\}=\sum_{i=1}^{T} \log \operatorname{Inf} f_{i, \theta_{\mathrm{t}}}$, resulting in $\Phi_{\mathrm{D}}\{\boldsymbol{M}(\Theta \mid$ $\left.\left.a_{i}, c_{i}\right)\right\}=-24.011$, standing in contrast to the low discrimination item (7), for which $\Phi_{\mathrm{D}}\{M(\Theta \mid$ $\left.\left.a_{i}, c_{i}\right)\right\}=-13.845$. Since the objective of optimisation is to maximise $\Phi_{\mathrm{D}}\left\{M\left(\Theta \mid a_{i}, c_{i}\right)\right\}$ the item with lower discrimination (7), whose $\Phi_{\mathrm{D}}\left\{M\left(\Theta \mid a_{i}, c_{i}\right)\right\}=-13.845$, is obligatory selected. Once the whole trait range is considered, the D-optimality criterion tends to overvalue the low information of the tails for highly discriminative items, which eventually reduces the sum implicit in $\Phi_{\mathrm{D}}\left\{M\left(\Theta \mid a_{i}, c_{i}\right)\right\}$. The A-optimality criterion, by contrast, gives more emphasis to the maximum information value. Note, for instance, in Table 3 (top), that the information of item (9) on a single trait level $\theta_{l}=1\left(\operatorname{Inf} f_{i, \theta_{t}}=1.644\right)$ almost equalises the total information provided by the low discriminative item (7) for the whole $\theta_{\text {range, }} \Phi_{\mathrm{A}}\left\{\boldsymbol{M}\left(\Theta \mid a_{j}\right.\right.$, 
$\left.\left.c_{i}\right)\right\}=\sum_{i=1}^{T} \operatorname{In} f_{i, \theta_{i}}=1.696$. The A-criterion targets high peaks, which are usually associated with highly discriminative items. Yet they have the disadvantage of providing high information for a very small range of trait values.

Table 3. Items selected by the A- and D-optimality criteria with their respective Fisher's Information $\operatorname{Inf}$ i.

Fisher's Information and objective functions along the whole $\theta$-range $[-3,3]$ :

\begin{tabular}{|c|c|c|c|c|c|c|}
\hline & \multicolumn{2}{|c|}{ Item 9} & \multicolumn{2}{|c|}{ Item 7} & \multicolumn{2}{|c|}{ Item 10} \\
\hline$\theta_{l}$ & $\operatorname{Inf} f_{i, \theta_{1}}$ & $\log I n f_{i, O_{t}}$ & $\operatorname{Inf} f_{i, \theta_{t}}$ & $\log \operatorname{In} f_{i, 0_{1}}$ & $\operatorname{Inf} f_{i, \theta_{1}}$ & $\log I n f_{i . \theta_{t}}$ \\
\hline-3 & 0.000 & -9.080 & 0.020 & -3.902 & 0.002 & -6.214 \\
\hline-2 & 0.002 & -5.886 & 0.090 & -2.396 & 0.032 & -3.442 \\
\hline-1 & 0.067 & -2.702 & 0.343 & -1.068 & 0.331 & -1.105 \\
\hline 0 & 1.232 & 0.208 & 0.663 & -0.409 & 1.448 & 0.370 \\
\hline 1 & 1.644 & 0.497 & 0.408 & -0.896 & 0.584 & -0.601 \\
\hline 2 & 0.105 & -2.245 & 0.128 & -2.049 & 0.182 & -1.703 \\
\hline 3 & 0.008 & -4.801 & 0.044 & -3.123 & 0.037 & -3.296 \\
\hline$\Phi\left\{M\left(\Theta \mid a_{i}, c_{i}\right)\right\}$ & $\begin{array}{c}3.058 \\
(\mathrm{~A})\end{array}$ & $\begin{array}{c}-24.009 \\
\text { (D) }\end{array}$ & $\begin{array}{r}1.696 \\
(\mathrm{~A})\end{array}$ & $\begin{array}{c}-13.843 \\
\text { (D) }\end{array}$ & $\begin{array}{c}2.616 \\
(\mathrm{~A})\end{array}$ & $\begin{array}{c}-15.991 \\
\text { (D) }\end{array}$ \\
\hline
\end{tabular}

Fisher's Information and objective functions along a restricted $\theta$-range [-1,1]:

\begin{tabular}{|c|c|c|c|c|c|c|}
\hline & \multicolumn{2}{|c|}{ Item 9} & \multicolumn{2}{|c|}{ Item 7} & \multicolumn{2}{|c|}{ Item 10} \\
\hline$\theta_{l}$ & $\operatorname{In} f_{i, \theta_{t}}$ & $\log \operatorname{In} f_{i, \theta_{r}}$ & $\operatorname{Inf} f_{i, 0,}$ & $\log \operatorname{In} f_{i, \theta_{t}}$ & $\operatorname{Inf} f_{i, \theta_{l}}$ & $\log \operatorname{Inf} f_{i, \theta_{t}}$ \\
\hline-1 & 0.067 & -2.702 & 0.343 & -1.068 & 0.331 & -1.104 \\
\hline-0.75 & 0.146 & -1.919 & 0.443 & -0.814 & 0.552 & -0.592 \\
\hline-0.5 & 0.315 & -1.154 & 0.542 & -0.612 & 0.860 & -0.150 \\
\hline 0 & 1.232 & 0.208 & 0.663 & -0.409 & 1.448 & 0.209 \\
\hline 0.5 & 2.532 & 0.929 & 0.596 & -0.517 & 1.233 & -3.902 \\
\hline 0.75 & 2.357 & 0.857 & 0.507 & -0.679 & 0.895 & -0.110 \\
\hline 1 & 1.644 & 0.497 & 0.408 & -0.896 & 0.584 & -0.536 \\
\hline$\Phi\left\{M\left(\Theta \mid a_{i}, c_{i}\right)\right\}$ & $\begin{array}{c}8.293 \\
\text { (A) }\end{array}$ & $\begin{array}{r}-3.284 \\
\text { (D) }\end{array}$ & $\begin{array}{r}3.502 \\
(\mathrm{~A})\end{array}$ & $\begin{array}{r}-4.995 \\
\text { (D) }\end{array}$ & $\begin{array}{c}5.903 \\
(\mathrm{~A})\end{array}$ & $\begin{array}{c}-1.913 \\
\text { (D) }\end{array}$ \\
\hline
\end{tabular}


The contrast-aspect between very low and high informative parts of the information curve is no longer effective for the maximisation run on traits which are similar among themselves, as given by optimisations run on a smaller trait range (Table 3-bottom). This explains the selection of the slightly more discriminative item (10) by the D-criterion for the optimisation based on the $\theta$-range $[-1,1]$ (Figure 6(b)). Note, however, that the peak of the curve associated with the D-criterion (full) is still lower than that of the A-curve (interrupted).

Fig. 6(a) $\theta$-range $[-3,3]$ :

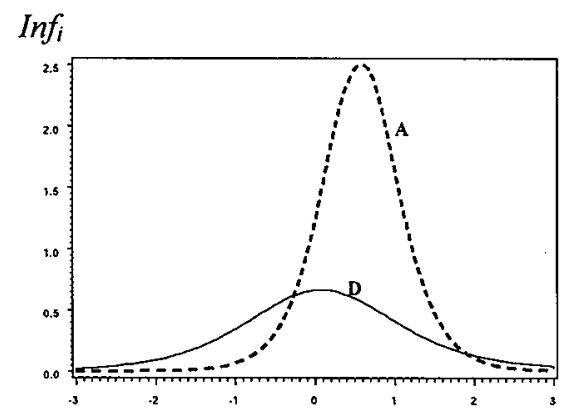

$\theta$
Fig. 6(b) $\theta$-range $[-1,1]$ :

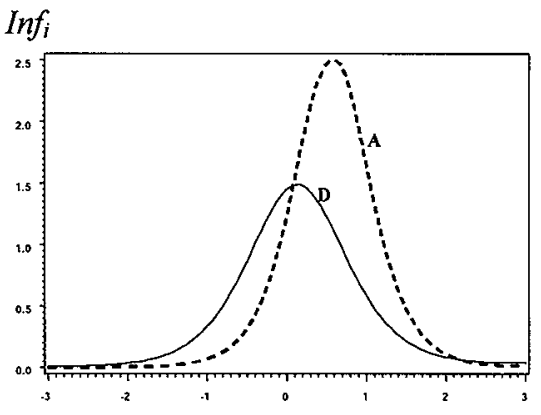

$\theta$

Figure 6. Item information functions

In the optimisation presented in Table 3 (top) all trait values $(T=7)$ are equally weighted. In this sense, it is comparable to the optimisations based on the uniform distribution of trait values, $\boldsymbol{\Theta}_{U}$ (Figures $2(\mathrm{~b}), 2(\mathrm{~d}), 2(\mathrm{f})$ and $2(\mathrm{~h})$ ), explaining by analogy the flattening of the information curve of the D-optimal test. However, for the cases with $\Theta_{N}$, the D-optimality criterion clearly selected items with higher information peaks, in spite of the wide range of trait values [-3,3] (see Figures 2(a), 2(c), 2(e) and 2(g)). This outcome can be traced back to the weighting effect. The overwhelming majority of $\theta_{t} \in \Theta_{N}$ is concentrated around the middle range, for which, moreover, the item pools, and especially the peaked pools $(I)$ have the most informative items. The more weights a group of trait levels has, the more information on this level is included in the criterion value (Berger and Mathijssen, 1997). This seems to be especially the case for the D-optimality criterion. As a result, its tails-driven inclination was compensated and surpassed by the weighting around the central traits. 


\subsection{Concluding remarks}

For a group-based test, this study has demonstrated the ways of how the selective mechanism of an optimality criterion interacts with the frequency distribution of the latent traits in the choice of optimal items, conditional on the quality of the item pool composition. We have seen that for a fixed-form, group-based test, the A-and D-optimality criteria have, in principle, different selective mechanisms. However, the degree to which they converge or diverge in their final items selection is influenced by the sample of test-takers. Questions related to how homogeneous the group is in terms of the latent trait levels as well as their frequency distribution are of major importance. As a whole, the more homogeneous the groups, the more overlapping the tests assembled by the A- and D-optimality criteria. Their differences may, but does not necessarily come to the fore, once the group of students is diversified. Here the traits frequency distribution can counteract or alternatively buttress the fundamental differences between the two criteria.

Van der Rijn et al. (2002) have shown for the graded response model that an item selection is independent of the underlying traits, if it is based on the sum of Fisher's information measures along a wide trait range (i.e. the A-criterion). A criterion based on the rule 'maximise the sum of equally weighted information measures along a large trait interval', becomes only dependent on the items discrimination parameters, picking the item with the highest $a_{i}$ value. The results presented for the NRM point to a similar explanation behind the selection by the A-criterion. A reversed situation seems to apply to the Doptimality criterion, which manifests a greater sensitivity to the latent trait distribution.

These findings substantiate the line of argument presented in the introduction, namely that an a priori knowledge of a criterion's modus operandi can be rather helpful in making the decision about which criterion to apply for the test assembly. For this purpose, the criteria preferences need to be pondered with the specific goal of the test, which can be translated into a target for the test information function (van der Linden, 1998). In a pass-fail decision test, for which high information on certain trait level is required (selection decision with cut score), the A-optimality criterion is appropriate. This criterion safeguards maximum information for the trait threshold of interest. The draw back, however, is that the estimation of trait levels, which are not in the vicinity of the threshold, can be rather unreliable. For grading or the progress tests, by contrast, the selected items are expected to provide sufficient information for a wide spectrum of latent trait values. For such tests, test-makers can always recourse to the classical solution for linear tests, which maintain high values of 
discrimination parameters, but space the value of location parameters (van der Linden and Pashley, 2000). Alternatively, the D-optimality criterion may be a suitable option.

It has been seen that, even after optimisation, a common shortcoming of fixed-form, groups based tests remains: they cannot provide high and at the same time broad amount of information for all trait levels. An alternative to this impasse is individually, tailored tests, as in computerised adaptive testing (CAT). Generally speaking, the issue related to the choice of an item selection criterion earns greater prominence in CAT literature. Yet, the Doptimality criterion has not been so far considered. Due to its accentuated dependence on the underlying trait distribution, its applicability in a CAT setting can be anticipated. This, however, is subject of further investigation. 


\section{Appendix: Optimal test design algorithm for NRM items}

\section{Starting premises:}

An item bank $I$ containing $N$ items with known parameters, with $a_{i}=\left[a_{i 1}, \ldots, a_{i g}, \ldots, a_{i k_{i}}\right], c_{i}=\left[c_{i 1}, \ldots, c_{i g}, \ldots, c_{i k_{i}}\right]$, and $i=1, \ldots, N$, A pre-determined test length $n$, A pre-specified number of $T \theta_{t}$-values, represented by the vector $\Theta$, whose elements $\theta_{t}$ follow a probability distribution, e.g. $\theta_{l} \sim N(0,1)$ or $\theta_{l} \sim U n(-3,3)$, with $\Theta=\left[\theta_{1}, \theta_{2}, \ldots, \theta_{\Gamma}\right]$ and $t=1, \ldots, T$.

\section{Notation:}

$\operatorname{Inf} f_{i, \theta_{t}}$ is Fisher's information of item $i \in I$ on a single $\theta_{l}$.

$\log I n f_{i, 0_{t}}$ is the natural logarithm of Fisher Information of item $i \in I$ on a single $\theta_{l}$. $M\left(\Theta \mid a_{i}, c_{i}\right)=\left[\operatorname{Inf} f_{i, \theta_{1}}, \operatorname{Inf} f_{i, \theta_{2}}, \operatorname{Inf} f_{i, \theta_{3}}, \ldots, \operatorname{Inf} f_{i, \theta_{T}}\right]$ is a vector of Fisher's information of an item $i$ on all traits $\theta_{i} \in \Theta$.

$\Phi\left\{M\left(\Theta \mid a_{i}, c_{i}\right)\right\}$ is the objective function of Fisher's Information:

D-optimality criterion: $\Phi_{\mathrm{D}}\left\{\boldsymbol{M}\left(\Theta \mid a_{i}, c_{i}\right)\right\}=\prod_{t=1}^{T} \operatorname{Inf}_{i, \theta_{t}} \Leftrightarrow \sum_{t=1}^{T} \log \operatorname{Inf}_{i, \theta_{t}} \quad$ (i.e. the sum of the $\log$ of Fisher information values of an item $i$ over $\Theta$ ).

A-optimality criterion: $\Phi_{\mathrm{A}}\left\{\boldsymbol{M}\left(\Theta \mid a_{i}, c_{i}\right)\right\}=\sum_{i=l}^{T} \operatorname{In} f_{i, \theta_{t}}$ (i.e. the sum of Fisher information values of an item $i$ over $\Theta$ ).

$\sum_{i=1}^{n} \Phi\left\{M\left(\Theta \mid a_{i}, c_{i}\right)\right\}$ is the objective function of Fisher information of an item $i$ summed over the $n$ constituent items of the test.

\section{Sequential Algorithm:}

Steps:

1. For each $i \in I$, calculate its Fisher information $\operatorname{Inf}_{i, \theta_{t}}$ on all $\theta_{t} \in \Theta$, 
2. Compute $\Phi\left\{M\left(\Theta \mid a_{i}, c_{i}\right)\right\}$, i.e. the sum, or alternatively the sum of the $\log$ of Fisher information values of all $N$ items $i \in I$, over $\Theta$, according to the selected optimality criterion,

3. Select the $n$ largest out of $N$ values of $\Phi\left\{M\left(\Theta \mid a_{i}, c_{i}\right)\right\}$ after completion of step 2.

The sum of Fisher information values $\sum_{i=1}^{n} \Phi\left\{M\left(\Theta \mid a_{i}, c_{i}\right)\right\}$ is maximised.

\section{Possible test constraints}

The above algorithm can be changed to allow for the inclusion of test constraints. The optimisation target becomes, thus:

Maximise $\sum_{i=1}^{n} \Phi\left\{M\left(\Theta \mid a_{i}, c_{i}\right)\right\} x_{i}$, with $x_{i}=1$ in case the item is selected and $x_{i}=0$, if left out. This expression can be subject to specifications like $\sum_{i=1}^{n} w_{i} x_{i} \leq W$, with $W$ representing the maximum number of words allowed in the test, or, for instance, $\sum_{i=1}^{n} x_{i} \leq n_{c}$ for $c=1, \ldots, \mathrm{C}$, with $c$ giving a content classification. 


\section{Test design optimisation in CAT early stage with the Nominal Response Model}

This chapter has been reviewed by Applied Psychological Measurement, and is undergoing revision for publication (co-authors M.P.F. Berger and F.E.S. Tan). 


\title{
Test Design optimisation in CAT early stage with the Nominal Response Model
}

\begin{abstract}
The early stage of CAT is a term used to denote the instable phase of trait estimation after the administration of the first few items. In this study, two procedures are presented in an attempt to lessen this instability. A first one involves a change in the test format. A small-sized fixed-test is introduced as a starting premise for the subsequent sequential items administration, a test-form referred to as semi-adaptive. A second procedure relates to the application of an item selection criterion hitherto not used in connection with CAT: the D-optimality criterion. A polytomous CAT simulation is carried out to evaluate this criterion's performance under different test premises. Other established criteria based on Fisher's and Kullback-Leibler information are considered for comparative purposes. The simulation shows that the extent of early stage instability depends primarily on the quality of the item pool information and its size, and secondarily on the item selection criteria. A comparison between a fully and semiadaptive tests reveals that the latter can considerably lessen the instability. The efficiency of D-optimality criterion is similar to the efficiency of other known item selection criteria, often yielding more stable estimates along the course of items administration.
\end{abstract}

Keywords: CAT early stage, polytomous IRT model, Test Design optimisation, Item selection criteria 


\section{Test Design Optimisation in CAT early stage with the Nominal Response Model}

\subsection{Introduction}

The amount of information an item provides along the latent trait range is a major quantification tool in computerised adaptive testing (CAT). It enables a selection of items to best meet the demands of a test's objective, efficient and accurate estimation of an individual trait, $\theta$. For this purpose, items are sequentially selected to match the updated estimator, $\hat{\theta}$. More recently, CAT algorithms have been increasingly applied in decision-making situations, in which an accurate/efficient classification of test-takers into proficiency categories is envisaged (Eggen, 1999, Weissman, 2003). In either case, the fine-tuning between trait level and item takes place through an item selection criterion (ISC).

Enhancing $\hat{\theta}$ 's efficiency is often achieved by picking an item whose specific value of Fisher's Information (FI) on the provisional point estimator $\hat{\theta}$ is maximised. Though widely applied, this FI or maximum information criterion has a few shortcomings. A most discussed one, known as the attenuation paradox (Lord and Novick, 1968, sect.16.5, van der Linden and Pashley, 2000), represents a serious hindrance in the early stages of CAT, where the estimators' variance is still relatively high and the estimator $\hat{\theta}$ might strongly deviate from the true trait value $\theta_{0}$. Consequently, a mismatch between the items, selected to fit the newly updated estimator, and true value can arise, leading to further inefficiency of the succeeding $\hat{\theta}$.

To circumvent this problem, alternative item selection criteria (ISC) have been introduced. The basic idea behind these ISCs is to allow for the information to be measured not only with respect to a single point $\hat{\theta}$, but rather to an interval built around the latter. The proposed alternative criteria can be seen as extended variants of either FI, as a measurement of local information (Veerkamp and Berger, 1997), or of the Kulback-Leibler (KL), a global information criterion (Chang and Ying, 1996). Bayesian ISCs, on the other hand, require an informative prior distribution of the true trait value and are based on the posterior variance of the estimator. For further details in this topic see Owen (1975), Berger and Veerkamp (1996), van der Linden (1998) and van der Linden and Pashley (2000).

Several studies (Chen et al., 2000, Cheng and Liou, 2000, van der Linden and Pashley, 2000, van Rijn et al. 2002, Veldkamp, 2003) have shown that, as the number of administered 
items increase, both alternative as well as traditional FI criteria yield accurate and precise $\theta$ estimation. This means that, for a large number of items, the differences among these criteria performances are negligible. For a small numbers of items, however, it has been argued that the global information seems more appropriate to counteract the attenuation paradox. A settled consensus on this subject does not seem to have been established yet. One study supports this conjecture (Chang and Ying, 1996), while another seems to have found no empirical evidence to back it up (Fan and Hsu, 1996).

In the current work, the question of criteria's efficiency at the early stage of CAT will be investigated for items described by the Nominal Response Model (NRM). The objective is to identify suitable 'starting conditions' for a CAT under different test specifications. For this purpose, the following approach will be introduced. Contrary to usual CAT implementations, which commonly start with one item tailored to a central $\theta$-value, i.e. $\theta=0$, and moves on stepwise from that, a fixed number of five initial items will be simultaneously selected by different criteria. The strictly adaptive part of the test starts after these initial items have been answered and a provisional $\hat{\theta}$ obtained. This early stage represented by a fixed number of items is hereafter referred to as semi-adaptive, standing in contrast to the fully adaptive CAT.

As regards the item selection, emphasis is to be laid on three ISCs: The D- and Aoptimality criteria and the $\mathrm{KL}$ information criterion. D-optimality criterion has been previously applied in CAT in the context of constrained multidimensional test assembly with positive results (Veldkamp, 2001). However, a detailed evaluation of its selective preferences and performance in unidimensional CAT has not been yet undertaken.

The paper is divided in two parts. The first one entails a comparative evaluation of the criteria performances within the semi-adaptive approach solely. The second part places the preceding investigation into an adaptive setting, in which items are sequentially selected. The simulation study is carried out for both the fully and the semi-adaptive approaches. The criteria's performances will be eventually measured via the accuracy (bias) and efficiency (MSE). Firstly, however, a short description of the NRM and the Information measures.

\subsection{The Nominal Response Model and Information Measures}

\subsubsection{The Nominal Response Model}

The Nominal Response Model (NRM) was firstly introduced by Bock (1972). The NRM is considered the most general model in the divide-by-total model classification 
category (Thissen and Steinberg, 1986). It does not require the ordering of categories to reflect different degrees of the trait level to be measured. It is, therefore, suitable for nominal data (Heinen, 1996, p.103). Furthermore, the NRM can model not only polytomous, but also dichotomous data, being equivalent to the 2PL model, when responses are treated in two categories (De Ayala, 1993). Additionally, as Thissen and Steinberg (1986) have shown, the NRM and Partial Credit Model (PCM) are also equivalent when proper constraints are imposed on the NRM. The equation that describes the NRM is:

$$
P_{i g \mid \theta_{t}}=\frac{\exp \left(c_{i g}+a_{i g} \theta_{t}\right)}{\sum_{h=1}^{k_{i}} \exp \left(c_{i h}+a_{i h} \theta_{l}\right)}
$$

where $a_{i g}$ is the discrimination parameter of category $g$ of item $i, i=1,2, \ldots, n, c_{i g}$ is the location parameter associated with category $g$ of item $i, k_{i}$ is the number of categories of item $i$ with $g=1,2, \ldots, k_{i}, \theta_{t}$ is the latent trait variable of examinee $t$, with $t=1,2, \ldots, T$, and $P_{i g \mid \theta_{i}}$ is the probability of examinee with trait $\theta_{t}$ of choosing category $g$ of item $i$.

\subsubsection{Fisher's Information}

Fisher's information is the amount of information supplied by the data on an unknown parameter $\theta$. It is defined as:

$$
\operatorname{Inf}=-E\left(\frac{\partial^{2} \log L}{\partial \theta^{2}}\right)
$$

where $L$ is the likelihood of a sample of $n$ independent item response observations, with respect to which the expectation $E$ is found. The part of the $\log$ likelihood function, $\log L$ that is usually maximised in order to obtain the maximum likelihood (ML) estimates is given by:

$$
\sum_{t} \sum_{i} \sum_{g} f_{i t g} \log P_{i g \mid \theta_{t}}
$$


where $f_{i t g}$ is the number of examinees with ability $\theta_{t}$ that respond to each of the $k_{i}$ categories of item $i$. The probability $P_{i g \mid \theta_{1}}$ is defined in equation (1). For the NRM, Fisher's Information of an item $i$ on a single $\theta_{l}$ (Heinen, 1996) is given by:

$$
\operatorname{Inf}_{i}=\left\{\sum_{g} a_{i g}^{2} P_{i g \mid \theta_{t}}-\left[\sum_{g} a_{i g} P_{i g \mid \theta_{i}}\right]^{2}\right\} .
$$

The index $g$ runs through the number of categories $k_{i}$ of item $i$, i.e. $g=1,2, \ldots, k_{i}$ with $a_{i g}$ standing for their corresponding discrimination parameters.

Fisher's information expresses the discrimination power between two trait levels that are close together. In this context, for any given trait value $\theta_{0}$, Fisher's information conveys the local information that an item contains around a single $\theta_{0}$ (Chang and Ying, 1996; Chen et al, 2000).

\subsubsection{Kullback-Leibler Information}

The use of Kullback-Leibler (KL) information in CAT has been suggested by Chang and Ying (1996). KL information function of an item $i$ is based on a ratio between two likelihoods and measures the distance between them. The larger this value, the easier it is to discriminate between the likelihoods, which is the same as to say between the parameters that characterise them:

$$
K_{i}\left(\theta, \theta_{0}\right)=E\left(\log \frac{L\left(\theta_{0}\right)}{L(\theta)}\right)
$$

As in the case of Fisher's Information, $E$ denotes the expectation over the observed item responses. For a polytomous IRT model the KL Information for a single item $i$ can be expressed as:

$$
K_{i}\left(\theta, \theta_{0}\right)=\sum_{g} P_{i g \mid \theta_{0}} \log \left(\frac{P_{i g \mid \theta_{\theta}}}{P_{i g \mid \theta}}\right) .
$$


Contrary to FI, KL information is a function not of one, but of two trait levels. It should be noted that, by having the true trait value $\theta_{0}$ fixed, the other one, $\theta$, can be made to vary along the whole $\theta$ range. In this case the KL information becomes a function. To obtain a KL information criterion that, similarly to the $\mathrm{FI}$ is given by a scalar-index, Chang and Ying (1996) proposed integrating $\mathrm{KL}$ function, over a given interval $\left[\theta_{\mathrm{L}}, \theta_{\mathrm{U}}\right]$. The $\mathrm{KL}$ information criterion is described at greater length in the following section.

\subsection{Item selection criteria (ISC)}

Van den Linden and Pashley (2000) provide a detailed overview of CAT item selection criteria with their merits and limitations. Generally speaking, there seems to be so far no established consensus about which criterion is the most adequate. Recent techniques developed for the CAT environment indicate that the issue of optimal item selection is more suitably approached in a practice-specific, instead of global optimal context.

In the present paper, three ISCs stand in the spotlight, the D-and A-optimality criteria and the KL information criterion. The first two criteria are well known in the area of optimal experimental design (Aktinson and Donev, 1992). Berger and Veerkamp (1996) and Berger and Mathijssen (1997) apply them in the specific context of design optimisation for fixedform, group-based tests. So far, the D-optimality criterion has not been explicitly used in connection with CAT. Its viability in an adaptive setting will be investigated by means of a comparison with the A-optimality and the $\mathrm{KL}$ information criterion, which has become a standard alternative to criteria based on Fisher's Information.

Both A- and D-optimality criteria are objective functions $\Phi($.$) of an information$ measure. In test design optimisation this function $\Phi$ needs to be maximised along a latent trait interval $\Theta=\left[\theta_{\mathrm{L}}, \theta_{\mathrm{U}}\right]$, with $\theta_{t} \in \Theta$,

$t=1,2, \ldots t, \ldots, T$. Thus $t$ is the index that runs through the elements $\theta_{l} \in \Theta$. $\theta_{L}$ and $\theta_{U}$ represent the interval's lower and upper boundaries, with $t=1$ and $t=T$, respectively. The Aoptimality, trace or sum criterion, is given by:

$$
\Phi_{\mathrm{A}}\left(\ln f_{i}\right)=\sum_{i=1}^{T} \ln f_{i}
$$

where Inf $f_{i}$ is Fisher's information of item $i$ on a single $\theta_{l}$, as defined in (2), The A-optimality criterion is a weighted sum of information measures, where the weights are given by the 
frequency distribution of $\theta_{t} \in \Theta$. For the present study, all $\theta_{l} \in \Theta$ are equally weighted within the interval. It should be noted that $\Theta$ 's lower and upper boundaries, $\theta_{L}$ and $\theta_{U}$, will be systematically changed as to provide different starting premises for the semi-adaptive CAT implementation.

The product or determinant or D-optimality criterion is defined as:

$$
\Phi_{\mathrm{D}}\left(\operatorname{Inf} f_{i}\right)=\prod_{i=1}^{T} \ln f_{i} .
$$

Berger and Veerkamp (1996) conceptually describe the A-criterion as correspondent to the arithmetic mean, and the $\mathrm{D}$-criterion to the geometric mean. In a more general setting of optimal experimental designs, the D-optimality criterion has been shown to have rather advantageous properties (see Aktinson and Donev, 1992, and Berger and Veerkamp, 1996). It is inversely proportional to the volume of $\hat{\theta}$ 's confidence ellipsoid as well as invariant under any linear transformation of the independent variable scale. For test design optimisation, this is to say the D-optimal design remains the same irrespective of the scale used to measure the latent trait variable, $\theta$. Berger and van der Linden (1995) have found that for fixed test forms with dichotomous items, the item selection by the $\mathrm{A}$ and D-optimality criteria can be quite different. Similar results have been found by Lima Passos et. al. (2003) for the NRM. Distinct selective mechanisms of the two criteria have been reported, indicating a more accentuated dependence of the D-optimality criterion on the distribution of the underlying trait values. Contrary to the A-, the D-optimality criterion seems to target items with relatively low discrimination parameters, mainly when the trait interval, upon which the optimisation is run, is large.

Finally, the global information criterion (KL) is also considered. Chang and Ying (1996) proposed an ISC that integrates $\mathrm{KL}$ function, $K_{i}\left(\theta, \theta_{0}\right)$, over a given interval $\left[\theta_{\mathrm{L}}, \theta_{\mathrm{U}}\right]$, where $\theta_{\mathrm{L}}$ and $\theta_{\mathrm{U}}$ are the lower and upper interval boundaries, respectively, and the true trait value $\theta_{0}$ is fixed and known with $\theta_{0} \in \Theta=\left[\theta_{\mathrm{L}}, \theta_{\mathrm{U}}\right]$ :

$$
K_{i}\left(\theta_{0}\right)=\int_{\theta_{L}}^{\theta_{U}} K_{i}\left(\theta, \theta_{0}\right) d \theta
$$


The area under the curve of the KL function associated with the trait interval $\Theta$ represents the average information index to be maximised. The measure $K_{i}\left(\theta_{0}\right)$ indicates how powerful a specific item is in holding other trait values from the true one apart. It summarises information content of an item with respect to a broad spectrum of $\theta$-levels (Chang and Ying, 1996).

Part 1 of this paper concerns only the early stage within the semi-adaptive premise, without involving any trait estimation. Thus, for the sake of consistency, the interval applied for the A- and D-optimality criteria, i.e. the trait interval $\Theta$ will be also used to calculate $K_{i}$. In this case, $\theta_{0}$ needs to be specified in advance and will be represented by the central value in $\Theta$.

\subsection{Part 1 - An early stage investigation}

The aim of administrating a few numbers of items at once in the semi-adaptive test is twofold. Firstly, starting premises are sought, which offer similar, and, if possible, a sufficient amount of information to fairly cover all trait values within the range of interest. Secondly, a more stable early stage is anticipated once the estimation is based on the response pattern of a few items instead of only one.

\subsubsection{Number of items in the early stage}

The early stage of CAT is a term associated with the relative instable phase of $\theta$ estimation after the administration of only a few items. This denomination begs the question of how many items it should entail. The relevant asymptotic properties of estimators, like normal distribution and variance (given by the inverse of the test information function), are not expected to hold in this phase. Samejima (1977), however, suggested that the normality is satisfactory for a test of 10 to 20 items (Samejima, 1977, Chen et al., 2000). The asymptotic variance, on the other hand, is not as easily met. The estimator's standard error after 10, 20 items is larger than the ideal lower bound (Chen et al. 2000).

A common result from previous works on ISCs (see for instance, Chen at al, 2000, Cheng and Liou, 2000 and van der Linden and Pashley, 2000), is that bias and MSE are, regardless of the ISC, still high for the first 10 items. Based on the existent empirical data, $n=10$ could be regarded as a rough upper boundary delimiting the early stage of an adaptive test. For the present study, however, the fixed number of items to be simultaneously selected 
is $n=5$. In this phase, the estimation instability is more accentuated. Results with the simultaneous selection of $n=10$ have been also obtained. They strongly resemble the ones with $n=5$. To avoid redundancy, they are omitted.

\subsubsection{Item selection criteria}

The simultaneous selection of $n=5$ items for a CAT early stage is carried out by the Aand D-optimality criteria and the $\mathrm{KL}$ information criterion. The resulting test information functions are denoted $I N F_{\mathrm{A}}, I N F_{\mathrm{D}}$ and $I N F_{\mathrm{KL}}$, respectively. These information functions are computed as the sum of the individual item information functions, i.e. $I N F=\sum_{i=1}^{n} \operatorname{Inf} f_{i}$, where $n=5$ is the number of selected items. For comparative purposes, two additional criteria are also applied. A benchmark is given by the random selection of $n=5$ of items, yielding $I N F_{\mathrm{r}}$. A 'systematic' selection of items is also considered by picking $n=5$ equidistant $\theta$ points along the pre-defined trait interval $\Theta=\left[\theta_{\mathrm{L}}, \theta_{U}\right]$, and by selecting for each point the corresponding optimal item, i.e. the one that punctually yields the maximum information. The resulting test information function is denoted $I N F_{\mathrm{s}}$. In total, 5 criteria are used for the concurrent selection of the initial 5 items, yielding the test functions: $I N F_{\mathrm{A}}, I N F_{\mathrm{D}}, I N F_{\mathrm{KL}} I N F_{\mathrm{r}}$ and $I N F_{\mathrm{s}}$.

\subsubsection{Widths of the trait interval $\Theta$}

In the present paper the following widths of the latent trait interval $\Theta$ will be considered: $[-3,3],[-1,1],[-3,0],[0,3]$. These are intervals of discrete equally weighted $\theta$ values. The $\Theta$ widths, upon which the item selection is based, can be conceptually linked to both tests' premises and objectives. The test premises, for instance, could be the presence of any prior knowledge about the examinee's trait level. This prior knowledge (or lack thereof) is expressed in terms of the trait range covered by the interval. As for the test objectives, the trait range can also convey the group of traits targeted by the test, i.e. a narrow, pass-fail decision test, or a test, for which the whole spectrum of trait levels, such as $[-3,3]$, is of relevance. 


\subsubsection{Item pools}

Four basic item pools are used. Two of them, denoted $I_{300}$ and $J_{300}$, contained 300 items $(N=300)$. These two pools differ basically in their total information dispersion and coverage along the trait range. Additionally two larger pools, $I_{600}$ and $J_{600}(N=600)$, yet analogous to the previous ones in terms of trait coverage, have been taken into account.

For item pools $I_{300}$ and $I_{600}$, the parameters values of the $300 / 600$ items $i \in I$, with $N=300(600)$, have been generated by multivariate normal distribution, using 17 calibrated items as a blue print. These items stem from the discipline of internal medicine of the socalled Progress tests, annually administered at the University of Maastricht. They are considered to be representative of items found in practice.

The final item bank information functions, computed as a sum of the 300/600 item information functions is represented in Figure 1. Also displayed is the item bank information function for the item pools $J_{300}$ and $J_{600}$. The generation of the parameters values of $J$ succeeded similarly to $I$. The difference lies on the location parameter values $c_{i g}$, which have been intentionally shifted to the left and right along the $\theta$-scale. As a result, a broader coverage of the $\theta$-range in terms of information has been achieved, yet with a lower information peak.

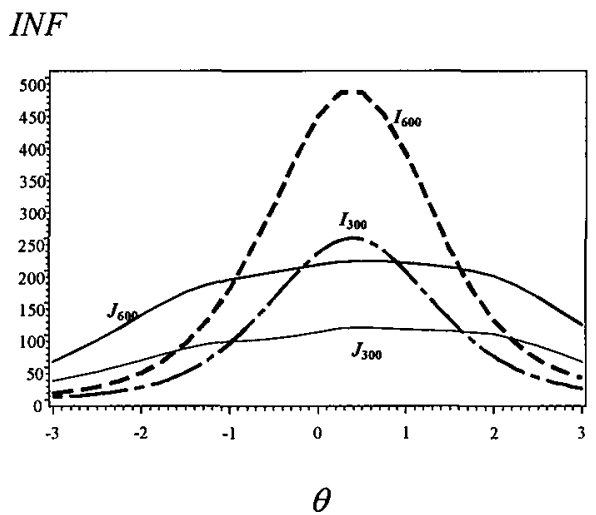

Figure 1. Item bank information for four item pools $I_{300}, J_{300}, I_{600}, J_{600}$

\subsubsection{Measuring the criteria performances}

Let it be reminded that so far the trait values are known. This means that for each $\theta_{l} \in \Theta$, with $\boldsymbol{\Theta}=[-3,3]$ an optimal, fully adaptive set of 5 items can be found, based on the maximum information (point) criterion (FI), yielding $I N F_{\max }$. The maximum information, 
which is dependent on the quality of the item pool, is the highest that can be possibly achieved by the full-adaptive selection of $n$ items, i.e. $I N F_{\text {ISC }} \leq I N F_{\max }$.

Knowledge of both $I N F_{\max }$ and $I N F_{\text {ISC }}$ allows for a quantitative evaluation of the criteria's performance in terms of information provided by the early stage fixed test. This will be based on two indexes expressing either the amount of absolute or relative information.

Absolute Information. The area under the curve of the test information function of an ISC, $\mathrm{AUC}_{I N F_{\mathrm{ISC}}}$, conveys the expected amount of absolute information on the trait value given by the selected items, corrected for the $\Theta$ width and is defined as:

$$
\mathrm{AUC}_{I N F_{\mathrm{ISC}}}=\int_{\theta=O_{L}}^{\theta_{U}} I N F_{\mathrm{ISC}} d \theta
$$

Relative Information (relative efficiency). The expected absolute information provides the grounds, upon which the choice of the most suitable criterion can be based. Alternatively, this choice can be equally done with a relative measure of information, hereafter referred to as relative efficiency.

The relative efficiency is defined as a ratio between $I N F_{\text {ISC }}$ and $I N F_{\max }$ at one specific trait level $\theta_{l} \in \Theta$ :

$$
e f f_{\theta_{t}}(\mathrm{ISC})=\frac{I N F_{\mathrm{ISC}}\left(\theta_{t}\right)}{I N F_{\max }\left(\theta_{t}\right)}
$$

Analogously to (11) the area under the relative efficiency curve $\left(\mathrm{AUC}_{e f f_{\mathrm{sc}}}\right)$ with

$$
\mathrm{AUC}_{e f f_{\mathrm{ISC}}}=\int_{\theta=\theta_{L}}^{\theta_{U}} e f f_{\mathrm{ISC}} d \theta
$$

can be taken as an index measure of the criterion's expected efficiency over the $\theta$ range. The larger this $\mathrm{AUC}_{\text {effisc }}$, the closer the selected items are to the optimal situation. As in the case of absolute information, the objective of optimisation is to maximise this $\mathrm{AUC}_{\text {eff } \mathrm{ISC}}$. 


\subsubsection{Results}

For illustrative purposes, a few test information functions of the selected initial $n=5$ items are shown in Figures 2(a) to 7(a) together with the full-adaptive curve (the upper curve). An interpolation of the information-maxima $\left(I N F_{\max }\right)$ obtained for each $\theta_{l} \in \Theta$ along the $\theta$ scale yields the full-adaptive curve. The other curves in Figures 2(a) to 7(a) represent the preliminary test information functions $I N F_{\text {ISC }}$ based on the initial items as selected by the ISCs. Attention needs to be drawn to the fact that for large trait intervals (Figures 2(a) and 3(a)) the differences between the produced test information functions by the A-, D- and KL criteria are more evident than for the smaller intervals (Figures 4(a) to 7(a)). Note above all the flat curve of the D-criterion information function for the large trait intervals, compared to the A- and KL-curves (Figures 2(a) and 3(a)). This mirrors the selection of relatively low discriminative items by the $\mathrm{D}$-optimality criterion, as opposed to the more discriminative items by the $\mathrm{A}$ and $\mathrm{KL}$ criteria.

A graphical representation of the ISCs relative efficiency curves along the $\theta$-scale can be seen in Figures 2(b) to 7(b). The plane eff $\mathrm{x} \theta_{l}$, corresponds to $100 \%$ efficiency along the $\theta$ interval of interest.

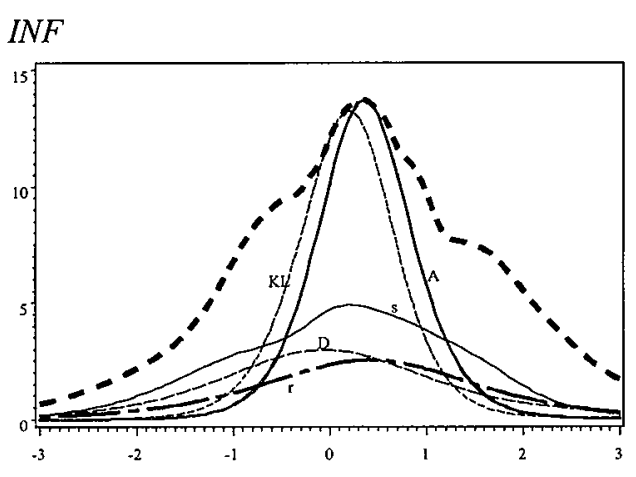

$\theta$

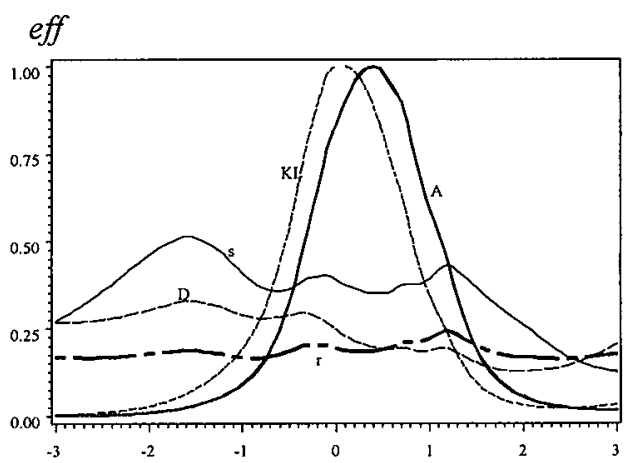

$\theta$

2(a). $I N F_{\text {ISC }}$ curves

2(b). Relative efficiency curves

Figure 2. Item pool $I_{300}$, trait interval $\Theta=[-3,3]$. 


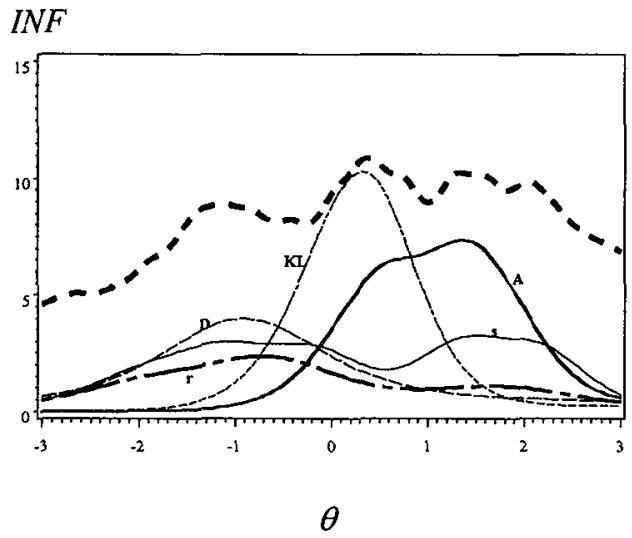

3(a). $I N F_{\text {ISC }}$ curves

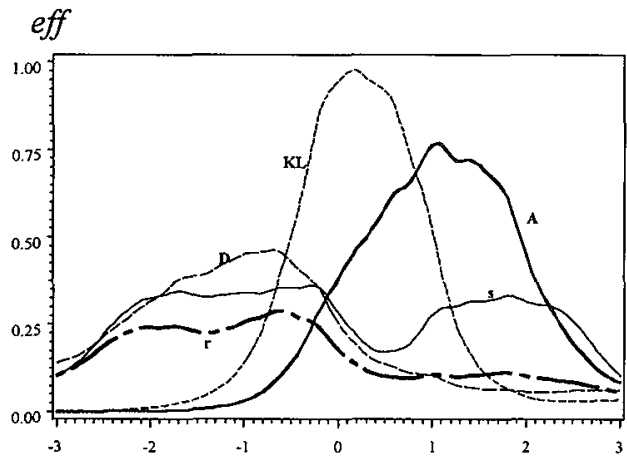

$\theta$

Figure 3. Item pool $J_{300}$, trait interval $\Theta=[-3,3]$.

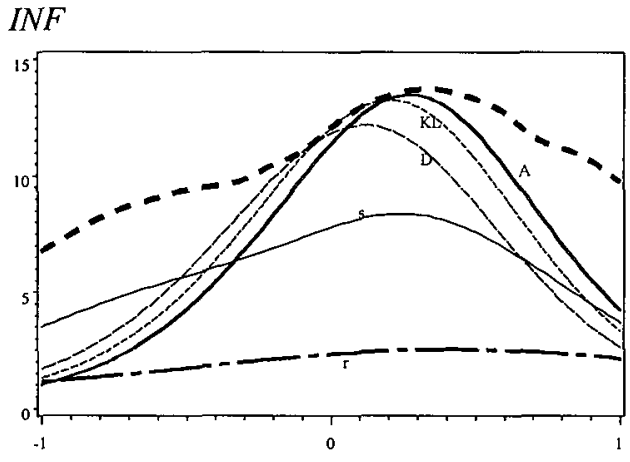

$\theta$

4(a). $I N F_{\text {ISC }}$ curves

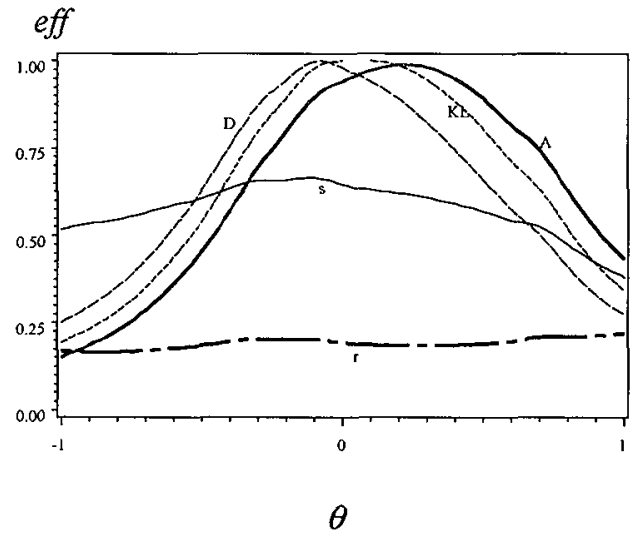

4(b). Relative efficiency curves

Figure 4. Item pool $I_{300}$, trait interval $\Theta=[-1,1]$.

In Tables 1 to 2, the absolute information of the five different ISC are displayed in brackets, and the largest values highlighted in bold. Provided is also a reference in terms of maximum absolute information adjusted for the interval width (last column in Tables 1 to 2), given by the area under the full-adaptive curve, $\mathrm{AUC}_{I N F_{\max }}$.

The relative efficiency eff is defined with respect to the maximum amount of information that can be possibly obtained from the available item bank, whereas the absolute 


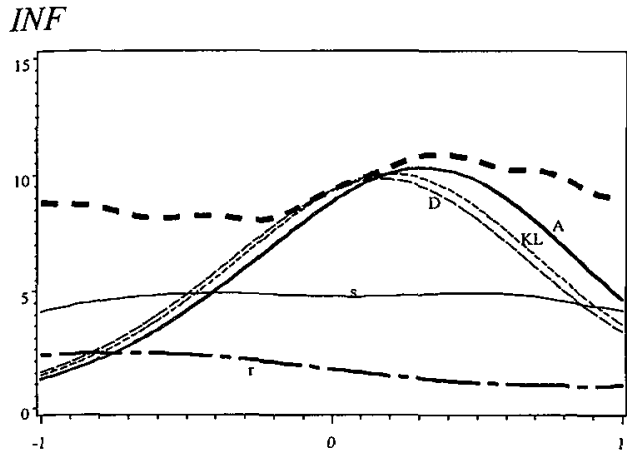

$\theta$

5(a). $I N F_{\text {ISC }}$ curves

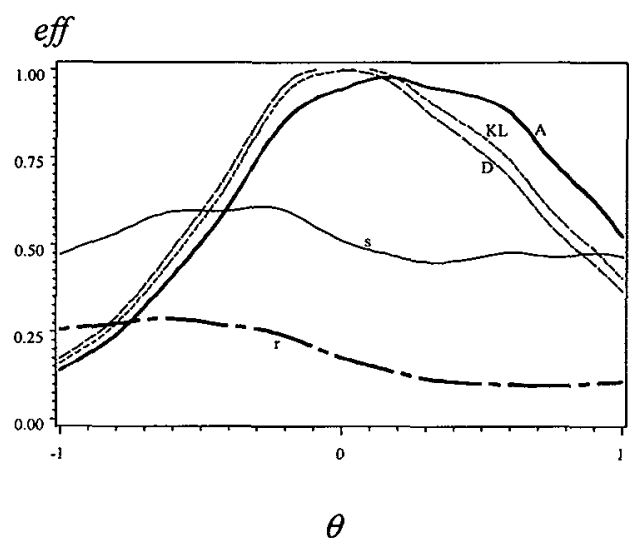

5(b). Relative efficiency curves

Figure 5. Item pool $J_{300}$, trait interval $\Theta=[-1,1]$.

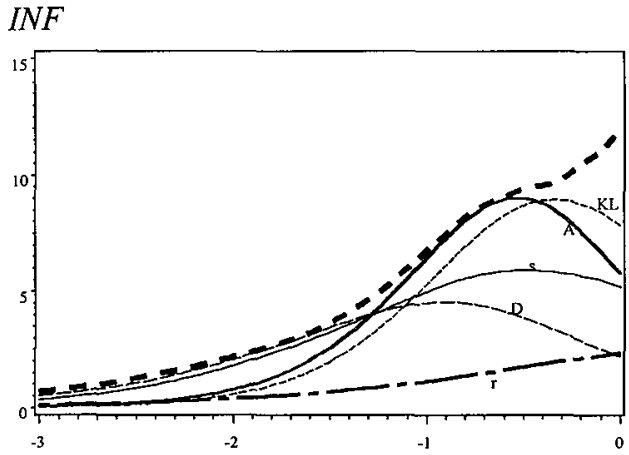

$\theta$

6(a). $I N F_{\text {ISC }}$ curves

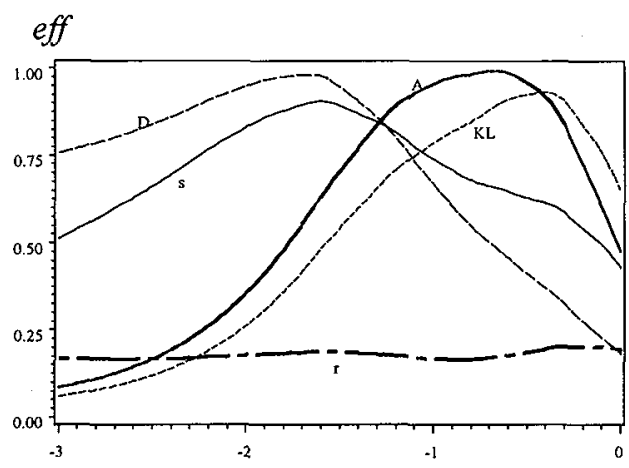

$\theta$

Figure 6. Item pool $I_{300}$, trait interval $\Theta=[-3,0]$.

6(b). Relative efficiency curves

INF makes no reference to it. This referential basis makes eff a standardised performance measurement, since it eliminates the discrepancies of how the information is spread over the trait range. This effect is visible, for instance, in the contraposition of Figures 6(a) and 6(b). In Figure 6(a), the $I N F_{\mathrm{D}}$, has a much lower peak than $I N F_{\mathrm{A}}$ and $I N F_{\mathrm{KL}}$ and smaller $A U C_{I N F_{\mathrm{D}}}$ than $A U C_{I N F_{\mathrm{A}}}$ and $\mathrm{AUC}_{I N F_{\mathrm{KL}}}$, i.e. it is less informative in absolute terms for a trait region [-3,0] (Table 1: $\mathrm{AUC}_{I N F_{\mathrm{D}}}=2.74, \mathrm{AUC}_{I N F_{\mathrm{A}}}=3.72$ and $\mathrm{AUC}_{I N F_{\mathrm{KL}}}=3.48$ ). And yet, in terms of $\mathrm{AUC}_{I N F_{\mathrm{D}}}$, the D-criterion manages to cover almost $72 \%$ of the $100 \%$ area of optimal 


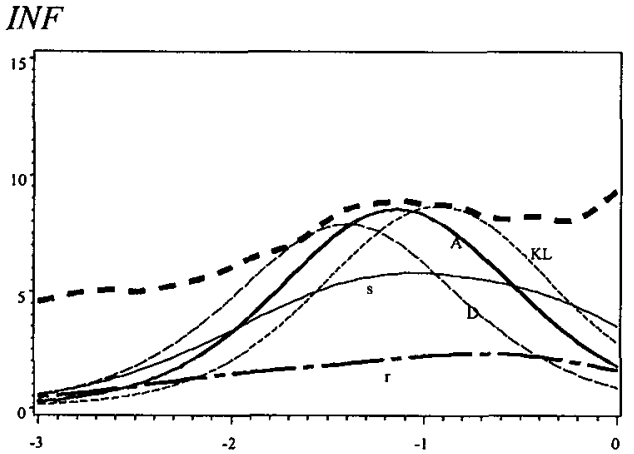

7(a). $I N F_{\text {ISC }}$ curves

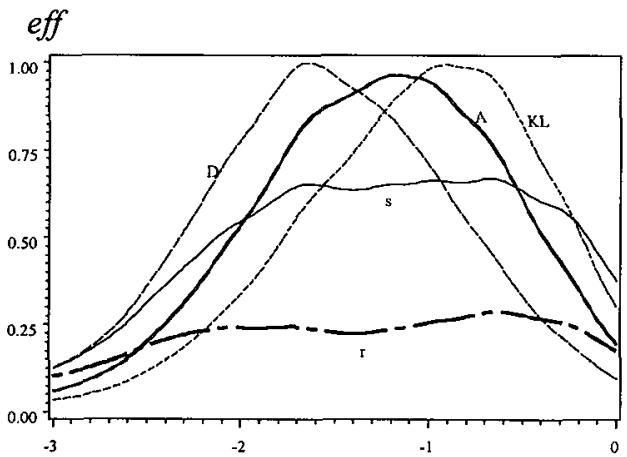

7(b). Relative efficiency curves

Figure 7. Item pool $J_{300}$, trait interval $\Theta=[-3,0]$.

Table 1. Relative and absolute information (in brackets) - Item pools with 300 items

\begin{tabular}{|c|c|c|c|c|c|c|c|}
\hline \multirow[b]{2}{*}{$\Theta$} & \multirow[b]{2}{*}{ pool } & \multicolumn{5}{|c|}{ ISC } & \multirow[b]{2}{*}{$\mathrm{AUC}_{I N F_{\max }}$} \\
\hline & & $r$ & $\mathbf{s}$ & A & $\mathrm{D}$ & $\mathrm{KL}$ & \\
\hline$[-3,3]$ & $\begin{array}{l}I_{300} \\
J_{300}\end{array}$ & $\begin{array}{c}0.183 \\
(1.19) \\
0.163 \\
(1.31)\end{array}$ & $\begin{array}{l}\mathbf{0 . 3 5 4} \\
(2.30) \\
\mathbf{0 . 2 7 6} \\
(2.31)\end{array}$ & $\begin{array}{l}0.264 \\
(\mathbf{2 . 8 8}) \\
0.274 \\
(\mathbf{2 . 6 3 )}\end{array}$ & $\begin{array}{l}0.231 \\
(2.13) \\
0.215 \\
(1.72)\end{array}$ & $\begin{array}{l}0.265 \\
(2.82) \\
0.274 \\
(2.59)\end{array}$ & $\begin{array}{l}6.289 \\
8.264\end{array}$ \\
\hline$[-1,1]$ & $\begin{array}{l}I_{300} \\
J_{300}\end{array}$ & $\begin{array}{l}0.191 \\
(2.08) \\
0.186 \\
(1.66)\end{array}$ & $\begin{array}{l}0.577 \\
(6.29) \\
0.517 \\
(4.74)\end{array}$ & $\begin{array}{l}0.660 \\
(7.68) \\
0.690 \\
(6.54)\end{array}$ & $\begin{array}{c}0.646 \\
(7.29) \\
0.670 \\
(6.28)\end{array}$ & $\begin{array}{l}\mathbf{0 . 6 6 8} \\
(7.68) \\
0.676 \\
(6.36)\end{array}$ & $\begin{array}{l}10.86 \\
9.260\end{array}$ \\
\hline$[-3,0]$ & $\begin{array}{l}I_{300} \\
J_{300}\end{array}$ & $\begin{array}{l}0.178 \\
(0.89) \\
0.225 \\
(1.65)\end{array}$ & $\begin{array}{l}0.658 \\
(3.11) \\
0.516 \\
(3.89)\end{array}$ & $\begin{array}{l}0.581 \\
(3.72) \\
0.557 \\
(4.32)\end{array}$ & $\begin{array}{l}\mathbf{0 . 7 1 8} \\
(2.74) \\
0.547 \\
(4.02)\end{array}$ & $\begin{array}{l}0.507 \\
(3.48) \\
0.525 \\
(4.20)\end{array}$ & $\begin{array}{l}4.856 \\
7.136\end{array}$ \\
\hline$[0,3]$ & $\begin{array}{l}I_{300} \\
J_{300}\end{array}$ & $\begin{array}{l}0.189 \\
(1.50) \\
0.101 \\
(0.96)\end{array}$ & $\begin{array}{l}0.506 \\
(4.01) \\
0.462 \\
(4.42)\end{array}$ & $\begin{array}{l}0.453 \\
(\mathbf{4 . 7 1}) \\
\mathbf{0 . 5 2 5} \\
\mathbf{( 5 . 0 1 )}\end{array}$ & $\begin{array}{l}\mathbf{0 . 5 8 9} \\
(4.10) \\
0.491 \\
(4.75)\end{array}$ & $\begin{array}{l}0.431 \\
(4.69) \\
0.460 \\
(4.54)\end{array}$ & 7.722 \\
\hline
\end{tabular}


Table 2. Relative and absolute information (in brackets) - Item pools with 600 items

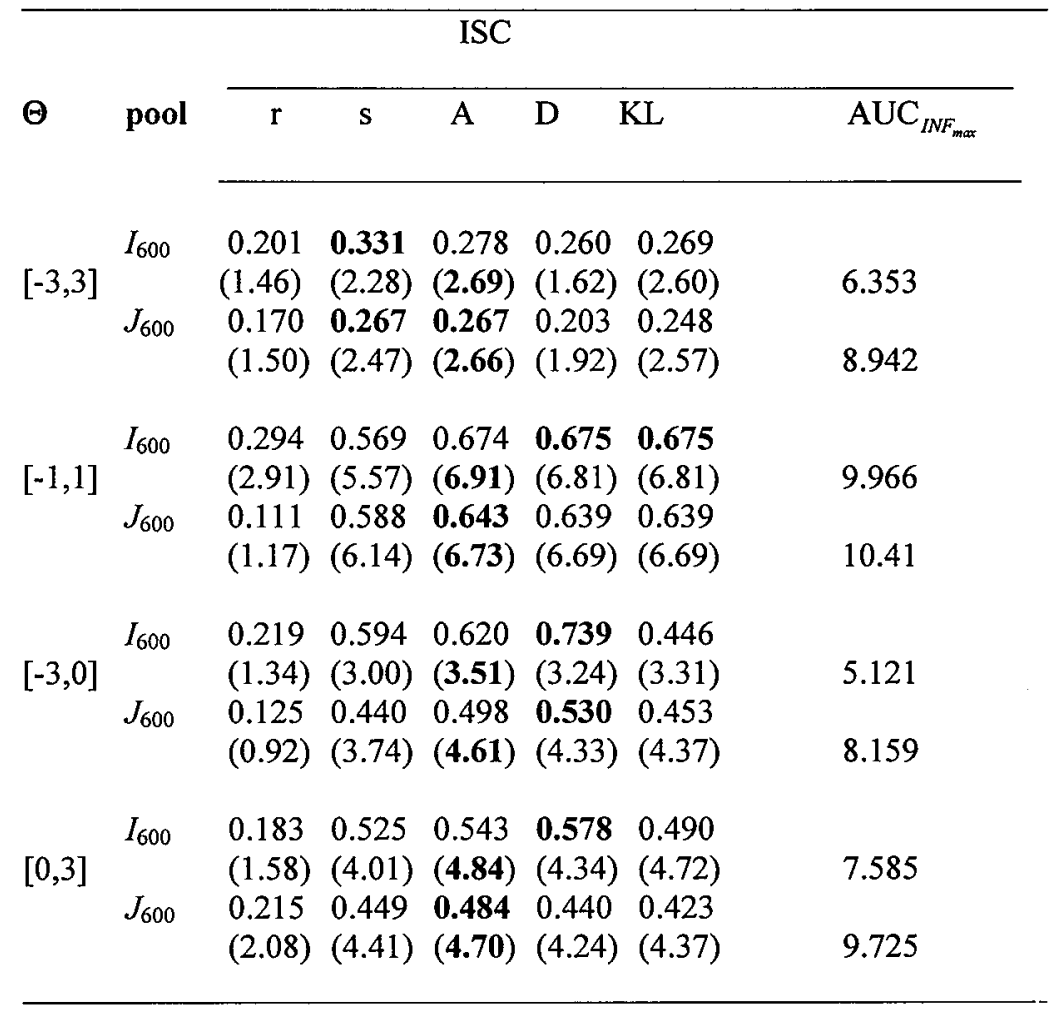

efficiency (Figure 6(b)), while these values are $58 \%$ and $50 \%$ for the A- and KL criteria, respectively. Note, however that, contrary to the absolute information, from the $\mathrm{AUC}_{\text {eff }}$ isc values alone, little can be gathered about how much information the items are actually delivering.

These results suggest an upper-hand of the A-optimality criterion in terms of absolute information, irrespective of the item pool size/quality and targeted trait range. The picture changes slightly for relative efficiency. Here, the systematic criterion, for instance, seems to make the most efficient use of the available information for the large trait interval. The final decision on which of the two information measures is the most adequate for picking the best criterion is not simple and needs to be above all supported by empirical evidence. This is the objective of the following simulation study. For the CAT simulation, only the interval $\Theta=[-$ $3,3]$ is considered for the CAT initialisation. 


\subsection{Part 2: The simulation study}

The CAT simulation is carried out to investigate the ISCs performances at the early CAT stage in terms of efficiency and precision. At the same time, these performances are evaluated against a background of the fully versus the semi-adaptive approach.

\subsubsection{Item selection criteria and the trait interval $\Theta$}

The ISCs are the same as described in section 4, i.e. the A- and D- optimality criteria and the $\mathrm{KL}$ global information criterion. These three criteria are defined as a function of a trait interval $\Theta=\left[\theta_{\mathrm{L}}, \theta_{\mathrm{U}}\right]$, upon which the maximisation of an item information measure takes place. In a CAT setting this interval is updated at each step of the CAT procedure.

Veerkamp and Berger (1997) proposed assimilating the uncertainty of the estimator $\hat{\theta}$ in each step of the CAT by using its confidence interval. The criterion can be formulated as the area under the curve of the information function of an item $i$ on all trait values from the lower and upper boundaries of the interval $\theta_{\mathrm{L}}$ to $\theta_{\mathrm{U}}$ (Veerkamp and Berger, 1997). It should be emphasised that the maximum interval information (MII) criterion is a discrete version of the A-optimality criterion defined in (7).

The KL information criterion (6) is per definition associated with an interval around the known true value $\theta_{0}$. Because $\theta_{0}$ is unknown, Chang and Ying (1996) proposed replacing $\theta_{0}$ by its provisional trait estimator $\hat{\theta}$ and calculating the integral over an interval on the neighbourhood of $\hat{\theta},\left[\hat{\theta}-\delta_{k}, \hat{\theta}+\delta_{k}\right]$, where $\delta_{k}$, the confidence limit, is inversely proportional to the rank number of the item in CAT. This confidence interval, which has been repeatedly used in CAT simulation studies with dichotomous items (Chang and Ying, 1996, Chen et al., 2000 , Cheng and Liou, 2000), will be herein applied.

The modified $99 \%$ trait confidence interval of the $n$th step in the CAT implementation, $\Theta_{n}$, is defined as $\Theta_{n}=\left[\hat{\theta}_{n-1}-\delta_{n}, \hat{\theta}_{n-1}+\delta_{n}\right]$, with $\delta_{n}=\frac{c}{\sqrt{n}+1}$, where c is a constant selected according to a coverage probability (Chang and Ying, 1996). As used by Chen et al. (2000), herein $\mathrm{c}=3 . \hat{\theta}_{n}$ is the estimated trait value at step $n$ of the sequential CAT, with $n=2,3, \ldots$, 15 for the fully adaptive and $n=6,7, \ldots, 15$ for the semi-adaptive test. Note that for the fully adaptive test, the first estimated trait value $\hat{\theta}_{2}$ results in a larger confidence interval than its 
counterpart of the semi-adaptive test, $\hat{\theta}_{6}$, whose estimation is based on the response pattern of 5 starting items.

\subsubsection{Data and Methods}

Item pools

The four item pools used in the simulation study are the same as described in 4.4.4, i.e. $I_{300}, I_{600}, J_{300}$ and $J_{600}$

\section{Procedure}

Test length. Since the primary attention is given to the early stage of CAT, the maximum number of selected items was fixed at 15 for all simulations. For the fully adaptive test 15 items were sequentially selected, without replacement, whereas this amount was reduced to 10 for the semi-adaptive test. The initial 5 were concurrently selected by the ISCs described in 4.4.2.

Simulation. Seven distinct simulee's trait levels were investigated, $(\theta=-3,-2,-1,0,1,2,3)$. The response of each simulee was generated in the following manner: The probability of answering each of the $g=3$ categories conditional on the known item parameters and simulee's true trait value $P_{i g \mid \theta_{1}}$, was calculated via (1). Thereafter two thresholds for the cumulative probabilities were determined, $t_{1}$ and $t_{2}$, with $t_{1}=P_{i \mid 1 \theta_{1}}$ and $t_{2}=P_{i|1| \theta_{t}}+P_{i 2 \mid \theta_{t}}$. Based on $t_{1}$ and $t_{2}$, three category intervals were obtained, namely $\left(0, t_{1}\right],\left(t_{1}, t_{2}\right]$ and $\left(t_{2}, 1\right]$. A random number from the uniform distribution $\mathrm{U}(0,1)$ was drawn. The category, i.e. the interval containing the random number was assigned an item-score 1 , and 0 otherwise. For each trait value, 1.000 replications were used for the simulation item responses and the ensuing CAT administration of items.

CAT initialisation. For the full adaptive test the first item was selected by the A-, Doptimality and $\mathrm{KL}$ information criteria over a fixed trait interval $\Theta_{1}=[-3,3]$ and initial true value was assumed to be $\hat{\theta}_{1}=0$. The same is valid for the semi-adaptive test, with the difference that not one but five items were simultaneously selected. Moreover, in the semiadaptive CAT, two further criteria for the selection of the initial $n=5$ items were taken into account: the systematic and the random ISCs (see 4.4.2). 
CAT implementation. The MLE of $\theta$ was computed using the Newton-Raphson method via the Fisher scoring equation for the NRM (see Baker, 1992, p.268-272 for description). Given the estimate $\hat{\theta}_{n-1}$, based on $(n-1)$ administered items, the following $n$th item was selected in a way as to maximise $\Phi_{\mathrm{A}}\left(\ln f_{i}\right)$ in (7), $\Phi_{\mathrm{D}}\left(\operatorname{In} f_{i}\right)$ in (8), and $K_{i}\left(\theta_{0}\right)$ in (9), over the previously described 99\% modified confidence interval (Chen et al., 2000). Once the increment between the provisional estimate $\hat{\theta}_{n}$ and the preceding one $\hat{\theta}_{n-1}$ was too large in absolute terms, i.e. $\left|\hat{\theta}_{n}-\hat{\theta}_{n-1}\right| \geq 4$, the $n$th confidence interval together with $\hat{\theta}_{n}$ assumed the values used in the initialisation. The process stopped at $n=15$ selected items.

Evaluation measures. The ISCs performances were evaluated via the average bias (BIAS) and root mean squared error (RMSE), computed after 1000 replications:

$$
\begin{aligned}
& \operatorname{BIAS}(n, \theta)=\frac{1}{1000} \sum_{1000}\left(\hat{\theta}_{n}-\theta\right) \\
& \operatorname{RMSE}(n, \theta)=\sqrt{\frac{1}{1000} \sum_{1000}\left(\hat{\theta}_{n}-\theta\right)^{2}}
\end{aligned}
$$

The BIAS and RMSE were calculated for the A-, D- optimality criteria and the KL information criterion, for each true trait, $(\theta=-3,-2,-1,0,1,2,3)$, and eventually for each stage of the CAT test with $(n=2,3, \ldots, 15)$ for the full adaptive and $(n=6,7,8, \ldots, 15)$ for the semi-adaptive simulations. In runs where the estimation of $\theta$ quickly degenerated, i.e. for extreme trait values with either fully correct or incorrect response patterns, the BIAS and RMSE were occasionally calculated with less than 1000 replications. This inconvenience, however, was dependent on the quality of the item pool, being often the case for item pool $I_{300}$, seldom for $J_{300}$ and almost absent for $I_{600}$ and $J_{600}$.

\subsubsection{Results}

The RMSEs for the seven trait levels and for the fully and semi-adaptive simulations are displayed in Figures 8 to 15 . Figures 8 and 9 relate to the fully adaptive runs with pools $I_{300}$ and $J_{300}$, respectively. The resulting RMSE of the semi-adaptive tests are displayed in Figures 10 to 13 . For Figures 10 and 11, the simultaneous selection of the 5 initial items took 

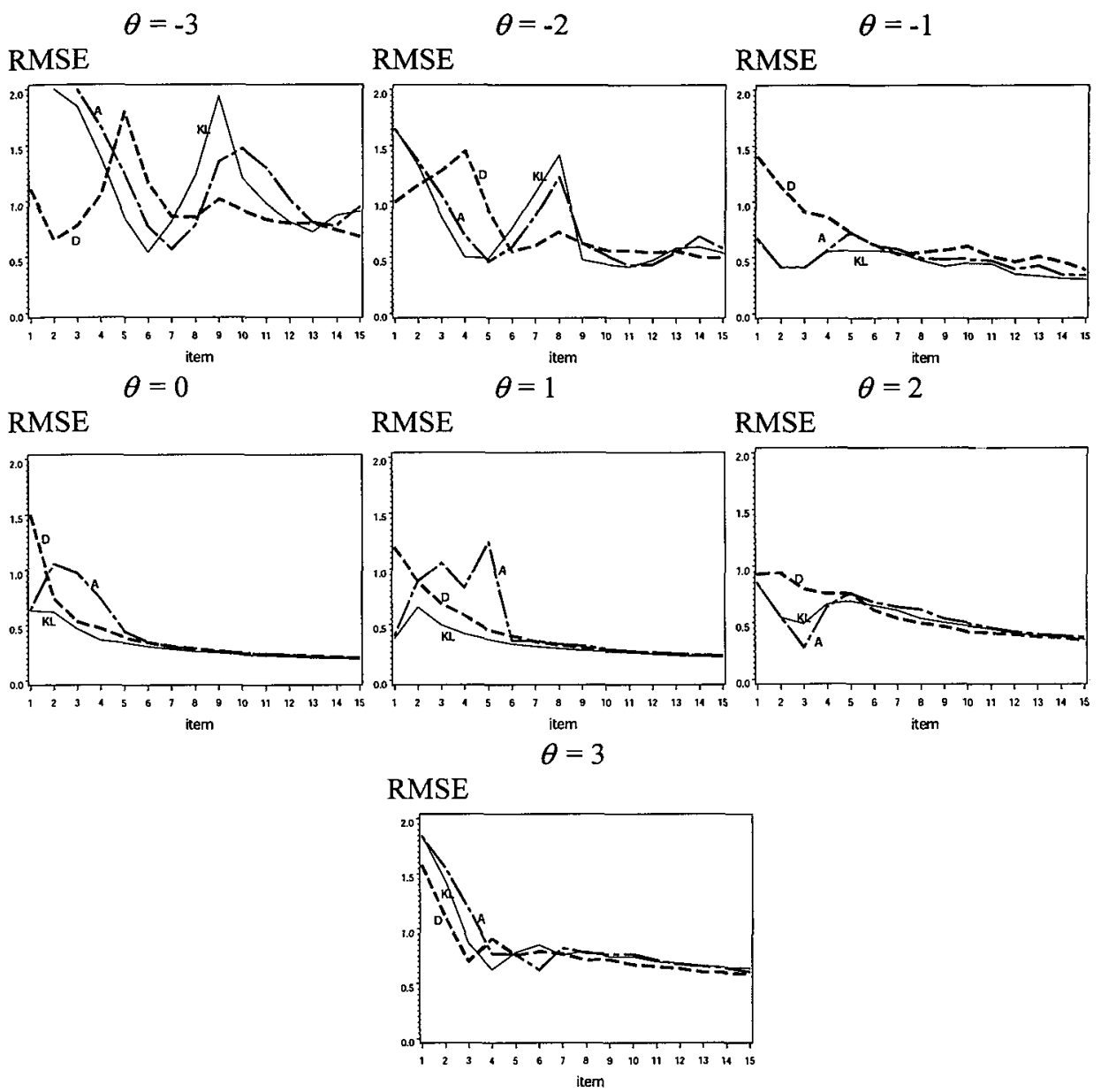

Figure 8. RMSEs for trait estimates for the fully adaptive test (item pool $I_{300}$ ).

place by respective criterion applied in the adaptive selection, i.e. A-, D- or KL. In Figures 12 and 13 the initial 5 items were concurrently selected by the systematic criteria, and thereafter by the $\mathrm{A}, \mathrm{D}$ and $\mathrm{KL}$ criteria adaptively. The results of the simulations with the larger pools, $I_{600}$ and $J_{600}$, resemble to the ones of the smaller pools. For comparative and illustrative purposes, the RMSEs of the fully adaptive tests for $N=600$ are depicted (Figures 14 and 15).

A first inspection of all graphics reveals that for both pools (in all runs), the RMSE fluctuations/values are less evident/smaller for the middle than for the extreme trait values. One could also regard the speed, at which the estimates converge (more quickly for the middle traits), as an indicator for a criterion's performance. Moreover, the RMSE fluctuations 


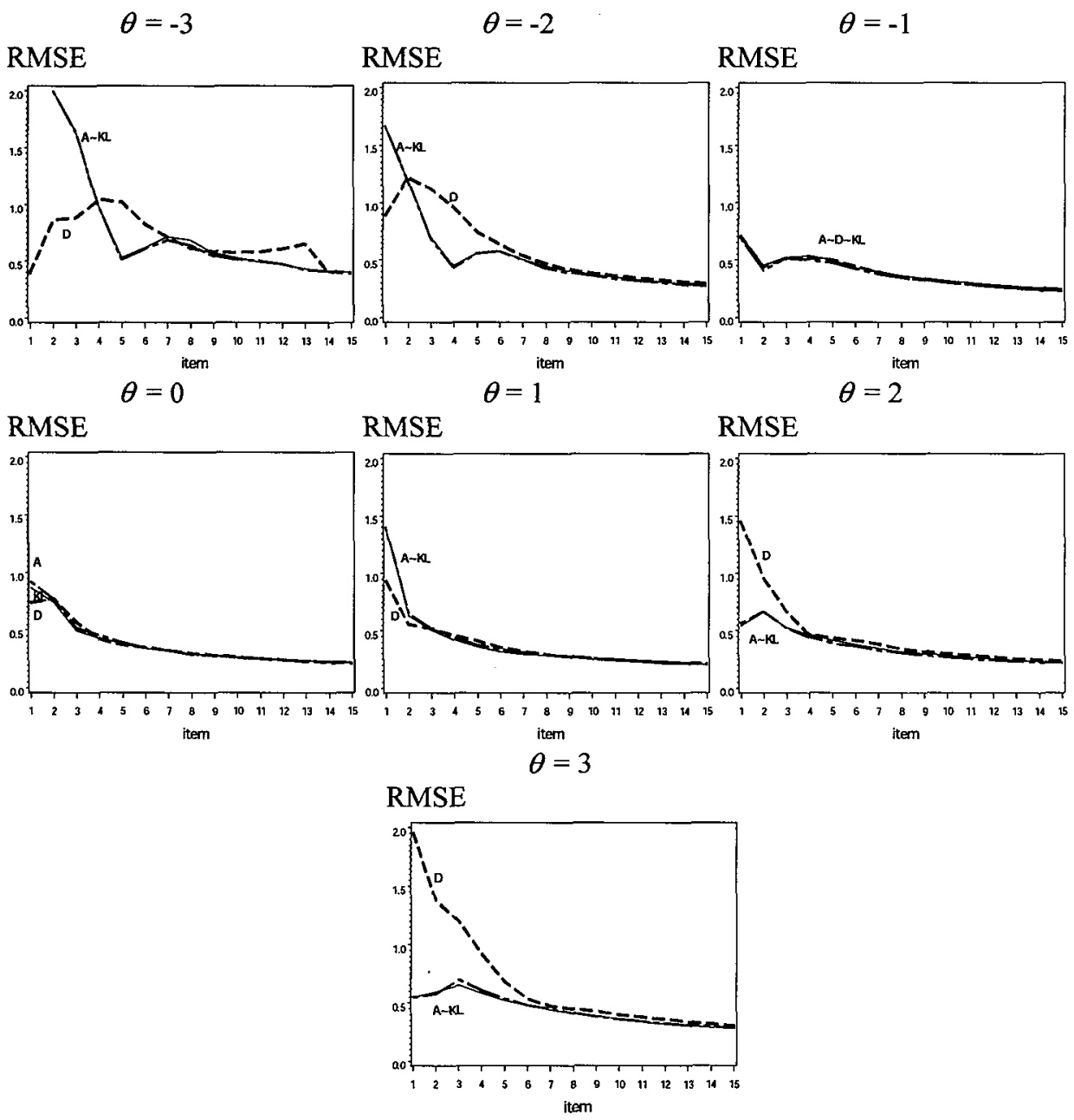

Figure 9. RMSEs for trait estimates for the fully adàptive test (item pool $J_{300}$ ).

are more striking for simulations with $I_{300}$, than $J_{300}$, mainly at the extreme traits. The results for the fully adaptive test with the 600 items pools (Figures 14 and 15) bear strong resemblance with the ones illustrated for $N=300$, with a visible improvement in terms of stability. The magnitude in fluctuations was reduced, or the convergence quicker for the larger pools. Yet, the final efficiency after the administration of 15 items was not significantly changed.

With only a single exception (Figures 10,11 for $\theta=3$ ), the concurrent choice of a small number of items in the semi-adaptive tests (Figures 10 to 13) acted as a RMSE 


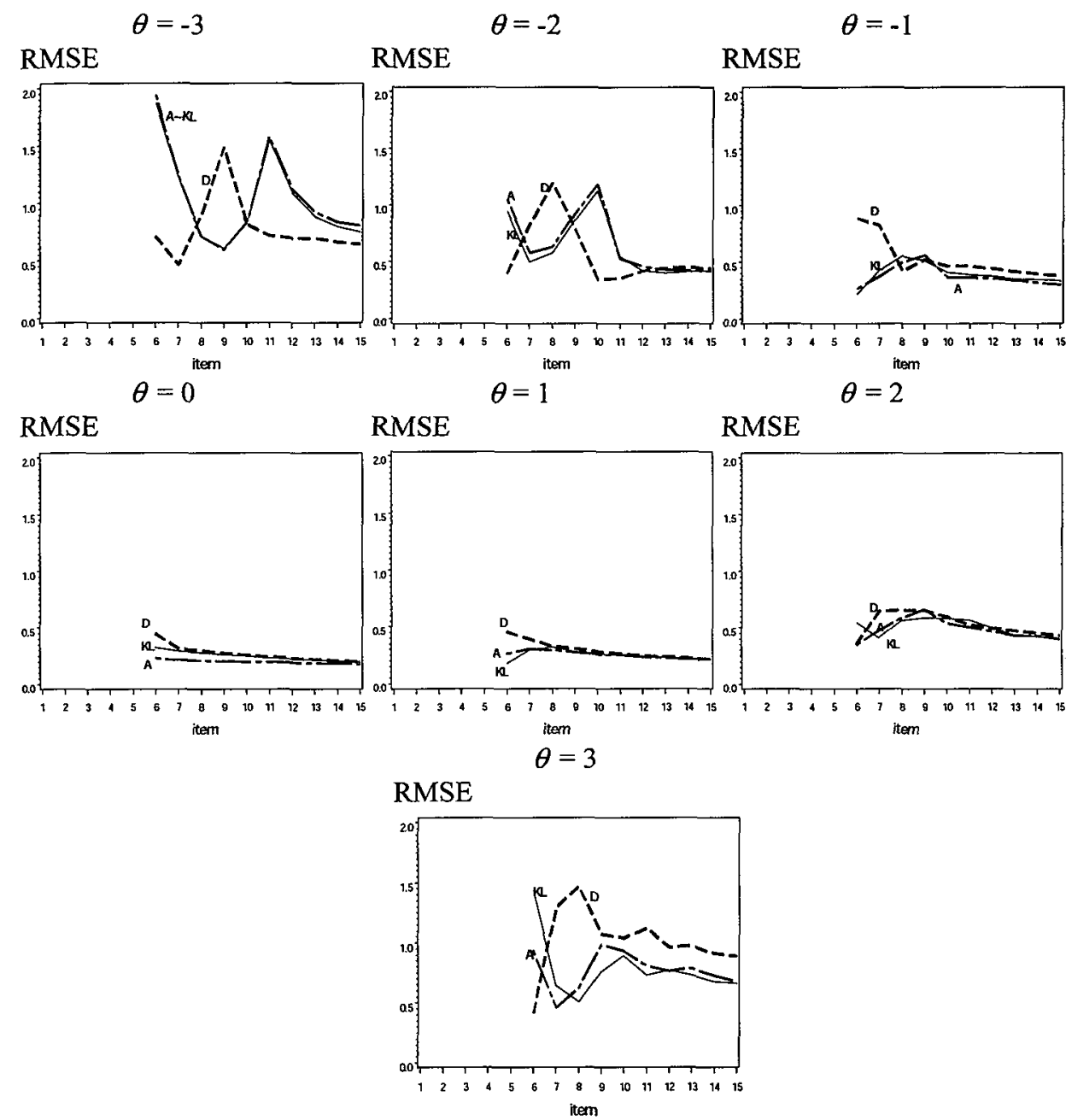

Figure 10. RMSEs for trait estimates for the semi-adaptive test (item pool $I_{300}$ ). Starting items selected by A-,D- and KL criteria.

'stabiliser'. In these runs the final RMSE after 15 items are equivalent to the ones for the fully adaptive situation. However, the fluctuations are considerably smoothed, mostly for the extreme traits. This smoothing was especially striking for those tests, in which the selection of the initial five items took place via the 'systematic' criterion (Figure 12, 13). In the latter case, all three criteria yielded overlapping RMSE curves.

For the fully and semi-adaptive tests, the $\mathrm{A}$ and the $\mathrm{KL}$ criteria display similar RMSE lines (parallel or, in the runs with pool $J_{300}$, overlapping). In general, and mainly for pool $I_{300}$ 


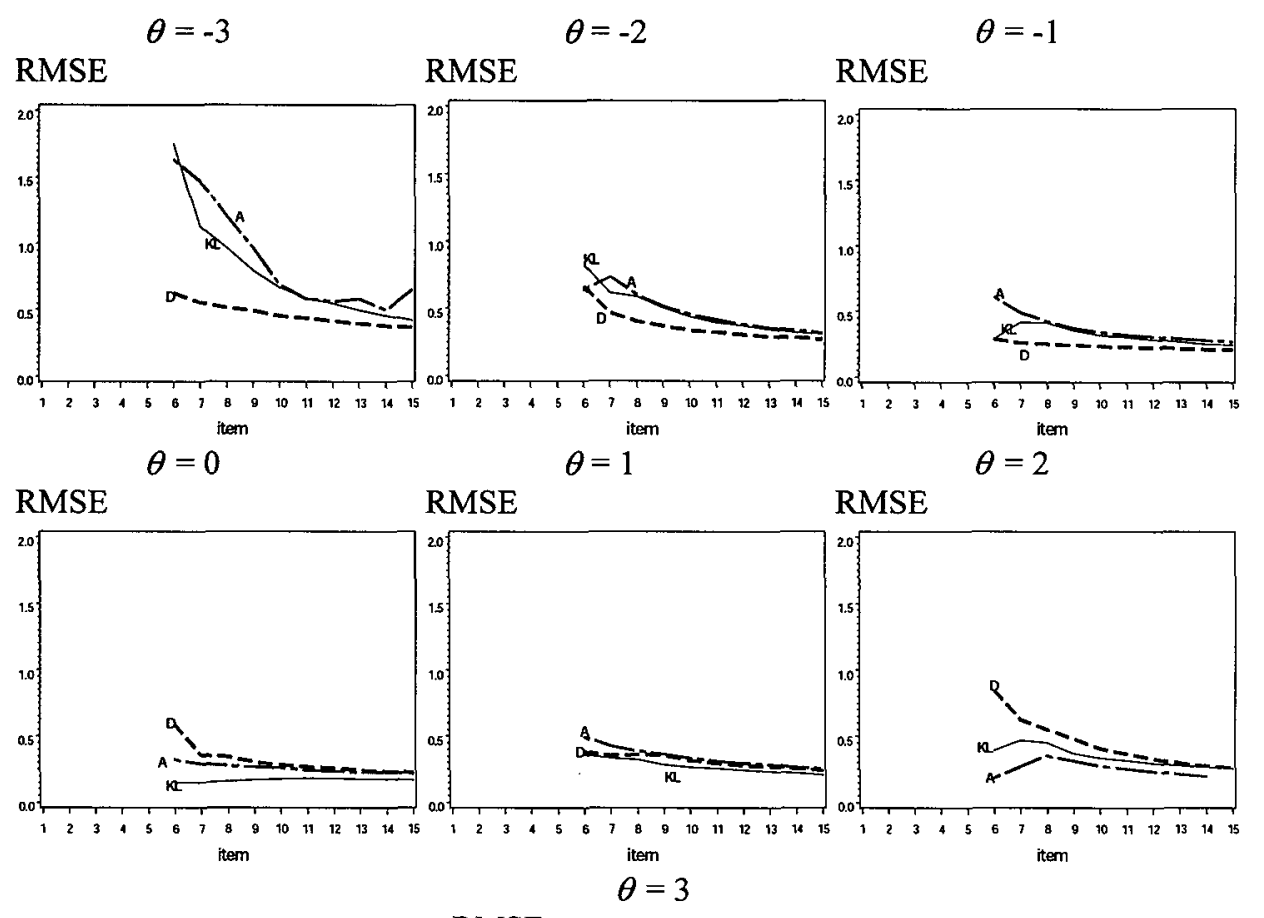

\section{RMSE}

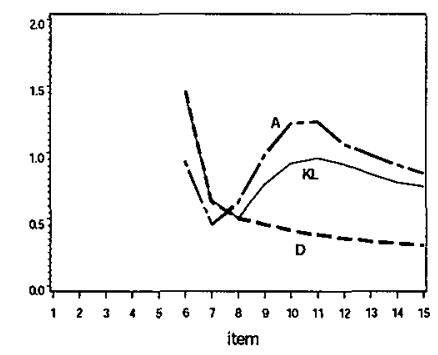

Figure 11. RMSEs for trait estimates for the semi-adaptive test (item pool $J_{300}$ ). Starting items selected by A-,D- and KL criteria.

(Figure 8), the A-criterion is marginally less stable and/or less efficient after the administration of 15 items than the $\mathrm{KL}$ criterion. Both criteria did rather well for central trait values, for which abundant information is available. For the more extreme traits, however, their RSME lines were rather instable. This stands in contrast to the D-optimality criterion that displayed comparatively smother RSME behaviour across all trait values (see above all runs with pool $I_{600}$ and $J_{600}-$ Figures 14 to 15 ). Note the fairly monotone course of the RMSE 

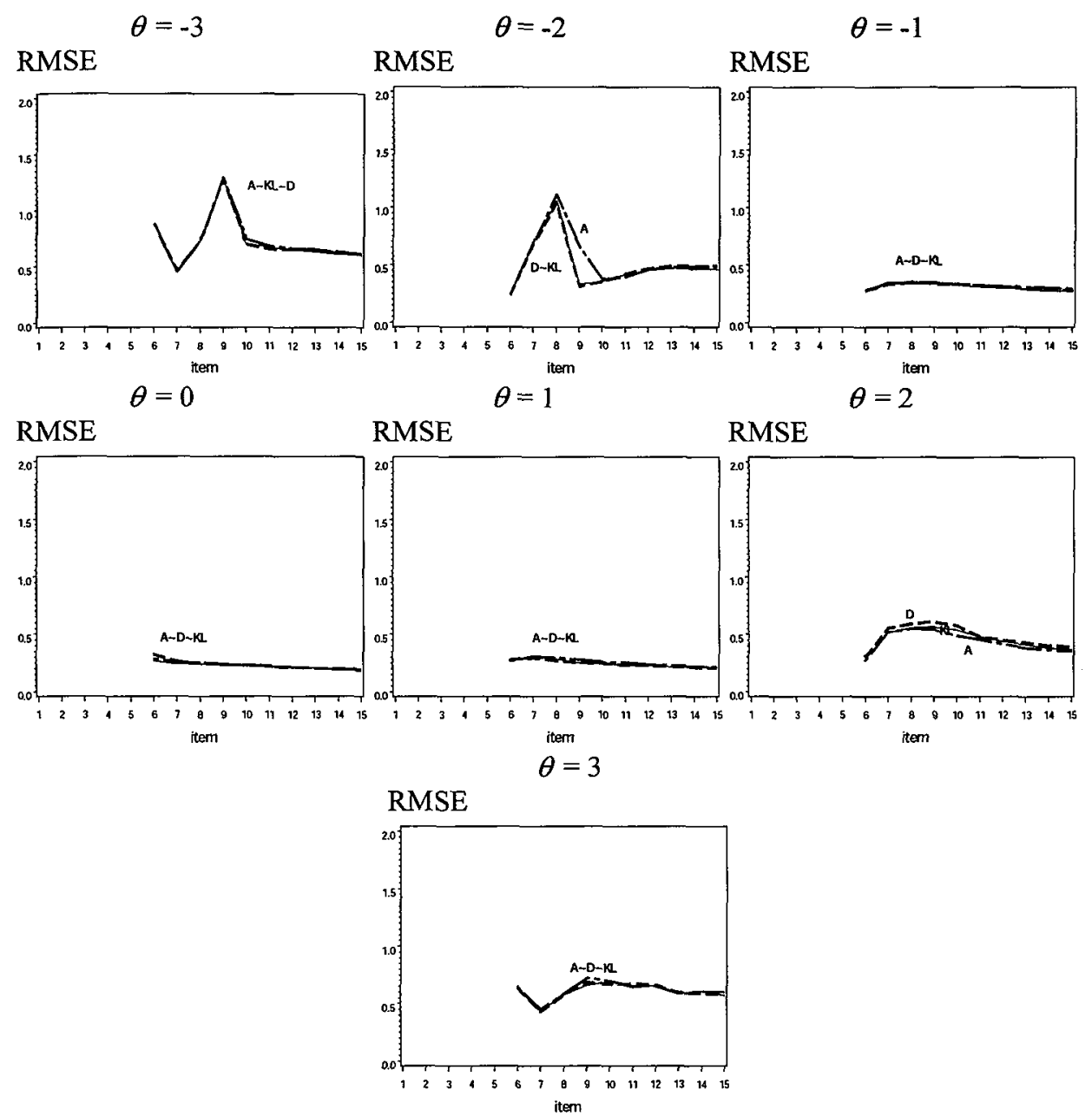

Figure 12. RMSEs for trait estimates for the semi-adaptive test (item pool $I_{300}$ ). Starting items selected by the ' $s$ ' criterion.

towards the asymptote (exception again in Figure 10 for $\theta=3$ ). However, contrary to the A and KL criteria, the D-criterion was efficient for $\theta=3$ in Figure 11.

The BIAS results are omitted, because they follow the same pattern as the RMSE. However, one aspect is worth mentioning. MSE gradually decreases as more items are administered. MSE is equal the sum of BIAS squared plus the variance, i.e. MSE $=B I A S^{2}+$ variance. At the beginning of the items administration, the MSE values can be traced back almost exclusively to the BIAS and very little to the variance, mainly for low and high trait values. This picture reverses towards the end of the 15 items, along which the BIAS 


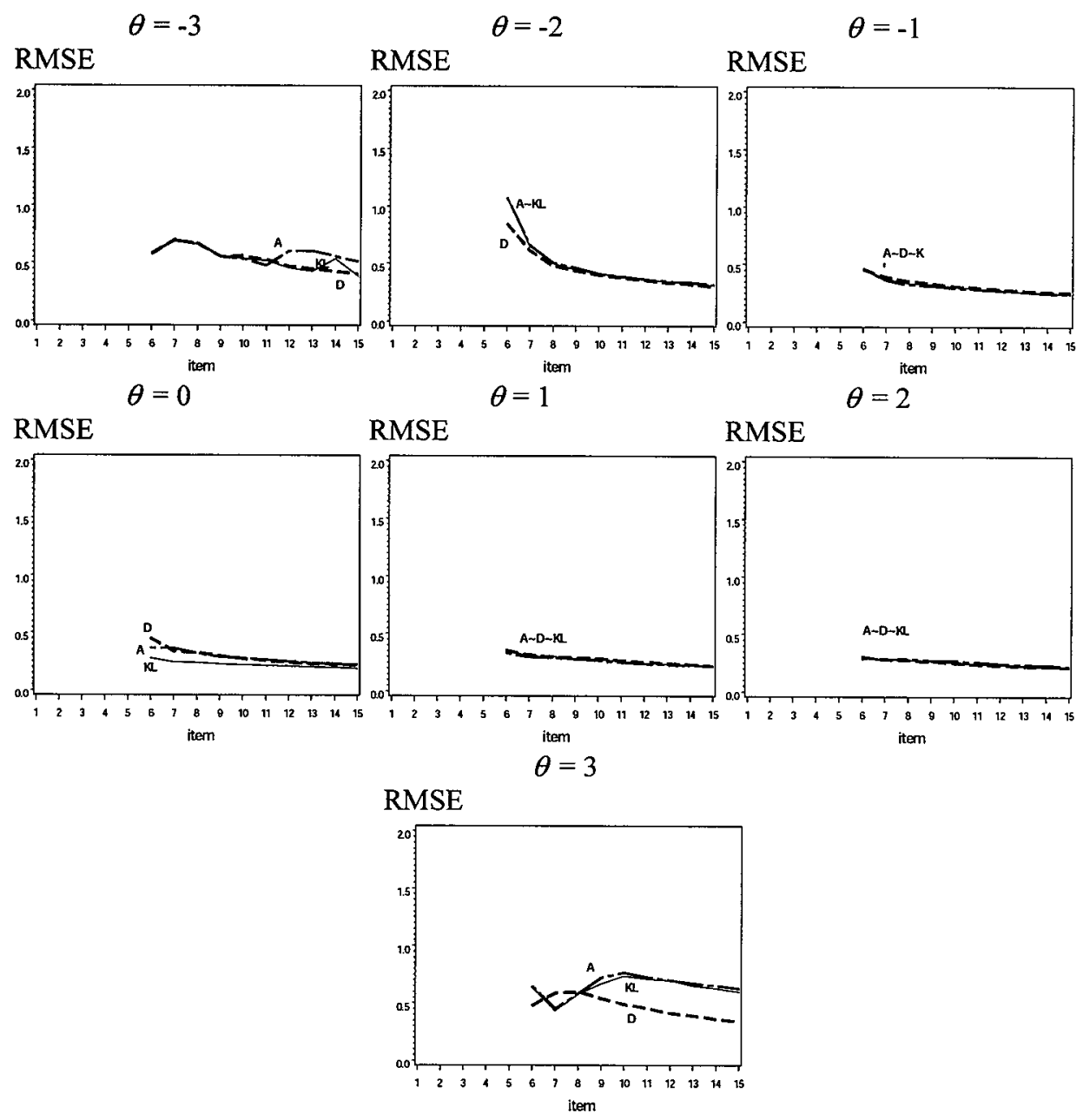

Figure 13. RMSEs for trait estimates for the semi-adaptive test (item pool $J_{300}$ ). Starting items selected by the ' $s$ ' criterion.

component of MSE reduces and the variance's part increases. For example, the RMSE values for $\theta=-3$ in the fully adaptive run $\left(I_{300}\right)$ with the A-criterion are, for the $1^{\text {st }}$ and $15^{\text {th }}$ items respectively, 2.647 and 0.952. From those, the BIAS components are 2.647 and -0.0197 . Under the same conditions, yet for $\theta=0$ the relation RMSE/BIAS becomes $0.637 / 0.001$ and $0.200 / 0.002$ for the $1^{\text {st }}$ and $15^{\text {th }}$ items, respectively. 


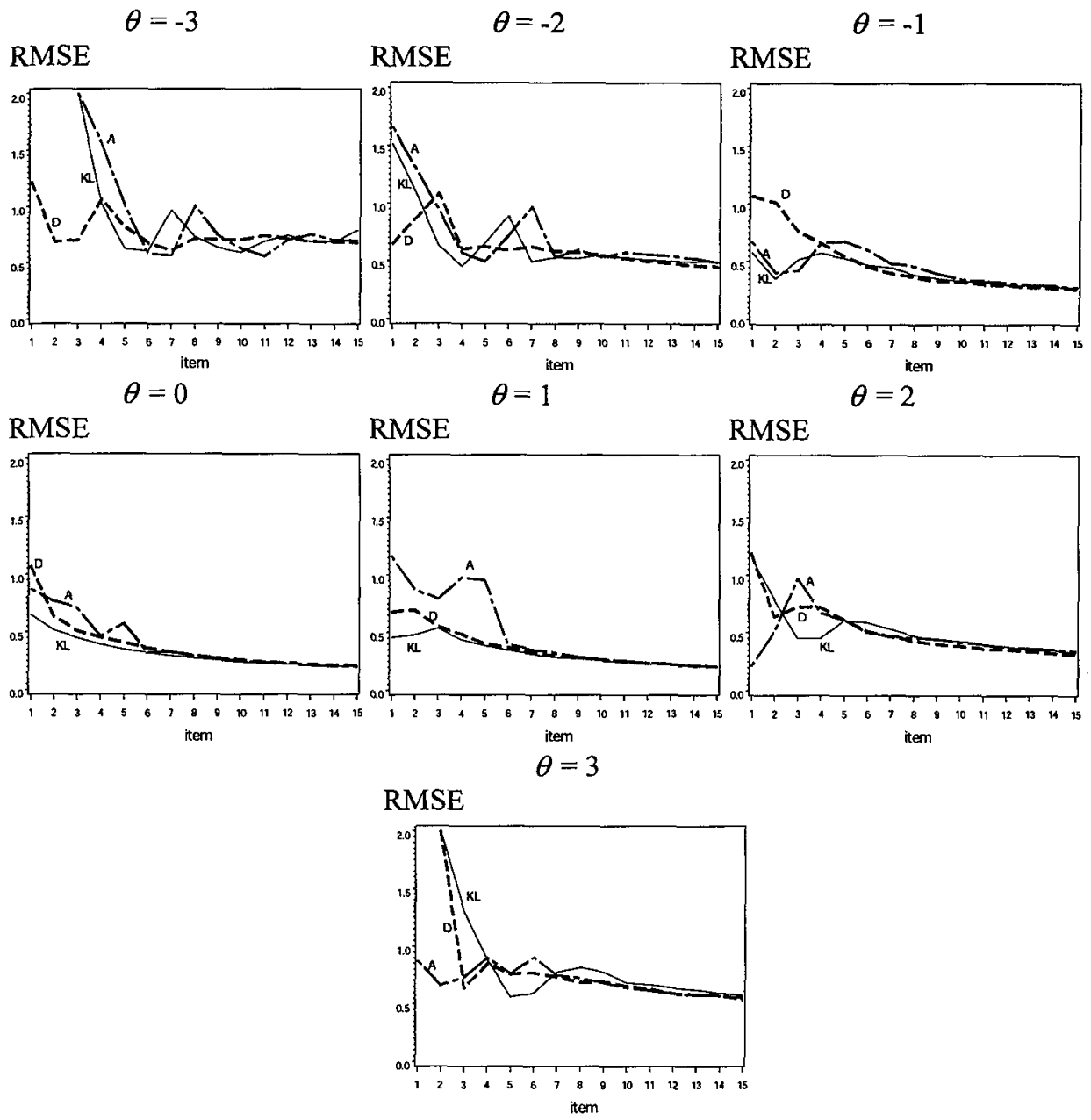

Figure 14. RMSEs for trait estimates for the fully adaptive test (item pool $I_{600}$ ).

\subsection{Discussion}

On the item pool effect. The centrifugal deterioration of all criteria RMSE (rather stable and efficient at the middle trait values, worsening towards the extremes) is a direct reflection of the information availability. Both pools $I_{300}$ and $J_{300}$ provide more information around central traits and less towards the range extremes, with a slight shift to the right, towards higher traits. This discrepancy in the information dispersion is more accentuated for pool $I_{300}\left(I_{600}\right)$ with relatively little information at the low and high trait levels (see Figure 1). Consequently, the 

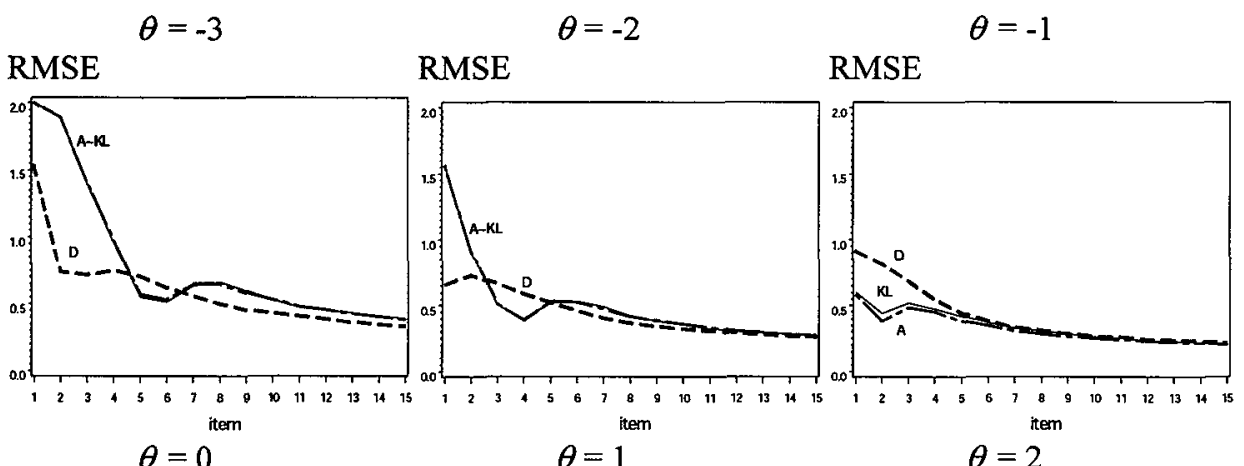

\section{RMSE}

$\theta=0$

$\theta=1$

RMSE

RMSE
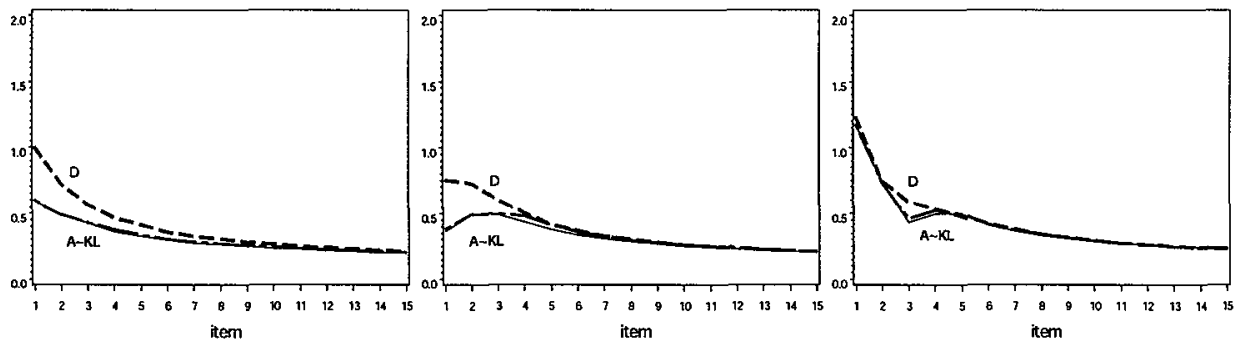

$\theta=3$

\section{RMSE}

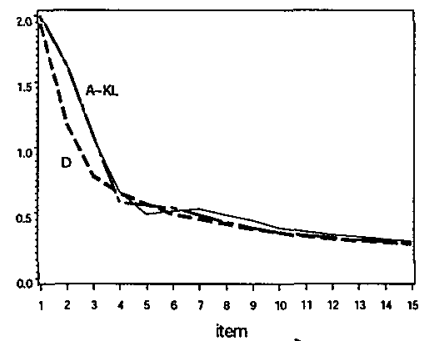

Figure 15. RMSEs for trait estimates for the fully adaptive test (item pool $J_{600}$ ).

RMSE fluctuations are more evident for simulations with $I_{300}$, than $J_{300}$, mainly at the extreme traits. Hence, regardless of the premise (fully or semi-adaptive), the information availability emerges as the major factor influencing the performance of an ISC. In general, the more uniform the coverage, the less instable and the more efficient the estimation. This is valid to all applied criteria.

The slight performance improvement for the runs with larger pools $(N=600)$ raises the question whether it is worth working with larger item banks envisaging more efficient estimates. One should bear in mind that larger banks considerably increase the CPU time. 
On the semi-adaptive test. The overlapping RMSE lines of the 'systematic' semi-adaptive test suggests that given the same robust starting constellation of items, the different criteria tend to coincide in their selection. The criteria differ in their modus operandi. However, they converge in their selection once the trait interval is not only equal, but above all narrow. These premises are given by the 'systematic' item selection. In accordance with the results in part 1 , this outcome pinpoints the relative efficiency as a more reliable indicator for the criterion's choice in the fixed part of the test, instead of the absolute information, as originally presented (see Tables 1 and 2).

On the ISC. In the matter of stability and efficiency of trait estimation, this study shows that the role of the ISC is secondary to the pool information quality. Ultimately, a criterion cannot extract information, from where there is little available. Among the three applied criteria, the D-criterion did not come to the fore as more efficient. Yet, its relative stability across diverse traits lends support the hypothesis that it could be an effective tool to counteract the attenuation problem in the instable phase of CAT.

\subsection{Concluding remarks}

An item pool with a uniform information dispersion offers the best grounds for a CAT setting, irrespective of the criteria. In practice, however, item banks normally have a bellshaped information curve, with relatively poor information at extreme traits. In this case, the issue of a criterion's choice becomes more pertinent.

The FI point maximum information criterion, the maximum interval information criterion (i.e. the A-optimality criterion) and to a lesser extent the KL-information criterion, are known for targeting items with high discrimination parameters. The key aspect of highly discriminative items is their high trait-specificity. They are very informative for a narrow range of trait values and, therefore, of little use for the remaining traits. This feature is not necessarily advantageous for the CAT early stage, when the trait estimation is uncertain. In this phase, the selection of such items can backfire, generating instability due to the enhanced risk of the attenuation problem.

Van Rijn et al. (2002) claim that in the beginning of a CAT, items that give information over a range of trait values are desirable. The results presented in this paper are consistent with this assumption at least in two different ways.

Firstly, the semi-adaptive approach with the 'systematic' item selection stabilised the trait estimation appreciably. Not surprisingly, this selection happened to provide broader 
information coverage for the targeted traits. Secondly, a similar smoothing effect, though in lesser degree, was obtained by the initial selection of low discriminative items by the Doptimality criterion. Contrary to the highly discriminative items, the low ones offer little but some information for a wider range of traits. This wider coverage is the most likely reason behind the D-criterion's robust performance. All in all, these findings reinforce the argument that wider and not necessarily higher information is beneficial for the early stage estimation.

This work demonstrates the viability of the D-optimality ISC for CAT. This criterion targets items with low discrimination parameters for large trait intervals. On the other hand, it switches to discriminative items, once the interval becomes narrow. This fine-tuning to the underlying trait distribution makes the $\mathrm{D}$-criterion tailor-made for adaptive tests, which have the trait estimates and intervals constantly updated. Furthermore, this adjustment acts analogously to an $a$-Stratified CAT (Chang and Ying, 1999), in which items with the low discrimination parameters are selected first, followed by the selection of high discrimination items. The difference, however, is that this self-regulating mechanism is intrinsic to the Dcriterion, requiring no previous stratification of the pool. Based on these findings one can anticipate the D-criterion flexibility to be rather advantageous in the handling of the item exposure problem. 


\section{D-optimality item selection criterion in CAT early stage - A study with the Graded Response Model}

This chapter has been submitted for publication with co-authors M.P.F. Berger and F.E.S. Tan.

Acknowledgments: We wish to thank Michiel Hol and Harrie Vorst for kindly allowing us to use item parameters from the 'School Attitude Questionnaire'. 


\title{
The D-optimality item selection criterion in CAT early stage - A study with the Graded Response Model
}

\begin{abstract}
During CAT early stage, item selection criteria (ISC) based on Fisher's Information (FI) often produce less stable estimates than the Kullback-Leibler (KL) global information criterion. Robustness against early stage instability has been also reported for the D-optimality criterion in a polytomous CAT with the Nominal Response Model. The current paper shows the Dcriterion's robustness to be reproducible for the Graded Response Model (GRM). For comparative purposes, the A-optimality and the KL-criteria are applied. The sequential selection of items for each criterion is closely investigated as a function of test progression and item bank composition. The obtained results shed some light on how the selection of specific item parameters underlies the criteria performances evaluated via accuracy and precision of trait estimation. Additionally, the criteria item exposure rates are computed and compared, without the use of any exposure controlling measure. On the account of early stage stability, measurement precision and accuracy, numerical simplicity and, less evidently, item exposure rate, the $\mathrm{D}$-optimality criterion can be safely recommended for the selection of items in adaptive testing.
\end{abstract}

Keywords: Item selection criterion, D-optimality criterion, CAT early stage, Graded Response Model, item exposure rates. 


\section{The D-optimality item selection criterion in CAT early stage - A study with the Graded Response Model}

\subsection{Introduction}

Literature references on the subject of item selection criteria (ISC) in computerised adaptive testing (CAT) are plenty. They mirror past and recent efforts concentrated on the formalisation, operationalisation and comparison of ISCs in an attempt to find those that yield accurate (unbiased) trait estimates, preferably with low expenditure in terms of number of administered items (Chang and Ying, 1996; Veerkamp and Berger, 1997; van der Linden, 1998; Eggen, 1999; Chen et al., 2000; Cheng and Liou, 2000; van der Linden and Pashley, 2000; van Rijn et al., 2002; Weissman, 2003; Lima Passos et al. 2004).

Concurrently, much attention has been directed to the handling of the item exposure problem (Chang and Ying, 1999; Chang et al., 2001; Cheng and Liou, 2003; Chang and van der Linden, 2003, Yi and Chang, 2003). The latter relates to the question of how to make a balanced use of items in a pool aiming at a uniform frequency of items administration. For this purpose it is crucial to apply controlling measures on item selection procedures. This is done mainly for test security reasons in an attempt to avoid items' overexposure.

Reconciling effective item pool usage with accurate trait estimation is a difficult task. These two objectives have antagonistic effects: making a balanced use of available items in a pool results in little exposure problem, yet generally at expense of precision and accuracy of trait estimation (Cheng and Liou, 2003). The opposite applies, i.e. using specific criteria to maximise information on a trait leads to overexposure of the most informative items. Precision and accuracy of estimation are usually measured via the estimator's mean squared error (MSE), alternatively root mean squared error (RMSE) and bias (Van Rijn, P.W. et al., 2002).

ISCs based on the maximisation of Fisher's information (FI), either as a point or interval information criterion (Berger and Veerkamp, 1996) seem to be particularly affected by the problem of unbalanced exposure (see Cheng and Liou, 2003, Chang and van der Linden, 2003). This problem is commonly associated with the selection of the most discriminative items. The choice of those items has been additionally regarded as the source of the so-called CAT early stage instability. Van der Linden and Pashley (2000, p. 7) distinguish three stages of trait estimation in adaptive testing - initial, interim and final trait estimation - each one with its requirements and problems. The initial or early stage refers to the phase of trait estimation after the administration of only a few items. It is well known that 
during this phase trait estimates need to be corrected for bias. Furthermore, this test stage is also characterised by its propensity to instability. The latter is often expressed in form of RMSE and bias fluctuations along the sequential course of the test. At the early stage, the trait estimator $\hat{\theta}$ might strongly deviate from the true trait value $\theta$. Selecting a highly discriminative item to match $\hat{\theta}$ can have the downside effect of providing little information on the true trait $\theta$. As a result, the $\hat{\theta}$ 's sequential convergence to the true value, $\theta$, can be considerably delayed. This problem is known as the attenuation paradox (van der Linden and Pashley, 2000) and is the likely reason behind early stage instability.

Lima Passos et al. (2004) applied an ISC based on the product, instead of a sum of FI measures over a trait interval: the D-optimality criterion. Their study was carried out in a polytomous CAT with the Nominal Response Model. It demonstrates that the D-criterion targets items with relative low discrimination parameters at the test beginning, switching to more discriminative items, as the sequential test unfolds, displaying thus greater flexibility of items choice. In so doing, it often produced more stable estimates than other ISCs, with comparable accuracy and precision. The item exposure question had not been specifically tackled, but it has been cogitated that the D-criterion would possibly allow for a more effective use of items in the pool.

With the introduction of the Kullback-Leibler (KL), Information criterion to an adaptive test setting (Chang and Ying, 1996), a useful tool against early stage instability became available. However, a study by Fan and Hsu (1996) indicated that this criterion's robustness should not be taken at its face value, being dependent on the size/quality of item pool. The study of Lima Passos et al. (2004) partially endorsed KL-information' general robustness. So far there is no literature reference reporting the effects of the Kullback-Leibler Information criterion on the item exposure problem.

Objective of the current paper is to compare the selective strategies of three ISCs, the A- and D-optimality criteria, both based on Fisher's Information, and the KL-criterion. By 'selective strategies' is meant the specific choice of items with respect to their parametric properties as a function of the test progression. Focus is centred on the shifting of these properties along the test. The main task is to associate these changes with the attained criteria performances, measured by the resulting root mean squared error (RMSE) and bias of estimator. At last, the criteria will be also evaluated with respect to the item exposure rates. No specific measure for controlling the item exposure, as known from the literature (Chang and Ying, 1999; Chang et al., 2001; Cheng and Liou, 2003; Chang and van der Linden, 2003, 
Yi and Chang, 2003) is applied. For this investigation a CAT simulation study is carried out with polytomous items described by the Graded Response Model (GRM).

The paper is structured as follows: A concise description the Graded Response Model (GRM) ensues together with the definitions of the FI and $\mathrm{KL}$ information measures (section 2), upon which the applied ISCs are based. These are described at greater length in section 3. Section 4 outlines the steps constituting simulation study, whose results are displayed in section 5 . The final section summarises the obtained insights and provides a few guidelines for test-makers.

\subsection{The Graded Response Model and information measures}

\subsubsection{The Graded Response Model}

The Graded Response Model (GRM), introduced by Samejima (1969), belongs to the class of models, for which the responses are measured on an ordinal scale. The response options are ordered with respect to the degree of success to the solution of a problem. The ordering of the response options is a necessary precondition to be met in order to enable estimation of the cumulative probability response functions $P_{i g \mid \theta}^{*}$, individually. The equation that describes a boundary curve is given by:

$$
P_{i g \mid \theta_{i}}^{*}=\frac{\exp \left[a_{i}\left(\theta_{t}-b_{i g}\right)\right]}{1+\exp \left[\left(a_{i}\left(\theta_{t}-b_{i g}\right)\right]\right.}
$$

where $\theta_{t}$ is the latent trait variable of examinee $t$, with $t=1,2, \ldots, T, a_{i}$ is the discrimination parameter of item $i$, for all the boundary curves $P_{i g \mid \theta_{i}}^{*}$, with $i=1,2, \ldots, n, b_{i g}$ is the location parameter associated with boundary curve $g$ of item $i$ with $g=1,2, \ldots, k_{i}$ and $k_{i}$ represents the total number of response categories. To maintain the underlying order of response categories, the $\left(k_{i}-1\right)$ location parameters need to be ordered, $b_{i\left(k_{i}-1\right)}<\ldots<b_{i g}<\ldots b_{i 1}$.

The probability of an examinee selecting an item response category conditional on his/her $\theta$-level, $P_{i g \mid \theta_{t}}$ can be expressed in terms of boundary probabilities $P_{i g \mid \theta_{i}}^{*}$. The probability of the response to fall in category $g$ is given by the difference between any two adjacent boundaries (Baker, 1996, p.227), i.e.: 


$$
P_{i g \mid \theta_{t}}=P_{i(g-j) \mid \theta_{t}}^{*}-P_{i g \theta_{t}}^{*}
$$

The boundary $P_{i g \theta_{i}}^{*}$ curves are interpreted as the probability of a response belonging to a category greater than $g$. For an item with four response alternatives, i.e. $k_{i}=4$, for instance, $P_{i z \mid \theta}^{*}$ represents the probability that an examinee will select category 3 or 4 . Similarly, the probability that an examinee will select categories $g=1,2,3$ or 4 is $P_{i|0| \theta_{l}}^{*}=1$ and a category larger than $k_{i}=4$ is $P_{i k_{i} \mid \theta_{t}}^{*}=0$. Note that because the boundary curves are cumulative probabilities of the response categories with $\sum_{g=1}^{k_{1}} P_{i g \mid \theta_{t}}=1$, there is always one less location parameter $b_{i g}$, than there are item response categories, i.e. there are $\left(k_{i}-1\right)$ boundaries for $k_{i}$ item categories. Thus, $\left(k_{i}-1\right)$ values of location parameters of the boundary curves $b_{i g}$ are used to describe the location of $k_{i}$ response categories, denoted $b_{i g}^{\prime}$. For the first and last monotone response categories, $b_{i 1}^{\tilde{1}}=b_{i 1}$ and $b_{i \tilde{k}_{i}}=b_{i\left(k_{i}-1\right)}$. For all other response options $b_{i g}^{\tilde{g}}=$ $\left(b_{i g}+b_{i(g-1)}\right) / 2$ (Samejima, 1969, p. 34, Baker, 1992, p. 228). The latter locates the modal point of the non-monotone item response category characteristic curve (IRCCC). In general all $b_{i g}^{\sim}$ parameters are used to describe the location of an item response category on the latent trait scale, analogous to that used with dichotomously scored items (Baker, 1992, p. 229).

\subsubsection{Information measures}

There are basically two major information measures applied in test design optimisation known as Fisher's and Kullback-Leibler Information.

Fisher's Information. For the GRM, a single item discrimination parameter $\mathrm{a}_{\mathrm{i}}$ is shared by all cumulative boundary curves. The total Fisher's information (FI) of an item on $\theta$ can also be defined with respect to the boundary probabilities, so that,

$$
\operatorname{Inf}_{i . \theta_{t}}=\sum_{g} \frac{a_{i}^{2}\left(w_{g-1}-w_{g}\right)^{2}}{P_{i(g-l) \mid l \theta_{t}}^{*}-P_{i g \mid \theta_{t}}^{*}}
$$

where $w_{g}=P_{i g \mid \theta_{t}}^{*}\left(1-P_{i g \theta_{t}}^{*}\right)($ Baker, 1996, p. 245-246). 
Kullback-Leibler Information. Chang and Ying (1996) were the first to suggest an ISC based on Kullback-Leibler (KL) information. KL information function of an item $i$ is based on a ratio between likelihoods of two different trait values, $\theta$ and $\theta$, and measures the distance between them. The larger this value, the easier it is to discriminate between the trait values. For a polytomous IRT model the KL Information for a single item $\mathrm{i}$ can be expressed as:

$$
K_{i}\left(\theta, \theta_{0}\right)=\sum_{g} P_{i g \mid \theta_{o}} \log \left(\frac{P_{i g \mid \theta_{0}}}{P_{i g \mid \theta}}\right) .
$$

These two information measures are the building stones of the selection criteria described in the ensuing session.

\subsection{Item selection criteria (ISC)}

Three ISCs will be compared, namely the A- and D-optimality criteria, which are objective functions $\Phi($.) of Fisher's information, and a criterion based on KL- information.

In test design optimisation the function $\Phi$ needs to be maximised along a latent trait interval $\Theta=\left[\theta_{\mathrm{L}}, \theta_{\mathrm{U}}\right]$, with $\theta_{t} \in \Theta, t=1,2, \ldots, T$. Thus $t$ is the index that runs through the elements $\theta_{t} \in \Theta$. $\theta_{\mathrm{L}}$ and $\theta_{U}$ represent the interval's lower and upper boundaries, with index $t=1$ and $t=T$, respectively. The A-optimality, trace or sum criterion, is given by:

$$
\Phi_{\mathrm{A}}\left(\operatorname{Inf} f_{i, \theta_{t}}\right)=\sum_{t=I}^{T} \operatorname{Inf} f_{i, \theta_{t}}
$$

where $\operatorname{Inf}_{l, \theta_{t}}$ is Fisher's information of item $i$ on a single $\theta_{l}$, as defined in equation (3). The Aoptimality criterion is a sum of information of an item $i$ over traits $\theta_{l}$. It should be noted that, the A-optimality criterion is a discrete version of the area under the curve of the item information function over $\Theta$. In this sense, the A-criterion is equivalent to the maximum interval criterion, as proposed by Veerkamp and Berger (1997), where $\Theta$ is a confidence interval of the estimator $\hat{\theta}$.

The product, determinant or D-optimality criterion is defined as: 


$$
\Phi_{\mathrm{D}}\left(\operatorname{Inf}_{i, \theta_{i}}\right)=\prod_{t=1}^{T} \operatorname{Inf}_{i, \theta_{i}} .
$$

To obtain numerical stability and facilitate computational work, the objective function in (6) can be replaced by $\Phi_{\mathrm{D}}\left(\operatorname{In} f_{i, \theta_{t}}\right)=\sum_{i=l}^{T} \log \operatorname{Inf} f_{i, \theta_{i}}$.

The feasibility of the D-optimality criterion for CAT with the Nominal Response Model has been shown by Lima Passos et al. (2004). Their findings pointed to a general robustness of this ISC against the early stage instability.

For more detailed descriptions of the optimality criteria in a general context of design optimisation, see Atkinson and Donev (1992). In the specific domain of optimal test design, Berger and Veerkamp (1996) provide an overview of different objective functions $\Phi$ based on FI, including the A- and D- criteria. Further references related to optimal assemblies for fixedform tests with the A- and D-optimality criteria are given by Berger and van der Linden (1995), Berger and Mathijssen (1997), Berger (1998) and Lima Passos et al (2004).

An alternative to criteria defined on $\mathrm{FI}$ is one based on the global or $\mathrm{KL}$ information. Chang and Ying (1996) proposed an ISC that integrates the KL function $K_{i}\left(\theta, \theta_{0}\right)$ in (4) over a given interval $\Theta=\left[\theta_{\mathrm{L}}, \theta_{\mathrm{U}}\right]$, where the true trait value $\theta_{0}$ is fixed and known with $\theta_{0} \in \Theta$ :

$$
K_{i}\left(\theta_{0}\right)=\int_{\theta_{L}}^{\theta_{U}} K_{i}\left(\theta, \theta_{0}\right) d \theta
$$

The area under the curve of the KL function associated with the trait interval $\Theta$ represents the average information index to be maximised. The measure $K_{i}\left(\theta_{0}\right)$ indicates how powerful a specific item is in holding other trait values from the true one apart.

\subsection{The simulation study}

\subsubsection{Data and methods}

Item pools

Two pools were used in the simulation study, $I_{300}$ and $I_{600}$ containing $N=300$ and 600 items, respectively. The items' discrimination and location parameters have been randomly 
generated taking the parameters of 48 three categories $(g=3)$ items described by the GRM as a blue print. These calibrated items stem from a subscale of the so-called 'School Attitude Questionnaire', administered to high school students in the Netherlands, which specifically measures their motivation concerning work at school (Vorst, 2000).

For item parameters generation both mean and variance-covariance structure of the original 48 items were incorporated into a multivariate normal distribution. Histograms of the parameters distributions are given in Figure 1 for pools $I_{300}$ and $I_{600}$ together with their item bank information functions, Figure 2, computed as the sum of all item information functions. It can be seen that the item banks provide more information for the lower-middle range of the latent trait scale, and relatively poor information for the higher trait levels. It is assumed that the two item banks are realistic examples of pools for the GRM.

\section{Procedure}

Test length. Since primary attention is given to the early stage of CAT, the maximum number of selected items was 15 for all simulations. They were sequentially selected, without replacement within one test, not over replications.

Response Generation. Seven distinct simulee's trait levels were investigated, $\theta=(-3,-2,-1,0$, $1,2,3)$. The response of each simulee was generated in the following manner: The probability of answering each of the $g=3$ categories conditional on the known item parameters and simulee's true trait value $P_{i g \mid \theta_{t}}$, was calculated via equation (1) and (2). Thereafter two thresholds for the cumulative probabilities were determined, $t_{1}$ and $t_{2}$, with $t_{1}=P_{i 1 \mid \theta_{t}}$ and $t_{2}=$ $P_{i \mid \theta_{t}}+P_{i 2 \mid \theta_{t}}$. Based on $t_{1}$ and $t_{2}$, three category intervals were obtained, namely $\left(0, t_{1}\right],\left(t_{1}, t_{2}\right]$ and $\left(t_{2}, 1\right]$. A random number from the uniform distribution $\mathrm{U}(0,1)$ was drawn. The category, i.e. the interval containing the random number was assigned an item-score 1, and 0 otherwise. For each trait value, maximal 1.000 replications were used for the simulation item responses and the ensuing CAT administration of items.

CAT initialisation. The first item was selected by the A-, D- optimality and KL information criteria over a fixed trait interval $\Theta_{1}=[-3,3]$ and the initial trait value was assumed to be zero, $\hat{\theta}_{1}=0$. 
$I_{300}$

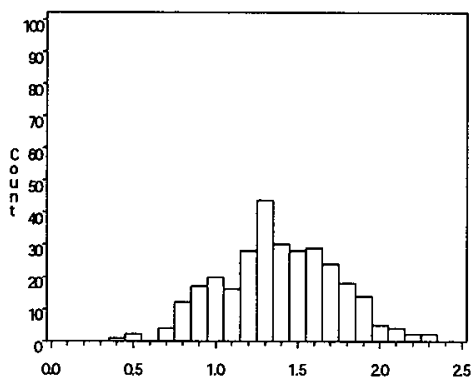

$a_{j}$

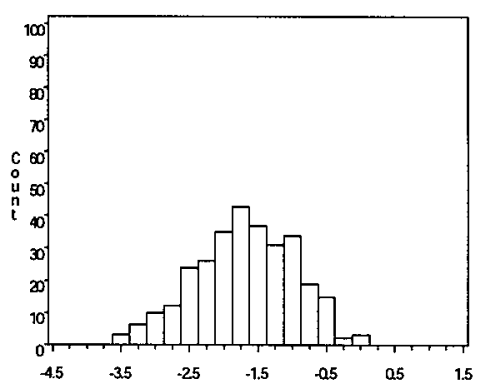

$b_{i 1}$

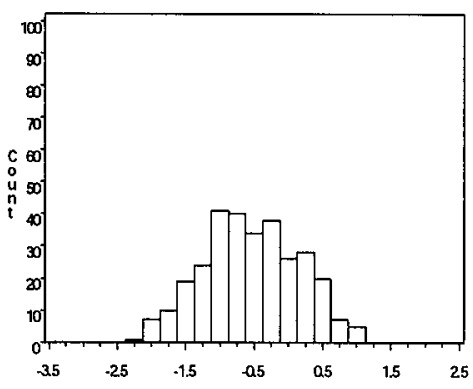

$b_{i 2}$
$I_{600}$

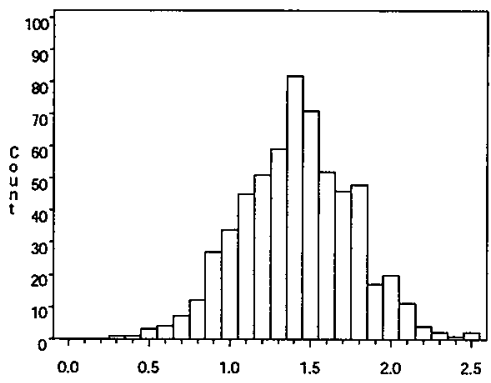

$a_{i}$

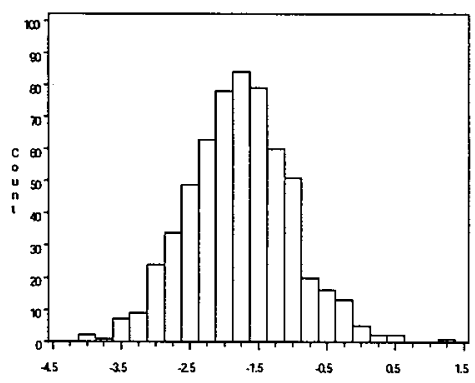

$b_{i 1}$

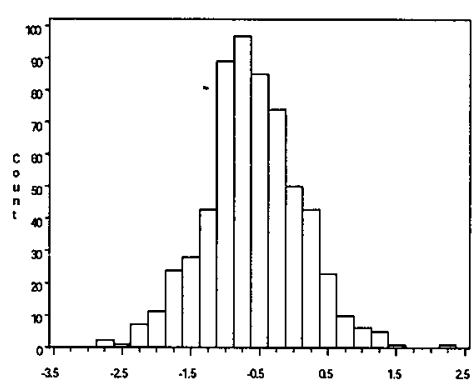

$b_{i 2}$

Figure 1. Histograms of item parameters distributions for pools $I_{300}$ and $I_{600}$ 


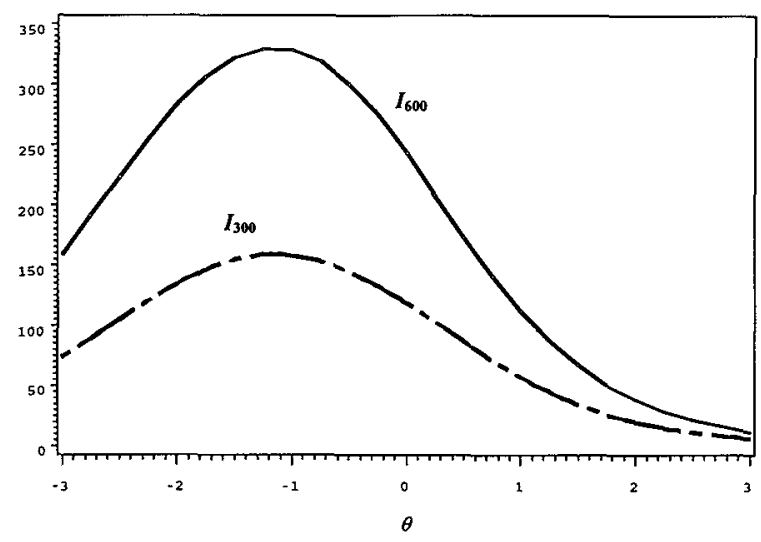

Figure 2. Item bank information functions

CAT implementation. The maximum likelihood estimator (MLE) of $\theta$ was computed using the Newton-Raphson method via the Fisher scoring equation for the GRM (see Baker, 1992, p.237-239 for more details). Given the estimate $\hat{\theta}_{n-1}, n=2,3, \ldots, 15$, based on $(n-1)$ administered items, the following $n$th item was selected in a way as to maximise $\Phi_{\mathrm{A}}\left(\operatorname{In} f_{i, \theta_{\mathrm{t}}}\right)$ in (5), $\Phi_{\mathrm{D}}\left(\operatorname{In} f_{i, \theta_{t}}\right)$ in (6), and $K_{i}\left(\theta_{0}\right)$ in (7), over the $99 \%$ confidence interval. The trait confidence interval of the $n$th step in the CAT implementation, $\Theta_{n}$, is defined as $\Theta_{n}=\left[\hat{\theta}_{n-1}-\delta_{n}\right.$, $\left.\hat{\theta}_{n-1}+\delta_{n}\right]$, with $\delta_{n}=\frac{c}{\sqrt{n}+1}$, where $\mathrm{c}$ is a constant selected according to a coverage probability (Chang and Ying, 1996). As used by Chen et al. (2000), herein $c=3$. Once the increment between the provisional estimate $\hat{\theta}_{n}$ and the preceding one $\hat{\theta}_{n-1}$ was too large in absolute terms, i.e. $\left|\hat{\theta}_{n}-\hat{\theta}_{n-1}\right| \geq 4$, the $n$th confidence interval together with $\hat{\theta}_{n}$ assumed the values used in the initialisation. The process stopped at $n=15$ selected items.

Evaluation measures. The ISCs performances were evaluated via the average bias (BIAS) and root mean squared error (RMSE), computed after 1000 replications:

$$
\operatorname{BIAS}(n, \theta)=\frac{1}{1000} \sum_{1000}\left(\hat{\theta}_{n}-\theta\right)
$$




$$
\operatorname{RMSE}(n, \theta)=\sqrt{\frac{1}{1000} \sum_{1000}\left(\hat{\theta}_{n}-\theta\right)^{2}} .
$$

The BIAS and RMSE were calculated for the A-, D- optimality criteria and the KL information criterion, for each true trait, $(\theta=-3,-2,-1,0,1,2,3)$, and eventually for each stage of the CAT test with $(n=1,2,3, \ldots, 15)$. In runs where the estimation of $\theta$ quickly degenerated, i.e. for trait values with monotone response patterns, the BIAS and RMSE were occasionally calculated with less than 1000 replications.

Item exposure rate. Exposure rate is a standard measure conveying how effective the items in a bank are being utilised. Clearly, this measure is dependent on the underlying trait distribution. It is defined as the relative frequency of an item $i$ selected throughout the test sessions (Cheng and Liou, 2003, Chang and Ying, 1999), and is computed as:

$$
\left.E R_{i}=\text { (frequency } \text { of } i \text { th item being selected }\right) / m \text {, }
$$

where $m$ represents the number of test sessions. In the current paper, the number of test sessions is replaced by the total number of replications for all trait values, i.e. maximum (1000 replications $\times 7$ traits).

\subsection{Results}

\subsubsection{Precision and accuracy}

A graphical representation of the RMSE and bias for estimation of trait levels, $(\theta=-3$, $-2,-1,0,1,2,3)$ can be seen from Figures 3 to 4 and Figures 5 to 6 , for pools $I_{300}$ and $I_{600}$, respectively. The interrupted, semi-interrupted and full lines correspond to the D- A- and KLcriteria, in this order. Regardless of the criteria, poor efficiency and accuracy can be observed for the higher traits $\theta=(2,3)$ especially for simulations wit pool $I_{300}$. This outcome confirms previous results from Lima Passos et al. (2004), which, among others, show that an ISC performance mirrors the information availability in the item bank. 


$$
\theta=-3 \quad \theta=-2 \quad \theta=-1
$$

RMSE

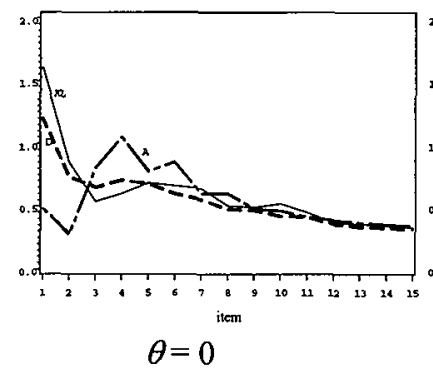

RMSE

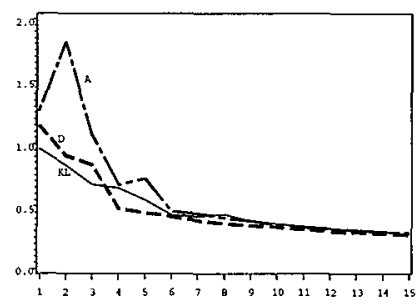

$\theta=3$

RMSE

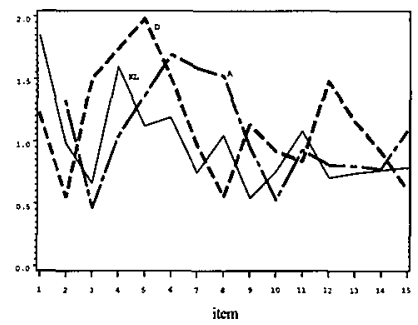

RMSE

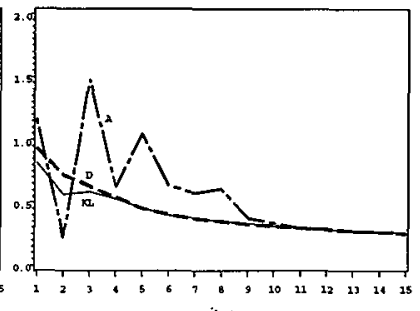

$\theta=1$

RMSE

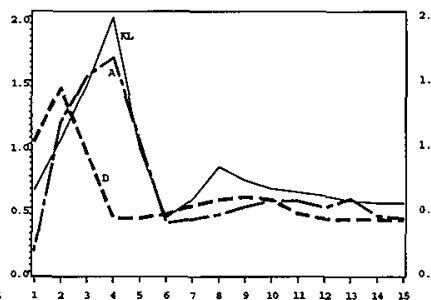

itan

RMSE

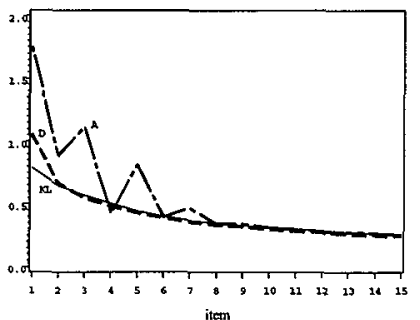

$\theta=2$

RMSE

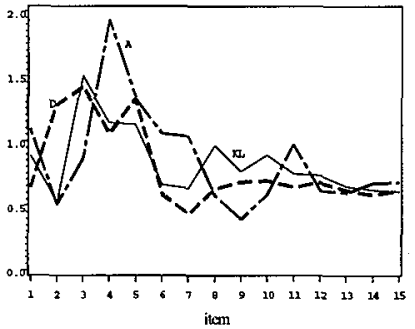

item

KL

D

$-1-\div-\div-\div$

Figure 3. RMSEs of trait estimates (item pool $I_{300}$ ).

In general, both pools produced comparable results at the end of the items administration. This is to say that between $I_{300}$ and $I_{600}$, the final outcome was similar, yet the course thereto not necessarily. The validity of this argument is especially evident for the Aoptimality criterion, which clearly yielded more instable RMSE/bias with pool $I_{300}$ than $I_{600}$. Conversely, the D- and KL-criteria little differed in their performances for both pools, manifesting greater robustness against instability under the two different item pool compositions. Thus estimation instability, expressed in form of RMSE/bias fluctuations, could be observed only for the higher traits, for which estimation did not converge easily because relative little information was available, and for the A-criterion with pool $I_{300}$. 

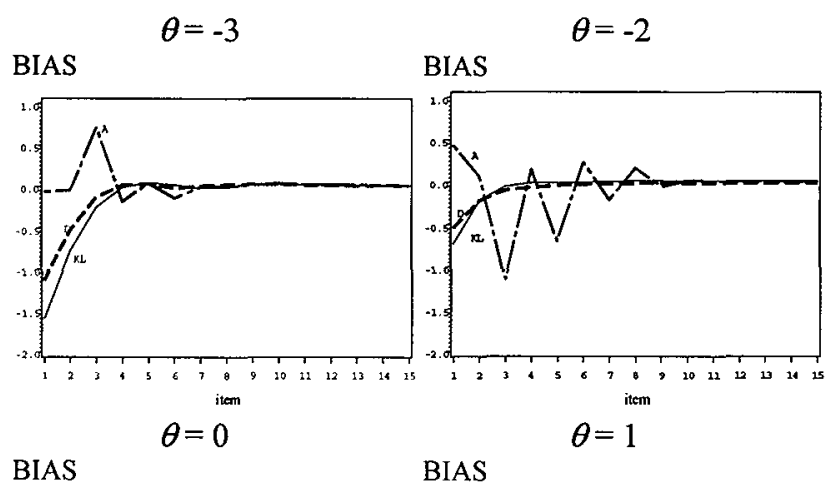

BIAS $\quad \theta=-1$

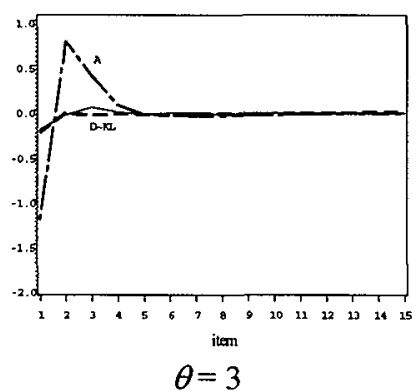

BIAS
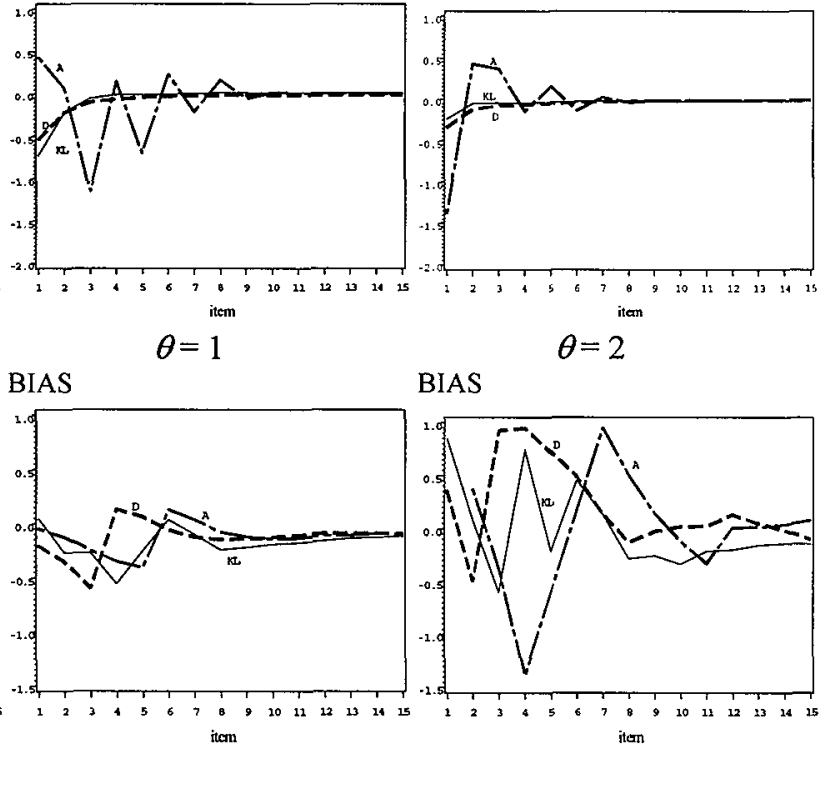

BIAS

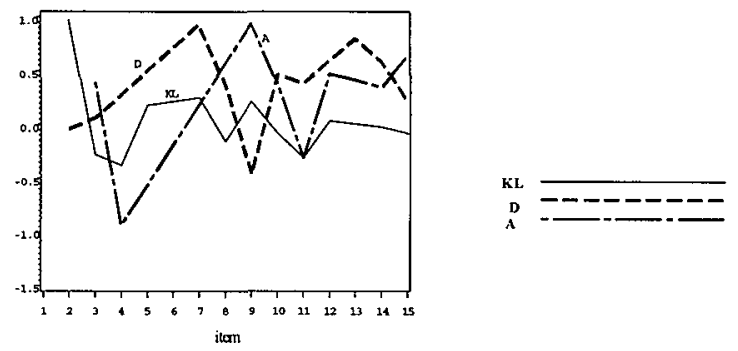

Figure 4. Bias of trait estimates (item pool $I_{300}$ ).

As previously mentioned, CAT early stage refers to the phase of estimation, in which estimates have not yet converged to the true trait value, after the administration of a few items. From the displayed pictures (Figures 3 to 6 ) it is evident that a clear-cut delimitation of this stage is difficult. To say exactly how many items the early stage comprises depends eventually on the speed of convergence to the true value, which in turn depends, on how much information a pool can offer for the underlying trait, on the assumed trait start-value, on the applied ISC, and of course on the sort of item (polytomous, graded items usually have more information on the latent trait than dichotomous items). 


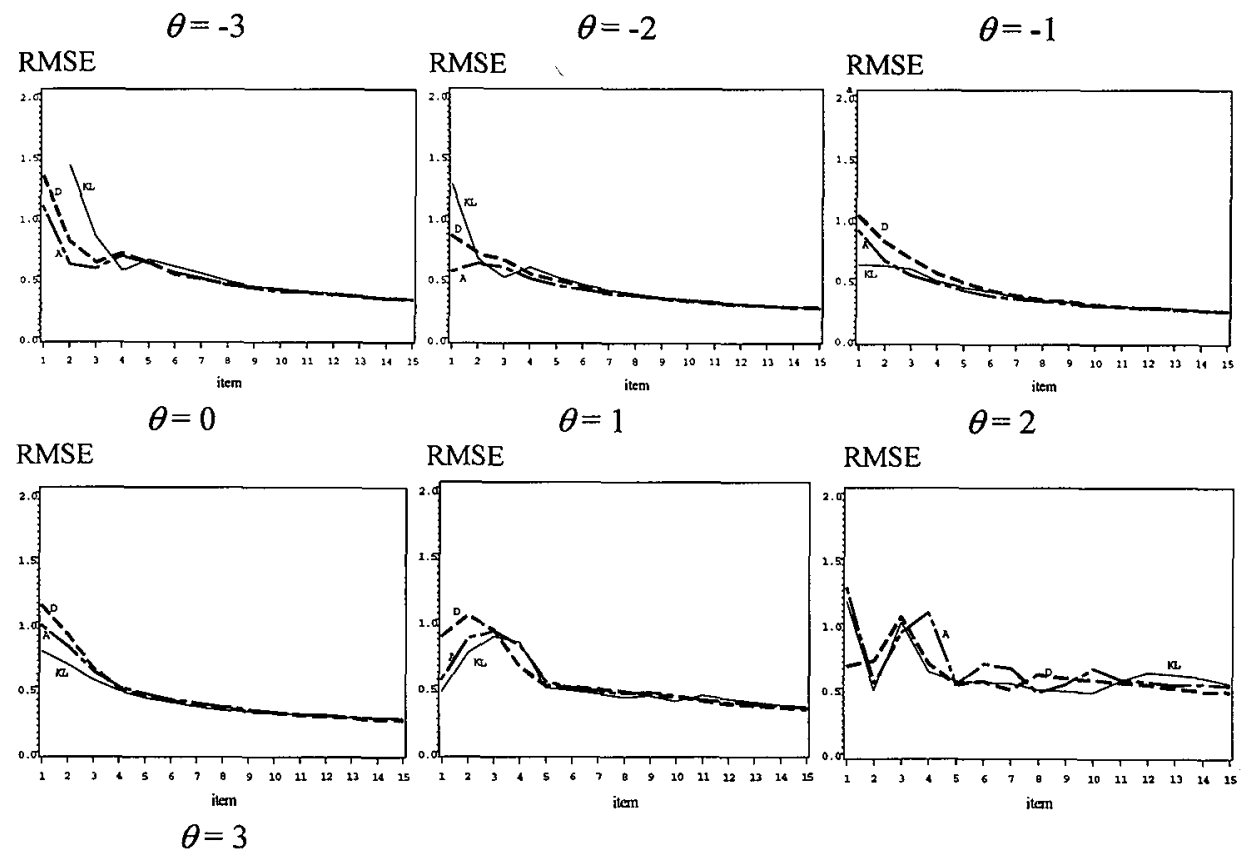

RMSE

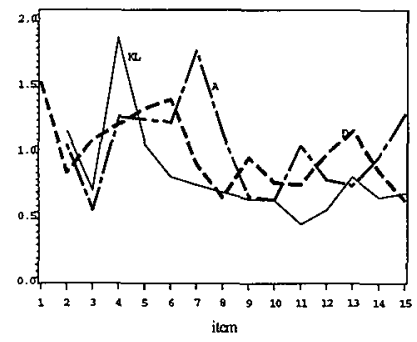

KL

D

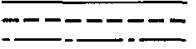

Figure 5. RMSEs of trait estimates (item pool $I_{600}$ ).

Nonetheless, two stages can be roughly distinguished, primarily for those traits for which convergence was attained. In the early stage, the RMSE/bias lines of the three criteria can be easily held apart from each other. In practice, this phase is characterised by little information about the trait value, what results in high RMSE/bias values. The second or interim stage, by contrast, shows a more stable, convergent and overlapping picture among the criteria. Here more information is available, since estimation is based on a longer string of responses. 
$\theta=-3$

BIAS

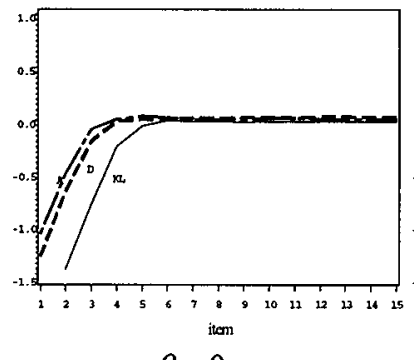

$\theta=0$

BIAS

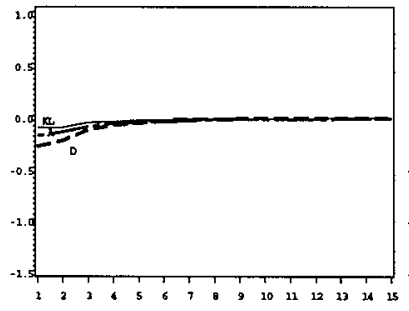

$\theta=3$

BIAS

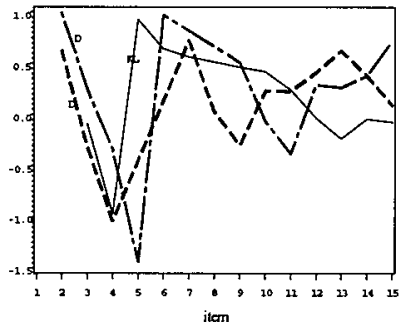

$\theta=-2$

BIAS

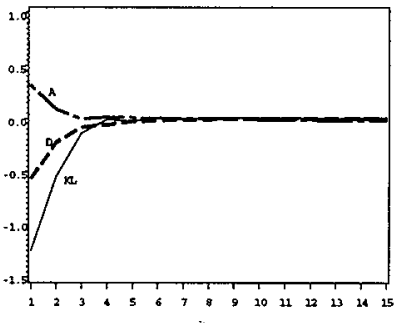

$\theta=1$

BIAS

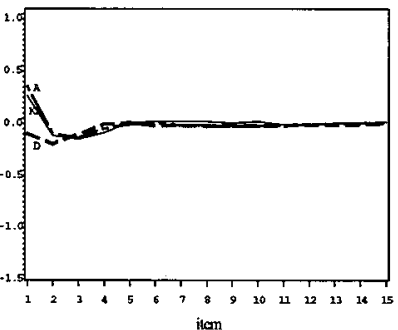

BIAS

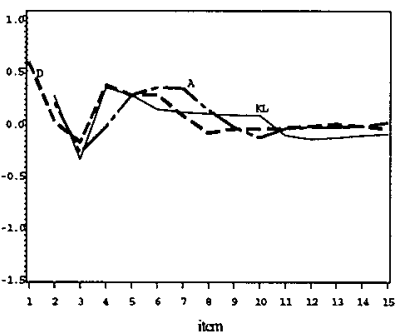

BIAS

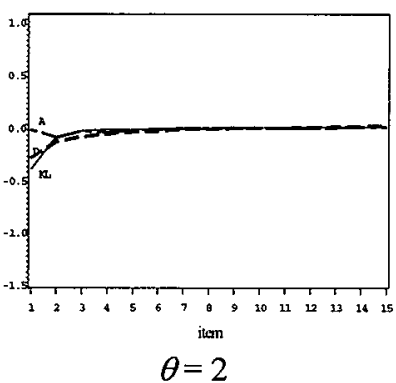

ki.

Figure 6. Bias of trait estimates (item pool $I_{600}$ ).

These results suggest the following: the three criteria manifest different ways of reacting to the lack of information about the trait value at the early stage. Their performances however, become similar as more information is gathered. The 'lack of information' is expressed in form of the width of the confidence interval $\Theta$, conveying the uncertainty of trait estimator: the larger the interval, the greater the uncertainty.

In the ensuing session, a closer investigation of the items targeted by the ISCs during these different stages is carried out with the objective to bare the nature of the RMSE/bias results. 


\subsubsection{Criteria selective strategies}

Changes undergone by the item parametric characteristics along the test for both pools can be followed in Figure 7 for the discrimination $\left(a_{i}\right)$ and location parameters $\left(b_{i 1}\right.$ and $\left.b_{i 2}\right)$. They allow for a visualisation of the items' parameters that are distinctly selected by the criteria as the test unfolds. For parsimonious reasons, only two of them, namely $\theta=-1$ and $\theta$ $=1$, are used to exemplify the criteria preferences. From these two traits, both item pools provide more information on the $\theta=-1$ than on $\theta=1$. Finally, to visualise the item pool effect, the curves related to $I_{300}$ and $I_{600}$ are plotted together, with the bold and normal lines associated with $I_{300}$ and $I_{600}$, respectively. The parameters' plots were obtained by averaging the selected item parameter values for each step over the total number of replications. T-tests for sample means of parameter values were carried out, mainly for the last step, $n=15$. Significant differences were verified above all in intra-criterion comparisons where the converging lines can be visibly de held apart, as, for instance, for the A-D and KL -criteria $a_{i}$ values between pools $I_{300}$ and $I_{600}$ for $\theta=-1$, and for $b_{i 1}, b_{i 2}$ values between pools $I_{300}$ and $I_{600}$, for $\theta=1$.

Item parameters. For the plots in Figure 7, in which the parameters' selection reaches a certain convergence, two stages can be discerned, analogously to the two estimation stages described for the RMSE and bias in the previous section. In the early stage, the choices of items by the $\mathrm{A}-\mathrm{D}$ - and $\mathrm{KL}$ - criteria are visibly different, whereas in the transition to the interim stage a convergence of parameters values is attained. The convergence of parametric values indicates overlap of item selection by the different criteria.

For the $a_{i}$ parameters (Figure 7), the D-criterion selected a relative low discriminative item at the outset for both pools, unlike the A- and KL-criteria, which started with more discriminative items. As more information is gathered, the parametric differences faded. This divergent/convergent pattern can be also found in the plots, where the sequential selection of location parameters is depicted (Figure 7). Note that the swifter convergence of $b_{i 1}$ and $b_{i 2}$ lines, the steadier the resulting RMSE/bias curves. 

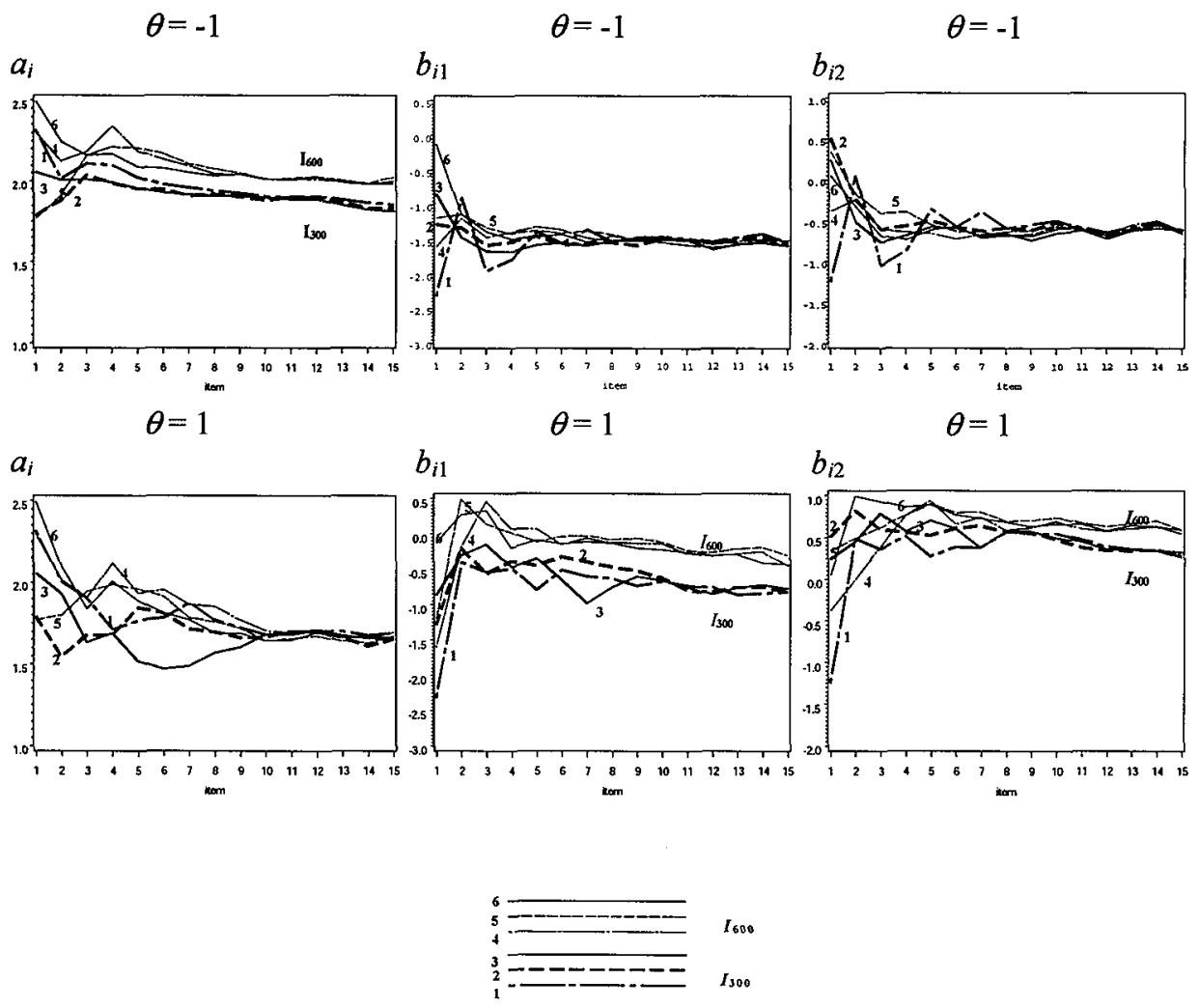

Figure 7. Item discrimination, $a_{i}$, and location parameters, $b_{i 1}$ and $b_{i 2}$

(1) A-criterion for $I_{300}$, (2) D-criterion for $I_{300}$ (3) KL-criterion for $I_{300}$

(4) A-criterion for $I_{600},(5) \mathrm{D}$-criterion for $I_{600}(6) \mathrm{KL}$-criterion for $I_{600}$

Item pools. In order to illustrate why the quality of trait estimation is associated with item parameters convergence, and this in turn is a function of the information availability in a pool, additional plots of item information functions are presented in Figure 8. These information functions were calculated based on the averaged item parameters values $a_{i,} b_{i 1}$ and $b_{i 2}$ of the last step, $n=15$ for each criterion, whose values are presented in Table 1. Depending on how much information is available for the underlying trait, the modes of the information functions of items selected at $n=15$ vary in their closeness to the true trait value (closer for $\theta=-1$ than $\theta=1)$. Note that the locations on the trait scale of these maximum information points coincide approximately with the value given by $\left(b_{i 1}+b_{i 2}\right) / 2$, i.e. the mode of the item's second response alternative on the trait scale $b_{i 2}$ (see section 2.1). 


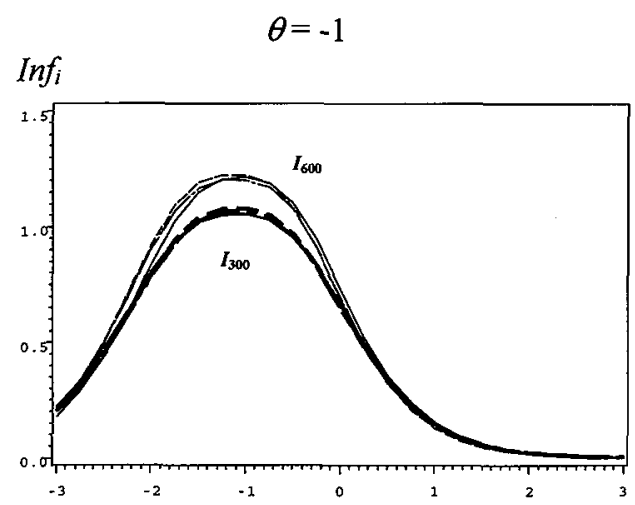

$\theta$

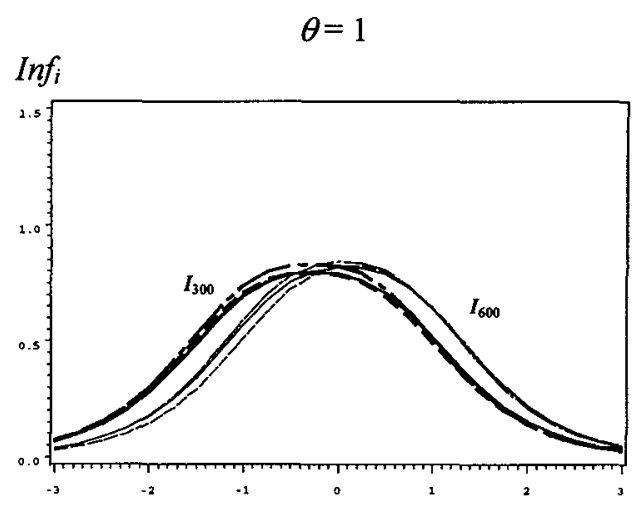

$\theta$

Figure 8. Information functions Inf of items selected by the A-D- and KL-criteria at step $n=15$ for estimation of traits $\theta=-1$ and $\theta=1$.

(1) A-criterion for $I_{300}$, (2) D-criterion for $I_{300}$ (3) KL-criterion for $I_{300}$

(4) A-criterion for $I_{600},(5)$ D-criterion for $I_{600}$ (6) KL-criterion for $I_{600}$

The differences in item pool composition explain the dissimilarities between the parametric plots of the two pools (Figure 7). Both $I_{300}$ and $I_{600}$ have the same mean and variance-covariance structure in terms of parametric distributions. However, due to its larger size, a greater number and diversity of parameter values has been generated for $I_{600}$. It is noteworthy that for the larger pool $I_{600}$ the $a_{i}$ lines (Figure 7) are for $\theta=-1$ visibly higher than for $I_{300}$ with $b_{j 1}$ and $b_{i 2}$ lines at the same level. As a result, the last items selected by the criteria for $\theta=-1$ have information functions (Figure 8) with a higher peak for $I_{600}$, but with similar location of modes as for $I_{300}$. This result shows that once estimation convergence is possible, items are selected, whose location parameters are well tuned the true trait, and the discrimination values are as high as the pool can offer. For the trait, for which relatively less informative/discriminative items were available $(\theta=1)$, the convergence of $a_{i}$ lines was delayed whereas a swifter convergence of the $b_{i 1}$ and $b_{i 2}$ lines could be observed (Figure 7). The higher ones, which are associated with the $I_{600}$, yield information functions, whose modes are slightly more shifted towards the true trait (Figure $8, \theta=1$ ). Figures 7 and 8 show that the 
smaller the difference given by $\left[\left(b_{i 1}+b_{i 2}\right) / 2-\theta\right]$, the better, i.e. the more accurate the tailoring between a selected item $i$ and the underlying trait $\theta$.

Table 1. Mean location and discrimination parameter values selected by the A- D- and KLcriteria for $n=15$.

\begin{tabular}{|c|c|c|c|c|c|}
\hline \multirow[b]{2}{*}{ ISC } & & \multicolumn{2}{|c|}{$\theta=-1$} & \multicolumn{2}{|c|}{$\theta=1$} \\
\hline & & $I_{300}$ & $I_{600}$ & $I_{300}$ & $I_{600}$ \\
\hline \multirow{3}{*}{ A } & $a_{i}$ & 1.913 & 2.041 & 1.692 & 1.694 \\
\hline & $b_{i 1}$ & -1.549 & -1.594 & -0.821 & -0.447 \\
\hline & $b_{i 2}$ & -0.636 & -0.646 & 0.277 & 0.551 \\
\hline \multirow{3}{*}{ D } & $a_{i}$ & 1.923 & 2.059 & 1.652 & 1.671 \\
\hline & $b_{i 1}$ & -1.560 & -1.601 & -0.810 & -0.300 \\
\hline & $b_{i 2}$ & -0.638 & -0.667 & 0.280 & 0.594 \\
\hline \multirow{3}{*}{ KL } & $a_{i}$ & 1.905 & 2.043 & 1.652 & 1.669 \\
\hline & $b_{i 1}$ & -1.590 & -1.510 & -0.771 & -0.429 \\
\hline & $b_{i 2}$ & -0.611 & -0.606 & 0.322 & 0.588 \\
\hline
\end{tabular}

To sum up, an analysis of all graphical displays in conjunction (Figures 1 to 7) suggests early stage instability to be above all associated with the speed at which a criterion succeeds in matching location parameters to the underlying trait. A criterion that allows for a quick convergence to the most suitable location parameters produces steady RMSE and bias curves.

\subsubsection{Criteria exposure rates}

For Figure 9, the items have been sorted according to their frequency of use. For a balanced item usage, i.e. a relative uniform frequency of selected items, these curves are expected to be relatively flat. 


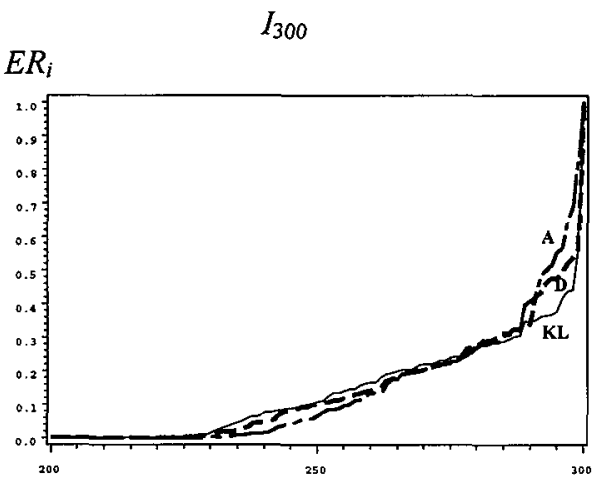

item

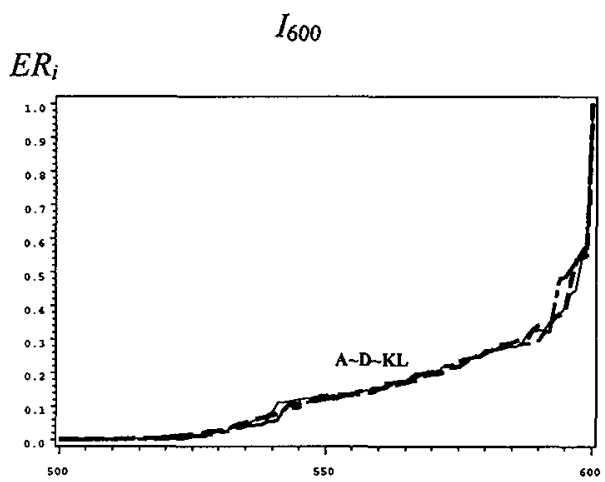

item

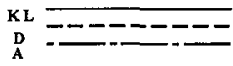

Figure 9. Item exposure rates, $E R_{i}$ of three ISCs for pools $I_{300}$ and $I_{600}$

From Figure 9 it is evident that all criteria produced high exposure rates for a few items, for both $I_{300}$ and $I_{600}$. For simulations with $I_{300}$, the D-and KL- exposure curves were marginally flatter than the A-curve, but little difference could be observed for simulations with $I_{600}$. Accordingly, the hypothesis put forward by Lima Passos et al. (2004) suggesting the D-criterion to be more flexible in its item selection could not be confirmed. Its full refutation, however, would be hasty. At least two reasons accounts for the obtained overexposure. Firstly, an effective use of the items in a pool was greatly impeded by a limited number of estimable trait values (only seven). Secondly, the first item selected by each of the criteria was the same, since it was based on the same initialisation trait interval $\Theta_{1}=[-3,3]$, irrespective of the underlying trait. These first items differed among the criteria, though. It is assumed that the exposure rates can be possibly improved by changing the test premises with respect to a random start-item and to a more diversified trait distribution. It remains to be seen, whether these changes would affect all criteria equally. 


\subsection{Concluding remarks}

There is a host of aspects to be considered in the choice of suitable ISCs. Efficiency and accuracy of measurement are of major relevance. Equally important is the way a criterion tackles uncertainty during the critical CAT early stage, the phase of estimation where little is known about the trait's exact location on the latent scale. In the face of uncertainty, all three criteria differed in their selective strategies.

The choice of a relative low discrimination parameter items at the beginning turned out to be a robust alternative, as shown by the D-criterion. These items usually offer a small but broad information coverage of the trait range, getting around the attenuation paradox, leading a gradual decrease of the trait's confidence interval, producing thereby relative stable estimates. The KL-criterion, in turn, achieved a similar end, in spite of having selected discriminative items at the start. The KL-outcome is likely explained by its global information approach, lending supporting evidence to Chang and Ying (1996) hypothesis that the global information is more robust against early stage instability than a local information criterion. However, this statement has been shown to be valid for the GRM and should not be indiscriminately extended to other dichotomous or polytomous IRT models. The findings of Lima Passos et al. (2004) with the Nominal Response Model, for instance, endorsed, only partially, KL's greater robustness. As for the A-criterion, it required more time to converge with $I_{300}$, but not with $I_{600}$. This suggests that the start-choice of a discriminative item by a local information (FI) criterion does not obligatory imply early stage instability, but possibly increase the propensity to it.

The obtained outcome on item exposure was not conclusive enough to allow for a solid comparison among the criteria exposure rates. This preliminary finding proves once again that one should not expect from an optimal selection criteria to make a uniform use of items in a pool without a proper exposure controlling measure. However, the results did hint at a greater flexibility of the D-and $\mathrm{KL}$ - criteria for the smaller pool, motivating further investigations. It has been seen that the higher the parametric diversity in the pool, the more similar and overlapping the item selection among the criteria. Consequently, their exposure rates became more concordant.

Finally, on the evidences of early stage stability, a safe recommendation for adaptive testing with the GRM would have to give precedence to the $\mathrm{D}$ - and $\mathrm{KL}$ - over the A-criterion. Yet, simulations with the D- and A-criteria took, in general, 1/3 of the running time needed for KL-simulations. The additional aspect of less CPU time gives the D-optimality criterion a numerical advantage. Without compromising precision and accuracy, the D-criterion's simple 
numerical handling and relative stability of estimation substantiate its feasibility and suitability for a CAT environment. 
Chapter 5

116 


\section{On polytomous versus dichotomous} items: The quest of information revisited 


\title{
On polytomous versus dichotomous items: The quest of information revisited
}

\begin{abstract}
In IRT literature there is a common view that places polytomous above dichotomous items in their role of extracting information about an examinee's latent trait level from his/her response pattern. This view, however, should not be taken for granted. The validity of such standpoint is model-dependent. While this is unambiguously true for models with graded response pattern, this might not necessarily apply to nominal response data. In this paper, a dichotomous-polytomous transition and the ensuing changes on Fisher's information will be evaluated for the nominal response model. A comparison will show that for nominal data the upper hand of polytomous items in terms of Fisher's information is not always justified. An optimal test design based on different item selection criteria is carried out in order to exemplify when the use of binary items might be preferable to multi-categorical items.
\end{abstract}

Keywords: Fisher's information, 2PL, NRM, item's efficiency 


\section{On polytomous versus dichotomous items: The quest of information revisited}

\subsection{Introduction}

Item Response Theory (IRT) models have been increasingly employed in tests measuring educational and psychological traits. The advantages offered by IRT in comparison to classical test theory are well known and reasonably established for dichotomous IRT models (Hambleton and Swaminathan, 1985; Hambleton, Swaminathan and Rogers, 1991; Hambleton and Jones, 1993). These advantages, which are primarily associated with the concepts of person invariance of item parameters, item invariance of person parameter and information function, have been shown to apply also to polytomous IRT models (Mellenbergh, 1995).

In the Handbook of Modern Item Response Theory (Van der Linden \& Hambleton, 1997 , p.30-31), the authors state that the intent of polytomous items is to extract more information about an examinee's ability level from a test item than simply whether or not the examinee answers the item correctly. This tendency of regarding polytomous items as generally more informative than dichotomous is not at all uncommon. In general, polytomous items and their descriptive models are taken to be better suited to the task of extracting information from an examinee's response pattern, mainly due to its differential treatment of incorrect answers. By contrast, dichotomous items are not able to distinguish between an incorrect answer, on the one hand, and a differentiated distribution of scores over incorrect alternatives, on the other hand. Binary items and their models disregard the 'distinct identity of incorrect alternatives' (Thissen and Steinberg, 1984).

There are several studies that indicate that picking one of the wrong alternatives is a manifestation of a deliberate preference, which depends on the latent trait level, $\theta$ (Nedelsky, 1954, Levine and Drasgow, 1983, Tatsuoka, 1983). The many-sided perspective into the wrong response pattern offered by polytomous items turned out to be useful in the quest of optimisation of Qestimation. Studies carried out by Bock (1972), Levine and Drasgow (1983), Thissen (1976), Thissen and Steinberg (1984) and De Ayala (1992) have shown that precision of measurement can be obtained by considering information of incorrect alternatives. However, as Thissen and Steinberg (1984) and Thissen, Steinberg and Fitzpatrick (1989) point out, there are a few complexities involved in the use of multiplechoice items for $\theta$-estimation. 
Before the introduction of polytomous models, items with multiple response categories used to be analysed by shrinking the multiple options into correct/incorrect alternatives. Their evaluation would follow by applying dichotomous models. Loss of information on the latent trait was the immediate result of such 'dichotomisation'. The advent of polytomous models brought an end to the discrepancy between data and model, allowing for each response option to be described by its own characteristic function. As a result, information on $\theta$, provided by each individual item category, even if wrong, could be retrieved for the purpose of trait estimation.

If brought in association with the dichotomisation of multi-categorical items, the prevailing standpoint favouring polytomous items in terms of information on $\theta$ is justified. As De Ayala (1992) states: 'It is obvious that the dichotomisation of an examinee's response ignores any partial knowledge that the examinee may have of the correct answer and, as a result, this information cannot be used for $\theta$ estimation'. Dodd, De Ayala and Koch (1995) support this statement and extend it to all polytomous models discussed in their paper, which represent a wider range of graded and nominal response models. However, the information loss via dichotomisation is not sufficient to validate polytomous items' upper hand in the information contest. Which of the two types of items has more information depends on the assumed model.

Berger and Mathijssen (1997), for example, have shown that for the Nominal Response Model (NRM) dichotomous items can surpass polytomous items in terms of information on $\theta$. This finding is at odds with the established view associating polytomous items with more information. It suggests that category information can, but might not necessarily pay-off in the final precision of a maximum likelihood estimator $\hat{\theta}$.

The objective of the present study is to revisit the dichotomous versus polytomous information debate, by challenging, at least partly, the common view that ascribes to multicategorical items a greater efficiency in obtaining information on $\theta$. For this purpose an investigation of Fisher's information is carried out on the basis of the two parameters logistic model (2PL) and the three categories Nominal Response Model (NRM). In section 2 and 3 the models will be described together with their corresponding Fisher's information. An evaluation of the latter will take place in section 4 . Subsequently, in section 5 , a more realistic perspective of the polytomous/dochotomous debate is offered via an optimal test design. This is carried out based on item pools composed of both polytomous and dichotomous items. The obtained results are discussed and explanations provided in section 6 , which is followed by 
some final remarks upon which test circumstances the choice of polytomous over dichotomous, or vice-versa, is justified in section 7 .

\subsection{The models}

Thissen and Steinberg (1986) developed a taxonomic system for IRT models. According to it, there are basically two generic subdivisions for polytomous models, reflecting two conceptually distinct approaches for estimating the $k_{i}$ alternative response functions of item $i$. The classification makes a distinction between the difference and the divided-by-total models. The latter require simultaneous estimation of all $k_{i}$ categorical probability functions, whereas the former replaces simultaneous by subsequent and individual estimation of $k_{i}-1$ cumulative probability functions in a binary fashion (Abrahamowicz and Ramsay, 1992).

There are several multi-categorical divided-by-total models presented in the Thissen and Steinberg classification. All the models can be, however, regarded as generalisations of the most basic form, the Nominal Response Model (NRM), firstly introduced by Bock (1972). As the name itself says, the NRM is one of few polytomous models that are suitable for items with nominal response format, since they do not require any ordering of the response categories. The equation that describes the NRM is:

$$
P_{i g \mid \theta}=\frac{\exp \left(c_{i g}+a_{i g} \theta\right)}{\sum_{g=1}^{k_{i}} \exp \left(c_{i g}+a_{i g} \theta\right)},
$$

where $a_{i g}$ is the discrimination parameter of category $g$ of item $i, i=1, \ldots, n, c_{i g}$ is the location parameter associated with category $g$ of item $i, k_{i}$ is the number of categories of item $i$ with $g$ $=1,2, \ldots, k_{i}, \theta$ is the latent trait variable or ability level of the test-taker, and $P_{i g \mid \theta}$ is the probability of examinee with ability $\theta$ of choosing category $g$ of item $i$, i.e. the probability response function.

The maximum likelihood estimation of NRM item parameters requires identification constraints on the values of discrimination and location parameters $a_{i g}$ and $c_{i g}$, respectively. There are two commonly used alternative constraints: In this paper a constraint will be used, in which the categories are ordered according to their discrimination values, i.e. 
$a_{i 1}<a_{i 2} \ldots<a_{i g}$, and the value of both discrimination and difficulty of the first category is taken to be zero, i.e. the $a_{i 1}=c_{i 1}=0$ (see also Heinen, 1996). Another possibility has been introduced by Bock (1972), see also Baker (1992, p. 253) and forces $\sum_{g} a_{i g}=0$ and $\sum_{g} c_{i g}=0$

The dichotomous $2 \mathrm{PL}$ can be seen as a special case of the NRM with only two categories. In this case, the equation described in (1) becomes

$$
P_{i \theta}=\frac{\exp \left(c_{i}+a_{i} \theta\right)}{1+\exp \left(c_{i}+a_{i} \theta\right)}
$$

where $P_{i \mid \theta}$ represents the probability of selecting the correct response of a binary item described by a $2 \mathrm{PL}$ model. The description of variables remains the same as above with the difference that the categories index $g$ of item parameters has been dropped.

\subsection{Fisher's Information}

The objective of any item, regardless of its polytomous or dichotomous nature, is to estimate the latent trait variable $\theta$. In the theory of statistical estimation, Fisher's Information is the amount of information contained in the data about an unknown parameter $\theta$ and is defined as minus the expected value of the second derivative of the logarithm of the likelihood function, with respect to the parameter $\theta$.

$$
\operatorname{Inf}=-E\left(\frac{\partial^{2} \log L}{\partial \theta^{2}}\right)
$$

The likelihood function $L$, in turn, is model dependent. Any change in the model has a direct consequence of altering the likelihood function, and hence the information. For the NRM model Fisher's Information of an item $i$ on a single trait is: 


$$
\operatorname{Inf} f_{i}=\left\{\sum_{g} a_{i g}^{2} P_{i g \mid \theta}-\left[\sum_{g} a_{i g} P_{i g \mid \theta}\right]^{2}\right\},
$$

whereas for the 2PL, Fisher's Information of an item $i$ on $\theta$ is given by:

$$
\operatorname{Inf} f_{i}=a_{i}^{2} P_{i \theta}\left(1-P_{i \theta}\right)
$$

The index $g$ in (5) runs through the number of categories $k_{i}$ of item $i$, i.e. $g=1,2, \ldots$, $k_{i}$ with $a_{i g}$ standing for their corresponding discrimination parameters. In case $k_{i}=2$, i.e. an item with two categories (bearing in mind that $a_{i 1}=0$ ), the item information, as defined in (5), becomes the same expression as given by (6).

It should be noted that the total item information function is the sum over all categories information. This means the category information, Infig, represents a portion of the item information (5) that can be obtained by multiplying the latter by the category's conditional probabilities, i.e. $\operatorname{Inf} f_{i g}=\operatorname{Inf} f_{i} \times P_{i g \mid \theta}$.

\subsection{Exploration of conditional probabilities}

\subsubsection{Method}

For the NRM, Fisher's Information is defined in (5). By exploring all possible probabilities $P_{i g \mid \theta}$ permutations (shortly, $p$-combinations) of choosing the response categories, under the constraint that $\sum_{g} P_{i g \mid 0}=1$, it can be shown which one yields the highest information value. Berger and Mathijssen (1997) have proven that the maximum value of Fisher information is obtained when these NRM items behave 'dichotomously', i.e. when the probability for their intermediate categories to be selected converges to zero. Note that for calculating $\operatorname{Inf} f_{i}$, as in the equations (5) the item's location parameter is not of primary interest, only the conditional probabilities and the item's discrimination parameters.

To evaluate the effect of diverse $a_{i g}$ values on Fisher's information, three different sets of discrimination parameters, $a_{i g}$, have been used, i.e. set 1 with $a_{i g}=[0,0.5,2]$, set 2 with $a_{i g}$ $=[0,1,2]$ and set 3 with $a_{i g}=[0,1.5,2]$, such that $a_{i 1}<a_{i 2}<a_{i 3}$. When the discrimination parameters of a three categories item are arrayed in such an order, the first category is represented by the monotonically decreasing curve and the last by the monotonically 
increasing curve in an ICCC plot. The intermediary category has a unimodal curve (see Figure 1). Let it be reminded that the ordering $a_{i g}$ is just an artefact, which is needed in connection to $a_{i 1}=0$ to facilitate the estimation procedure in practice.

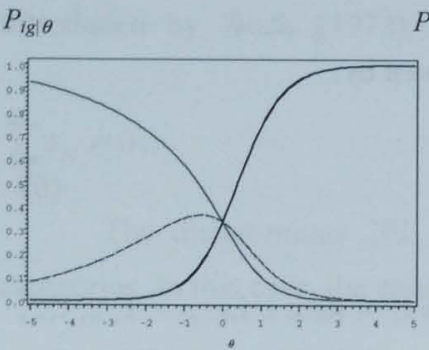

(a) Item $1, a_{12}=0.5$
$P_{i g \mid \theta}$

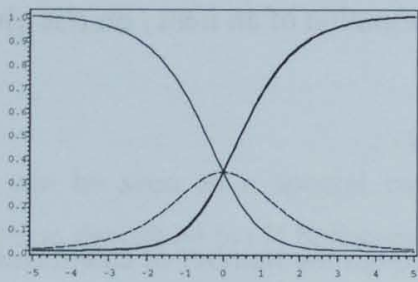

(b) Item $2, a_{22}=1.0$
$P_{i g \mid \theta}$

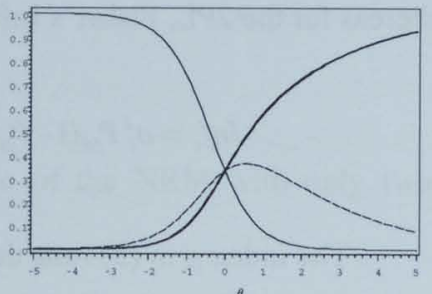

(c) Item $3, a_{32}=1.5$

Figure 1. Item Categories Characteristic Functions (ICCCs) of 3 categories items described by NRM. For all three items $a_{i 1}=0, a_{i 3}=2$ and $c_{i g}=0$.

\subsubsection{Results}

The information $I n f_{i}$ in (5) is computed for all possible permutations of $P_{i g} \theta$, which varies between 0 and 1 with $\sum_{g} P_{i g \theta}=1$. The attained information values, plotted against the p-combinations, are graphically displayed in Figure 2(a), 2(b) and 2(c) for the three different sets of discrimination parameters.

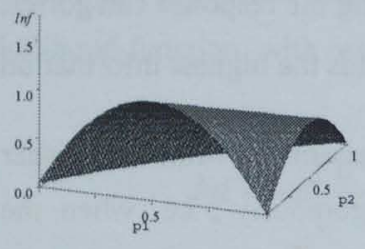

(a) $a_{i 1}=0$ $a_{i 2}=0.5$ $a_{i 3}=2$

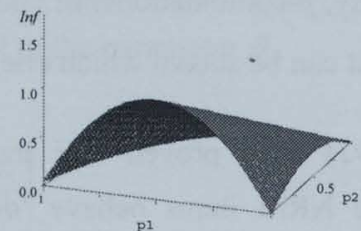

(b) $a_{i 1}=0$ $a_{i 2}=1$ $a_{i 3}=2$

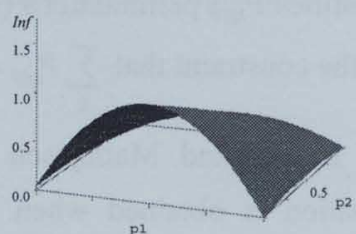

(c) $a_{i 1}=0$ $a_{i 2}=1.5$ $a_{i 3}=2$

Figure 2. Plot of Inf against conditional probabilities $P_{i g \mid \theta}$ for $g=1$ and 2 for the 3 categories NRM. The conditional probability for the third category is implicit since $\sum_{g} P_{i g \mid \theta}=1$. 
In Figure 2, all graphics have their maximum values $\operatorname{In} f_{\max }=1$ at the $p$-combinations $P_{i|| \theta}=0.5$ and $P_{i 2 \mid \theta}=0$, thus, $P_{i 3 \mid \theta}=0.5$, as proven by Berger and Mathijssen (1997) - see also the results of Table 1 . The difference among these graphics relates to the flattening of the surfacecurve along the $P_{i 2 \mid \theta}$ axis, as the value of $a_{i 2}$ approaches the value of $a_{i 3}$.

In Table 1 the information values are arranged in a crescent order from zero up to the maximum value for all three $a_{i g}$ sets ( $\max \operatorname{Inf} f_{\max }=1$, underlined and in bold). By using the increment of 0.1 for $P_{i g \mid \theta} 66$ values of $p$-combinations were obtained. The Inf values are related to $P_{i g \mid \theta}$ instead of $\theta$. Nevertheless, $P_{i g \mid \theta}$ indicate a relative position of $\theta$. The ordering of the $p$-combinations as given in Table 1 reflects implicitly a relative ordering of the underlying trait values. For example, the $p$-combination $\left[P_{i 1 \mid \theta}=0.8\right.$ and $P_{i 2 \mid \theta}=0$, thus, $\left.P_{i 3 \mid \theta}=0.2\right]$ is associated with a relatively lower trait value than $\left[P_{i 1 \mid \theta}=0.2\right.$ and $P_{i 2 \mid \theta}=0$, thus, $\left.P_{i 3 \mid \theta}=0.8\right]$. This interpretation is possible due to the constraint, which sets $a_{i 1}<a_{i 2}<a_{i 3}$. The probability of selecting the category with the monotonically increasing curve is thus $P_{i 3 \mid \theta}$.

There are two perspectives from which Table 1 can be scrutinised. Firstly, the maximum information is given by the $p$-combination $P_{i|| \theta}=0.5, P_{i 2 \mid \theta}=0$ and $P_{i 3 \mid \theta}=0.5$. This combination is identical to a dichotomously scored item, described by the 2PL, whose discrimination parameter equals the difference between the maximum and minimum values of discrimination parameters in a NRM item, i.e. $\left[a_{i g(\max )}-a_{i g(\min )}\right]=a_{j}$. This is true regardless of the identification constraint used. This can be seen by equalising equations (5) and (6) with $P_{i 2 \mid \theta}=0$. Herein $a_{i 1}<a_{i 2}<a_{i 3}, a_{i 1}=0$, making $a_{i}=a_{i 3}=2$. In fact, by applying the same numerical exploration to a $2 \mathrm{PL}$ information function, as defined in equation (6), with $a_{i}=2$, the obtained information values and their corresponding dichotomous $p$-combinations are equal to the ones highlighted in bold in Table 1 , with $P_{i 2 \mid \theta}=0$. Although a $p$-combination with either $P_{i|| \theta}=0$ or $P_{i|| \theta}=0$ is also dichotomous, there is a reason for not highlighting them. To obtain a solid comparative basis, $\left[a_{i g(\max )}-a_{i g(\min )}\right]$ needs to be set equal $a_{i}$, thereby levelling the effect of the discrimination parameters have on Fisher's information. For dichotomous $p$ combinations with either $P_{i 1 \mid \theta}=0$ or $P_{i 3 \mid \theta}=0$, the corresponding 2PL item would no longer have its $a_{i}=2$, but rather a smaller value, i.e. $\left[a_{i g(\max )}-a_{i g(\min )}\right]>a_{i}$. This inequality would automatically make the binary item less informative. This can be seen, for instance, in Figure 2, where the Inf values are reduced along the $P_{i 2 \mid \theta}$ axis, as the value of $a_{i 2}$ converges to the value of $a_{i 3}$ for the dichotomous $p$-combinations where $P_{i l \mid \theta}=0$.

The second result from Table 1 relates to the displayed arrangement of information values and their corresponding conditional probabilities. This display gives some indication of how the information provided by dichotomous $p$-combinations stand in relation to the 
polytomous ones. Irrespective of which values $a_{i 2}$ takes ( $a_{i 1}$ and $a_{i 3}$ are fixed) the maximum information remains the one yielded by the $P_{i 1 \mid \theta}=0.5, P_{i 2 \mid \theta}=0, P_{i 3 \mid \theta}=0.5$ dichotomous $p$ combination. Yet, for sets 1 and 3 , there is a greater number polytomous $p$-combinations in between the dichotomous ones, highlighted in bold. This effect of $a_{i g}$-assemblage on the shape and maximum value of the items' information functions can be further visualised by means of an example with three categories items, all of which with the same $a_{i g(\max )}, a_{i g(\min )}$ and $c_{i g}$ (Figure 3). As in Table 1, they differ only in the discrimination parameter of the second category $a_{i 2}$. Their information functions together with the information function of the binary item, whose discrimination parameter $a_{i}=\left[a_{i g(\max )}-a_{i g(\min )}\right]$ and location parameter $c_{i}=c_{i g}=0$ are displayed. It can be seen in Figure 3 that as the $a_{i g}$-variance increases, the information curves become narrower but higher.

Table 1. All $p$-combinations for the NRM with 3 sets of discrimination parameters, for which $a_{i 1}=0$ and $a_{i 3}=2$

Set 1: $a_{i 2}=0.5$

\begin{tabular}{llll}
\hline Inf & $P_{i|| \theta}$ & $P_{i 2 \mid \theta}$ & $P_{i 3 \mid \theta}$ \\
& & & \\
\hline 0.0000 & 0.0 & 0.0 & 1.0 \\
0.0000 & 0.0 & 1.0 & 0.0 \\
0.0000 & 1.0 & 0.0 & 0.0 \\
0.0225 & 0.0 & 0.1 & 0.9 \\
0.0225 & 0.0 & 0.9 & 0.1 \\
0.0400 & 0.0 & 0.2 & 0.8 \\
0.0400 & 0.0 & 0.8 & 0.2 \\
0.0525 & 0.0 & 0.3 & 0.7 \\
0.0525 & 0.0 & 0.7 & 0.3 \\
0.0600 & 0.0 & 0.4 & 0.6 \\
0.0600 & 0.0 & 0.6 & 0.4 \\
0.0625 & 0.0 & 0.5 & 0.5 \\
0.2025 & 0.1 & 0.9 & 0.0 \\
0.2025 & 0.9 & 0.1 & 0.0 \\
0.2400 & 0.1 & 0.8 & 0.1 \\
0.2725 & 0.1 & 0.7 & 0.2 \\
0.3000 & 0.1 & 0.6 & 0.3 \\
0.3225 & 0.1 & 0.5 & 0.4 \\
0.3400 & 0.1 & 0.4 & 0.5 \\
0.3525 & 0.1 & 0.3 & 0.6 \\
$\mathbf{0 . 3 6 0 0}$ & 0.1 & 0.0 & 0.9 \\
0.3600 & 0.1 & 0.2 & 0.7 \\
0.3600 & 0.2 & 0.8 & 0.0 \\
0.3600 & 0.8 & 0.2 & 0.0 \\
0.3600 & 0.9 & 0.0 & 0.1 \\
0.4125 & 0.2 & 0.7 & 0.1 \\
0.4600 & 0.2 & 0.6 & 0.2 \\
0.4725 & 0.3 & 0.7 & 0.0 \\
0.4725 & 0.7 & 0.3 & 0.0 \\
0.5025 & 0.2 & 0.5 & 0.3 \\
0.5025 & 0.8 & 0.1 & 0.1 \\
0.5400 & 0.2 & 0.4 & 0.4 \\
0.5400 & 0.3 & 0.6 & 0.1 \\
0.5400 & 0.4 & 0.6 & 0.0 \\
0.5400 & 0.6 & 0.4 & 0.0 \\
0.5625 & 0.5 & 0.5 & 0.0 \\
& & &
\end{tabular}

Set $2: a_{i 2}=1$

$\overline{\text { Inf }} P_{i|| \theta} \quad P_{i 2 \mid \theta} \quad P_{i 3 \mid \theta}$

\begin{tabular}{llll}
\hline 0.00 & 0.0 & 0.0 & 1.0 \\
0.00 & 0.0 & 1.0 & 0.0 \\
0.00 & 1.0 & 0.0 & 0.0 \\
0.09 & 0.0 & 0.1 & 0.9 \\
0.09 & 0.0 & 0.9 & 0.1 \\
0.09 & 0.1 & 0.9 & 0.0 \\
0.09 & 0.9 & 0.1 & 0.0 \\
0.16 & 0.0 & 0.2 & 0.8 \\
0.16 & 0.0 & 0.8 & 0.2 \\
0.16 & 0.2 & 0.8 & 0.0 \\
0.16 & 0.8 & 0.2 & 0.0 \\
0.20 & 0.1 & 0.8 & 0.1 \\
0.21 & 0.0 & 0.3 & 0.7 \\
0.21 & 0.0 & 0.7 & 0.3 \\
0.21 & 0.3 & 0.7 & 0.0 \\
0.21 & 0.7 & 0.3 & 0.0 \\
0.24 & 0.0 & 0.4 & 0.6 \\
0.24 & 0.0 & 0.6 & 0.4 \\
0.24 & 0.4 & 0.6 & 0.0 \\
0.24 & 0.6 & 0.4 & 0.0 \\
0.25 & 0.0 & 0.5 & 0.5 \\
0.25 & 0.5 & 0.5 & 0.0 \\
0.29 & 0.1 & 0.7 & 0.2 \\
0.29 & 0.2 & 0.7 & 0.1 \\
0.36 & 0.3 & 0.6 & 0.1 \\
0.36 & 0.1 & 0.6 & 0.3 \\
0.36 & 0.9 & 0.0 & 0.1 \\
0.40 & 0.2 & 0.6 & 0.2 \\
0.41 & 0.4 & 0.5 & 0.1 \\
0.41 & 0.1 & 0.1 & 0.8 \\
0.41 & 0.1 & 0.5 & 0.4 \\
0.41 & 0.8 & 0.1 & 0.1 \\
0.44 & 0.5 & 0.4 & 0.1 \\
0.44 & 0.1 & 0.4 & 0.5 \\
0.44 & 0.1 & 0.2 & 0.7 \\
0.44 & 0.7 & 0.2 & 0.1
\end{tabular}

Set $3: a_{i 2}=1.5$

\begin{tabular}{clll}
\hline Inf & $P_{i I \theta}$ & $P_{i 2 i \theta}$ & $P_{i 3 \theta}$ \\
& & & \\
\hline 0.0000 & 0.0 & 0.0 & 1.0 \\
0.0000 & 0.0 & 1.0 & 0.0 \\
0.0000 & 1.0 & 0.0 & 0.0 \\
0.0225 & 0.1 & 0.9 & 0.0 \\
0.0225 & 0.9 & 0.1 & 0.0 \\
0.0400 & 0.2 & 0.8 & 0.0 \\
0.0400 & 0.8 & 0.2 & 0.0 \\
0.0525 & 0.3 & 0.7 & 0.0 \\
0.0525 & 0.7 & 0.3 & 0.0 \\
0.0600 & 0.4 & 0.6 & 0.0 \\
0.0600 & 0.6 & 0.4 & 0.0 \\
0.0625 & 0.5 & 0.5 & 0.0 \\
0.2025 & 0.0 & 0.1 & 0.9 \\
0.2025 & 0.0 & 0.9 & 0.1 \\
0.2400 & 0.1 & 0.8 & 0.1 \\
0.2725 & 0.2 & 0.7 & 0.1 \\
0.3000 & 0.3 & 0.6 & 0.1 \\
0.3225 & 0.4 & 0.5 & 0.1 \\
0.3400 & 0.5 & 0.4 & 0.1 \\
0.3525 & 0.6 & 0.3 & 0.1 \\
0.3600 & 0.1 & 0.0 & 0.9 \\
0.3600 & 0.0 & 0.2 & 0.8 \\
0.3600 & 0.0 & 0.8 & 0.2 \\
0.3600 & 0.7 & 0.2 & 0.1 \\
0.3600 & 0.9 & 0.0 & 0.1 \\
0.4125 & 0.1 & 0.7 & 0.2 \\
0.4600 & 0.2 & 0.6 & 0.2 \\
0.4725 & 0.0 & 0.3 & 0.7 \\
0.4725 & 0.0 & 0.7 & 0.3 \\
0.5025 & 0.3 & 0.5 & 0.2 \\
0.5025 & 0.1 & 0.1 & 0.8 \\
0.5400 & 0.4 & 0.4 & 0.2 \\
0.5400 & 0.0 & 0.4 & 0.6 \\
0.5400 & 0.0 & 0.6 & 0.4 \\
0.5400 & 0.1 & 0.6 & 0.3 \\
0.5625 & 0.0 & 0.5 & 0.5
\end{tabular}


Table 1 (continued).

\begin{tabular}{|c|c|c|c|c|c|c|c|c|c|c|c|}
\hline Inf & $P_{i l] \theta}$ & $P_{i 2 \mid \theta}$ & $P_{i 3 \mid \theta}$ & Inf & $P_{i l i \theta}$ & $P_{i 2 \mid \theta}$ & $P_{i 3 \mid \theta}$ & $\operatorname{Inf}$ & $P_{i J \mid \theta}$ & $P_{i 2 ; \theta}$ & $P_{i 3 \mid \theta}$ \\
\hline 0.5725 & 0.2 & 0.3 & 0.5 & 0.45 & 0.6 & 0.3 & 0.1 & 0.5725 & 0.5 & 0.3 & 0.2 \\
\hline 0.6000 & 0.2 & 0.2 & 0.6 & 0.45 & 0.1 & 0.3 & 0.6 & 0.6000 & 0.1 & 0.2 & 0.7 \\
\hline 0.6000 & 0.7 & 0.2 & 0.1 & 0.49 & 0.3 & 0.5 & 0.2 & 0.6000 & 0.6 & 0.2 & 0.2 \\
\hline 0.6025 & 0.3 & 0.5 & 0.2 & 0.49 & 0.2 & 0.5 & 0.3 & 0.6025 & 0.2 & 0.5 & 0.3 \\
\hline 0.6225 & 0.2 & 0.1 & 0.7 & 0.56 & 0.4 & 0.4 & 0.2 & 0.6225 & 0.1 & 0.5 & 0.4 \\
\hline 0.6225 & 0.4 & 0.5 & 0.1 & 0.56 & 0.2 & 0.4 & 0.4 & 0.6225 & 0.7 & 0.1 & 0.2 \\
\hline 0.6400 & 0.2 & 0.0 & 0.8 & 0.60 & 0.3 & 0.4 & 0.3 & 0.6400 & 0.2 & 0.0 & 0.8 \\
\hline 0.6525 & 0.6 & 0.3 & 0.1 & 0.61 & 0.2 & 0.3 & 0.5 & 0.6525 & 0.1 & 0.3 & 0.6 \\
\hline 0.6600 & 0.3 & 0.4 & 0.3 & 0.64 & 0.6 & 0.2 & 0.2 & 0.6600 & 0.1 & 0.4 & 0.5 \\
\hline 0.6600 & 0.5 & 0.4 & 0.1 & 0.64 & 0.2 & 0.0 & 0.8 & 0.6600 & 0.3 & 0.4 & 0.3 \\
\hline 0.7000 & 0.4 & 0.4 & 0.2 & 0.64 & 0.2 & 0.2 & 0.6 & 0.7000 & 0.2 & 0.4 & 0.4 \\
\hline 0.7125 & 0.3 & 0.3 & 0.4 & 0.64 & 0.8 & 0.0 & 0.2 & 0.7125 & 0.4 & 0.3 & 0.3 \\
\hline 0.7225 & 0.7 & 0.1 & 0.2 & 0.65 & 0.2 & 0.1 & 0.7 & 0.7225 & 0.2 & 0.1 & 0.7 \\
\hline 0.7525 & 0.5 & 0.3 & 0.2 & 0.65 & 0.7 & 0.1 & 0.2 & 0.7525 & 0.2 & 0.3 & 0.5 \\
\hline 0.7600 & 0.3 & 0.2 & 0.5 & 0.69 & 0.3 & 0.3 & 0.4 & 0.7600 & 0.5 & 0.2 & 0.3 \\
\hline 0.7600 & 0.6 & 0.2 & 0.2 & 0.69 & 0.4 & 0.3 & 0.3 & 0.7600 & 0.2 & 0.2 & 0.6 \\
\hline 0.7725 & 0.4 & 0.3 & 0.3 & 0.76 & 0.5 & 0.2 & 0.3 & 0.7725 & 0.3 & 0.3 & 0.4 \\
\hline 0.8025 & 0.3 & 0.1 & 0.6 & 0.76 & 0.3 & 0.2 & 0.5 & 0.8025 & 0.6 & 0.1 & 0.3 \\
\hline 0.8400 & 0.4 & 0.2 & 0.4 & 0.80 & 0.4 & 0.2 & 0.4 & 0.8400 & 0.4 & 0.2 & 0.4 \\
\hline 0.8400 & 0.3 & 0.0 & 0.7 & $0.8 \mathrm{I}$ & 0.6 & 0.1 & 0.3 & 0.8400 & 0.3 & 0.0 & 0.7 \\
\hline 0.8400 & 0.5 & 0.2 & 0.3 & 0.81 & 0.3 & 0.1 & 0.6 & 0.8400 & 0.3 & 0.2 & 0.5 \\
\hline 0.8400 & 0.7 & 0.0 & 0.3 & 0.84 & 0.3 & 0.0 & 0.7 & 0.8400 & 0.7 & 0.0 & 0.3 \\
\hline 0.8625 & 0.6 & 0.1 & 0.3 & 0.84 & 0.7 & 0.0 & 0.3 & 0.8625 & 0.3 & 0.1 & 0.6 \\
\hline 0.9025 & 0.4 & 0.1 & 0.5 & 0.89 & 0.4 & 0.1 & 0.5 & 0.9025 & 0.5 & 0.1 & 0.4 \\
\hline 0.9225 & 0.5 & 0.1 & 0.4 & 0.89 & 0.5 & 0.1 & 0.4 & 0.9225 & 0.4 & 0.1 & 0.5 \\
\hline 0.9600 & 0.4 & 0.0 & 0.6 & 0.96 & 0.4 & 0.0 & 0.6 & 0.9600 & 0.4 & 0.0 & 0.6 \\
\hline 0.9600 & 0.6 & 0.0 & 0.4 & 0.96 & 0.6 & 0.0 & 0.4 & 0.9600 & 0.6 & 0.0 & 0.4 \\
\hline 1,0000 & 0.5 & 0.0 & 0.5 & 1.00 & 0.5 & 0.0 & 0.5 & 1.0000 & 0.5 & 0.0 & 0.5 \\
\hline
\end{tabular}

\section{Inf}

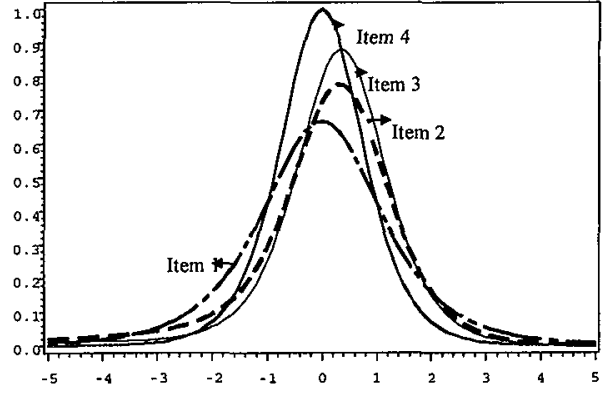

$\theta$

Figure 3. Item information functions for three items described by the 3 categories NRM. For all polytomous items $a_{i 1}=0, a_{i 3}=2$ and $c_{i g}=0$.

Note: Item 1 has $a_{12}=1.0$; Item 2 has $a_{22}=0.5$; Item 3 has $a_{32}=0.25$; Item 4 is a binary item with $a_{i}=2$ and $c_{i}=0$. $a_{i g}$ - variance Item $1<$ Item $2<$ Item $3<$ Item 4. 


\subsection{Optimal test design}

Practitioners might, at first sight, be at odds with the method that deals exclusively with conditional probabilities. Therefore, in order to provide a more tangible perspective of the polytomous/dichotomous dispute, an optimal design of a fixed-form, group-based test is carried out. The objective is to compare and find out when polytomous items might be preferred to dichotomous or vice-versa, by considering maximisation of information over a whole group of trait values simultaneously. Two common item selection criteria, known as the A- and D-optimality criteria, are applied. The item pools, from which the items are selected, are composed of both NRM and 2PL items. The effect of different groups of testtakers with different distributions of trait values is also evaluated.

\subsubsection{Procedure}

Item selection criteria. For the design of a fixed-form test, two criteria, defined as objective functions $\Phi($.$) of Fisher's Information are applied, the A- and D- optimality criteria. The A-$ optimality, trace or sum criterion, is given by

$$
\Phi\left(\operatorname{In} f_{i}\right)=\sum_{i=1}^{T} \operatorname{In} f_{i}
$$

and the product or determinant or D-optimality criterion and defined as:

$$
\Phi(\operatorname{Inf})=\prod_{i=1}^{T} I n f_{i}
$$

where $\operatorname{Inf} f_{i}$ is Fisher's information of item $i$ on a single $\theta_{l}$, as defined in (5), and (6). The index $t$ runs through the elements $\theta_{t} \in \Theta$, the vector of latent trait values representing the sample of test-takers.

The optimisation procedure selects from pool $I$ a set of $n$ items $i \in I$, for which $\Phi(\operatorname{Inf} i)$ is maximised and $n$ is the test length. For a more detailed description of optimality criteria in test design the readers are referred to Berger and van der Linden (1995), Berger and Mathijssen (1997), Berger (1998) and Lima Passos et al (2004). 
Item pools. Items were selected from two distinct pools, denoted $I$ and $J$, whose item bank functions, computed as a sum of all individual 2PL and NRM items are displayed in Figure 4. Pool $I$ is set up in such a way as to achieve a maximum overlap of dichotomous and polytomous information, having thus a greater number of dichotomous than polytomous items (600/500 with total $N=1100$ ). For item pool $J$, by contrast, this proportion is $50: 50$ with $N=1000$. Item discrimination and location parameters were randomly generated with $a_{i}, a_{i 2}$ and $a_{i 3} \sim \mathrm{U}(0.5,3)$ and $c_{i}, c_{i 2}$ and $c_{i 3} \sim \mathrm{U}(-3,3)$, with $a_{i 1}<a_{i 2}<a_{i 3}$ and $a_{i 1}=c_{i 1}=0$ for polytomous items. In order to obtain overlapping item bank information functions by dichotomous and polytomous items (Figure 4), the location parameters $c_{i}$ of dichotomous items have been slightly shifted to the left. Box-plots of the items' parametric characteristics of $I$ and $J$ can be found in Figure 5 .

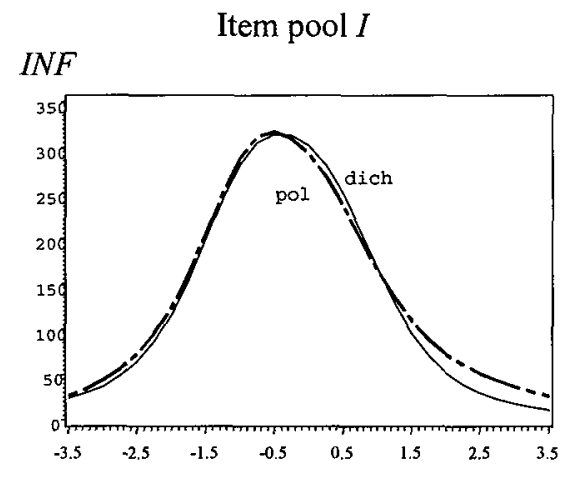

$\theta$

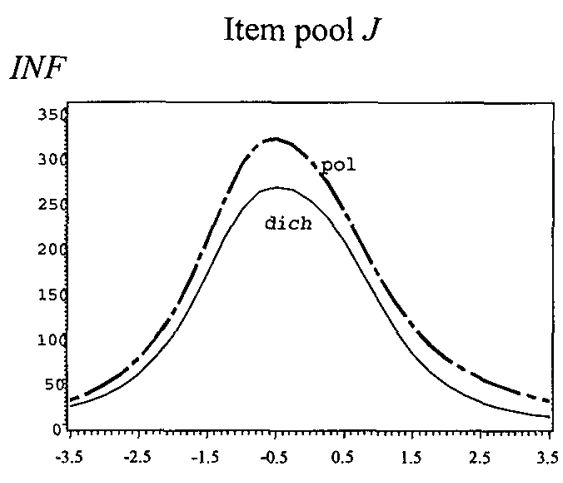

$\theta$

Figure 4. Item bank information functions - Dichotomous and polytomous contributions.

Group of examinees. $T=300$ latent trait values $\theta_{l} \in \Theta$, have been randomly generated by the standard normal and uniform distributions, $\theta_{t} \sim N(0,1)$ and $\theta_{t} \sim U(-3,3)$, resulting in $\Theta_{\mathrm{N}}$ and $\Theta_{\mathrm{U}}$, respectively. As a result, the trait values are in their majority found within the range $[-3$, 3]. Sub-samples of the examinees' have been also made by partitioning $\Theta$ in narrower ranges, with $\theta_{l} \in[-2,2], \theta_{l} \in[-1,1]$ and $\theta_{l} \in[-0.5,0.5]$. These intervals represent more homogeneous groups of examinees as regards their latent trait values. It should be noted that these intervals are symmetric around zero, with a majority of them concentrated on the central part of the latent trait scale. Interval of lower and higher trait values have been discarded due to the slight inequality between polytomous and dichotomous contributions in the total information (see Figure 4). 


\subsubsection{Results}

The proportions of selected dichotomous and polytomous items by the A- and Doptimality criteria for a fixed-form test with $n=20$ are displayed in Table 2. Given both

\section{Pool $I$ and $J$}

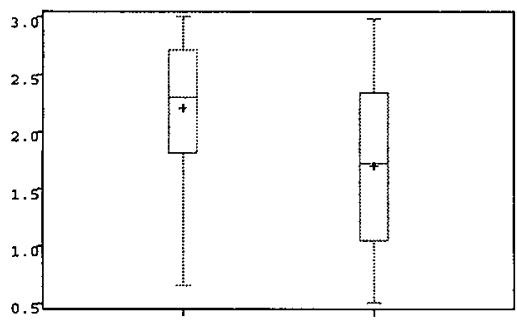

$a_{i g(\max )}-a_{i g(\min )}$

$a_{i}$

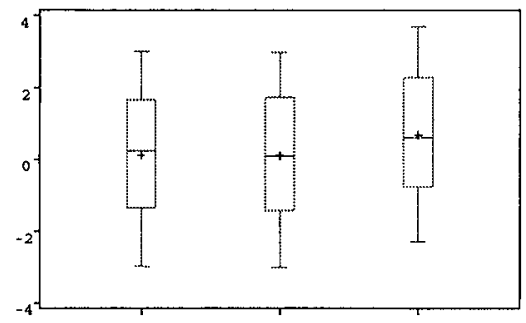

$c_{i 2}$

$c_{i 3}$

$c_{i}$

Figure 5. Box-plots for dichotomous and polytomous item parameters.

Note: For comparative purpose, the distribution of $\left[a_{i g(\max )}-a_{i g(\min )}\right]$ is displayed instead of the individual $a_{i 2}$ and $a_{i 3}$ values.

equality of information availability (Pool $I$ ) or lack thereof (Pool $J$ ), polytomous items are not given overwhelming precedence over their dichotomous items. The only exception to a balanced polytomous/dichotomous selection is given by test assemblies based on the broader trait ranges $[-3,3]$ and $[-2,2]$. Here the $D$-criterion selects exclusively (or almost) polytomous items, whereas a $50: 50$ balance is attained by the A-criterion.

\subsection{Discussion}

On the p-combinations. The positioning of dichotomous in relation to polytomous $p$ combinations in Table 1 allows for a few general conclusions to be drawn. Firstly, polytomous items described by the NRM are not necessarily advantageous in comparison to the 2PL dichotomous variant. In fact, the displayed results lend support to the idea that the opposite is closer to the truth. The validity of such statement depends on the equality conditions on the discrimination parameter values of both polytomous and dichotomous $p$ combinations, i.e. $a_{i}=\left[a_{i g(\max )}-a_{i g(\min )}\right]$.

Secondly, it has been seen that different $a_{i g}$-assemblages affect the overall realtive polytomous $p$-combinations with respect to the dichotomous ones. This result can be better 
explained by taking into account the influence different combinations of $a_{i g}$ values would have on an item's discriminating power, and more specifically on the discriminating power of its categories. An items' discriminating power is defined as the ability of an item in holding neighbouring $\theta-s$ apart, i.e. it indicates how quickly the transition from low to high probability

Table 2. Proportions of polytomous vs. dichotomous selected items $(n=20)$ by the A- and Doptimality criteria.

$\Phi$

A

$\mathrm{D}$

\begin{tabular}{|c|c|c|c|c|c|}
\hline \multirow[t]{2}{*}{$\theta$-range } & pool & $\Theta_{\mathrm{N}}$ & $\boldsymbol{\Theta}_{U}$ & $\boldsymbol{\Theta}_{\mathrm{N}}$ & $\Theta_{U}$ \\
\hline & & pol : dich & pol ; dich & pol : dich & pol : dich \\
\hline \multirow{2}{*}[-3,3]{} & $I$ & $0.45: 0.55$ & $0.45: 0.55$ & $1.0: 0.0$ & $1.0: 0.0$ \\
\hline & $J$ & $0.50: 0.50$ & $0.50: 0.50$ & $1.0: 0.0$ & $1.0: 0.0$ \\
\hline \multirow{2}{*}[-2,2]{} & $I$ & $0.50: 0.50$ & $0.45: 0.55$ & $\mathbf{0 . 8 5}: 0.15$ & $1.0: 0.0$ \\
\hline & $J$ & $0.50: 0.50$ & $0.50: 0.50$ & $0.90: 0.10$ & $1.0: 0.0$ \\
\hline \multirow{2}{*}[-1,1]{} & $I$ & $0.50: 0.50$ & $0.50: 0.50$ & $0.50: 0.50$ & $0.45: 0.55$ \\
\hline & $J$ & $0.55: 0.45$ & $0.55: 0.45$ & $0.60: 0.40$ & $0.50: 0.50$ \\
\hline \multirow{2}{*}[-0.5,0.5]{} & $I$ & $0.40: \mathbf{0 . 6 0}$ & $0.35: \mathbf{0 . 6 5}$ & $0.40: \mathbf{0 . 6 0}$ & $0.40: \mathbf{0 . 6 0}$ \\
\hline & $J$ & $0.50: 0.50$ & $0.50: 0.50$ & $0.50: 0.50$ & $0.45: 0.55$ \\
\hline
\end{tabular}

of selecting a response option takes place (Reckase \& McKinley, 1991). For dichotomous items, the absolute value of the slope parameter, $a_{i}$, is indicative of an item's discriminating power. For polytomous items, the $a_{i g}$-values alone are not sufficient to be a measure of the categories' discriminating power. Instead, the $a_{i g}$-variance was used. Of course, such claims about the discrimination parameter require the difficulty parameter to be held constant, since they also influence the shape of the category curve. In general, the higher the $a_{i g}$-variance, the more discriminative at least one of the item's categories.

Thus, polytomous items for nominal response data are not necessarily superior to dichotomous in terms of Fisher's information. Dichotomisation of polytomously scored items, 
however, may lead to loss of information on $\theta$. This loss can be explained by the changes undergone by the discrimination parameter of the monotonically increasing curve connected to the $a_{i g(\max )}$ category. After dichotomisation, the proportion of correct responses remains unaltered, while the incorrect are merged. However, the discrimination parameter of the newly turned binary item might become smaller than the discrimination parameter of the correct category in its polytomous form, so that $a_{i}<\left[a_{i g(\max )}-a_{i g(\min )}\right]$. The 'dropping' of this discrimination parameter, resulted from the merging of wrong alternatives, leads to loss of information that would have been otherwise provided by the wrong categories individually.

On the test design. The A- and D- optimality criteria are rather concordant in their proportion of selected items. A discrepancy can be found for the large trait ranges [-3,3] and [-2,2] (Table 2). This is explained by the effect the composition of the examinees' group has upon the criteria selective mechanisms. Tests assembled by the A- and D-criteria can differ considerably, the degree of which depending on the underlying trait distribution, upon which the maximisation of $\Phi$ is sought (Lima Passos et al., 2004). While the A-criterion targets primarily highly discriminative items, for both homogeneous or heterogeneous trait distributions, the D-criterion has been shown to select items with relatively lower discrimination parameters, i.e. items with broader instead of high-peak information curves, as the values of the latent traits are more diversified. For more homogenous groups of trait values, the D-criterion targets discriminative items. This explains its preference for polytomous items for the larger trait ranges $[-3,3]$ and $[-2,2]$, which stands in opposition to the tests designs for narrower ranges. Here, the polytomous/dichotomous balanced proportions for both A- and D- criteria together with the choices of the A-criterion for wider trait ranges do not support polytomous superiority of information. Similar results have been reported by Lima Passos et al. (2004) as regards different ISCs and the practical bearing of their distinct item choices. The authors conclude the A-optimality criterion to be more suitable for selecting items for cut-score, pass-fail decision tests, whereas the D-criterion should be more adequately applied for tests, whose target functions are expected to cover a broad range of trait values. Analogously, tests' targets and premises can also underpin the decision as when to use polytomous or dichotomous items, as expressed by the differentiated item selections in Table 2 . Accordingly, polytomous items, as preferred by the $D$-criterion for ranges $[-3,3]$ and $[-2,2]$, might be more suitable for tests aiming simultaneously at a diverse group of trait levels. In contradistinction, the use of dichotomous items might be especially useful when 
maximisation of information is sought over either a single trait value, or over its immediate vicinity, as it is known in tailored tests with Computerised Adaptive Testing (CAT).

Empirical studies by De Ayala (1992) and Thissen \& Steinberg (1984) with the NRM and the three parameters logistic model have shown that the enhancing impact of NRM on the $\hat{\theta}$-precision was only found at the lower level of the $\theta$-range. So far, the possibility that polytomous items estimate high $\theta$-levels with greater precision than dichotomous has not found any endorsement in the practical world. It has been argued that for high $\theta$-levels, at which the test-takers are bound to choose the correct answer, the utility of a differentiated choice of incorrect responses becomes less meaningful. Furthermore, a study on CAT tests composed by NRM items showed that they might reach the same accuracy as dichotomous tests with less number of administered items (De Ayala, 1992). To this latter finding a concluding remark might me worthwhile. The more the item parameters, the larger the sample of test-takers required for their calibration. Thus, one should ponder whether the final gain in terms of fewer items pays off the process of estimating several item parameters (NRM), instead of only two/three (2PL, 3PL). On the evidence of this study, a cost-benefit evaluation might not necessarily favour polytomous items for CAT tests with the nominal response format. 
Chapter 6 
Epilogue

7 Epilogue 
Chapter 7 


\section{Epilogue}

Since Fisher's notorious works on statistical methods for researchers and design of experiments, firstly published in 1925 and 1935, respectively (Fisher, 1990), the world of experimental research has been dominated by the statistical principles of optimal design. This approach aims at the most efficient allocation of observations units, in order to obtain good estimate of effect and/or error variances, given financial, time or any other practical constraint.

Compared to natural and engineering sciences, the impact of optimal experimental design on psychological, educational and social sciences research has been relatively less pervasive. This can be partly accounted for by the high level of complexity and technicality of the available literature optimal design (McClelland, 1997). But, as the latter author claims and the increasing amount of publications show, the application of optimal design in psychological and social sciences has come of age.

One way of bringing the theory of design optimisation within the grasp of a larger audience is by accentuating the practical relevance and conceptual understanding of its mathematical building blocks. By means of an example, McClelland says that, "without pursuing the technicalities of optimal design, psychologists can greatly improve the efficiency of their research designs simply by considering the variance of their independent variables. Maximising the variance of independent variables improves efficiency and statistical power" (McClelland, 1997, p. 4). Following this concept-driven approach, the present work has attempted to refrain from heavy technical and mathematical elaborations, without, however undermining the accuracy of its content. The idea was to find a balance between technical details and practice oriented strategies. Conceptual aspects of the applied techniques have been highlighted and the practical implications of the obtained results discussed in each of the independent, self-contained chapters.

In this final session a concise overview of the research results is offered. The issues of sampling (chapter 2) and test designs (chapter 3 to 6) are handled separately. The main conclusions are condensed and a gist of the thesis' main messages presented. Concurrently, shortcomings of the applied techniques are discussed and suggestions for further research made. 


\subsection{Optimal sampling designs}

As put forward by Buyske (2004), one can expect optimal design to remain the major approach for handling the issue of item parameters calibration. Chapter 2 shows maximin designs based on the D-optimality criterion to be useful for circumventing the problem of local optimality. In association with the concept of relative efficiency maximin designs guarantee the least possible loss in efficiency. The degree of efficiency loss is determined by the parametric diversity of the set of items to be calibrated (item space). It has been demonstrated that the greater the parametric diversity in the items spaces, the greater the efficiency loss. This applies to both locally optimal and alternative calibration designs. Among these, the uniform distribution of latent traits showed comparable performances to maximin designs, manifesting greater robustness against efficiency loss than other alternative designs as the random seeding. Even by considering that maximin designs might not be easily implemented in practice, they remain a quantitative basis, upon which the selection of an appropriate design can be based. Although this study was based on the NRM, the applied method can be extended to other polytomous IRT without any reservation.

\subsection{Optimal test designs}

Contrary to calibration issues, techniques of design optimisation are less frequently applied to test construction. Possibly the presence of alternative methods for optimal test assembly that explicitly accommodate practical constraints account to a great extent for the relative low profile of optimal test designs. However, what is easily overlooked is the fact that, within the optimal design framework, sequential algorithms can easily incorporate practical constraints, as suggested in chapter 3. These constraints have been, however, not considered herein. Disregarding practical test constraints might at first negatively affect any research's validation and acceptance. This can be, however, justified.

Previous study has demonstrated that A- and D- optimality criteria can substantially diverge in their item selection (Berger and van der Linden, 1995). Less clear were the reasons underlying the reported differences. It has been a task of this research to shed some light on this topic, by investigating and explaining the criteria's choices in basic and simple terms, given different test settings (chapters 3 to 6 ).

It is well known that test constraints have a restrictive effect on item selection. This means that they can alter the criteria primary choices, possibly obscuring the reasons for the criteria distinct item selections. Putting test constraints aside allowed for an understanding of 
the criteria selective mechanisms (chapter 3) as a function of the item pool and trait distribution. This understanding enables a criterion to be picked that best meets a test's specific target information function. Needless to say, the next step would be an investigation of how and to what extent the observed patterns of item selection are altered once item selection is subject to constraints.

\subsubsection{Optimality criteria}

The research focused on three criteria, two variants of Fisher's Information, the Aand D-optimality criteria, and the global information criterion. The A- and D-criteria have been specifically chosen because they convey a summary measure along a trait range or distribution via summation or product of local information values. The present methods can be easily extended to include other objective functions of Fisher's information known in the literature of optimum experimental design (Atkinson and Donev, 1996). It remains to be seen whether other available criteria and their selective preferences can be used in a context of objective-driven test construction, as it has been shown for the A- and D-criteria.

A-criterion. For both fixed-form (chapter 3 ) as well as adaptive tests (chapters 4 and 5), the A-criterion is shown to prioritise discriminative items, leading to target information functions (TIFs) that offer high information peaks, but cover just a relative small range of trait values. The A-criterion is hence suitable for $\mathrm{p} \& \mathrm{p}$ certification, cut-off score or criterion-referenced tests, in which maximum information for a threshold value on the latent trait continuum is sought (Wiberg, 2003). The A-criterion might be less desirable in CAT early stage. The choice of discriminative items in this phase enhances the risk of estimation instability. However, as it is shown in both CAT chapters (4 and 5), estimation accuracy, precision and stability depend in first instance on the information quality of the item pool, and secondarily on the items selection criterion. High parametric diversity, resulting in information more uniformly spread over the traits, enhances the quality of estimates, irrespective of the applied criterion.

KL-criterion. The results of chapter 4 and 5 support to some extent $\mathrm{KL}$ or global information criterion's robustness against CAT estimation instability, as advocated by Chang and Ying's (1996). For large trait intervals in CAT early stage, KL criterion's parametric preferences are similar to the A-criterion, targeting discriminative items. Contrary to the latter, it manages to recover more quickly from any initial bias and avoid recurring ones as the test progresses. As 
a whole, the global information criterion remains recommendable. A single reservation would be its more sophisticated and numerical handling, requiring long computational time. This property, however, becomes irrelevant with increasing speed of modern computing.

D-criterion. The application of the $\mathrm{D}$-criterion as a viable item selection procedure for CAT was motivated by its greater sensitivity to the underlying trait distribution, demonstrated for fixed-form tests (chapter 3 ). The final TIFs of $p \& p$ tests selected by the D-criterion over a large trait interval are comparatively low and wide, but higher and narrow, as the width of the trait interval is reduced. For group-based examinations this criterion would be thus more adequately applied to aptitude tests. In a CAT setting (Chapter 4 and 5) this same flexible pattern of item selection was observed. The D-criterion started with relative low discriminating items (wider TIFs) in CAT early stage, switching to higher ones, as the trait confidence intervals narrowed. It is believed that the key to its early stage relative stability lies precisely in the initial selection of items with wider instead of higher TIFs, circumventing in this way the attenuation problem. The D-criterion is shown to be a feasible and recommendable item selection procedure for CAT.

The criteria item exposure rates. No measures for controlling item exposure were applied. The three criteria exposure rates were evaluated and compared in their raw form (chapter 5). Preliminary results demonstrated that a uniform usage of items in a pool is difficult to attain without the intervention of a proper controlling measure. This is valid for all three criteria. In spite of the fact that a greater flexibility of item choices could be slightly detected for the Dand KL- criteria, a more relevant result indicated item usage to be primarily influenced by the item pool composition. A larger pool with greater parametric diversity led to similar criteria performances, resulting in overlapping exposure rates among them. The nuances related to the effect of the item pool composition on the item exposure rates and to whether the estimation performances of different criteria are equally affected by controlling measures are subject for future investigation.

\subsubsection{Polytomous vs. dichotomous information debate}

The thesis is closed with a chapter that explores the information debate around polytomous and dichotomous items. Chapter 6 was intended to lay bare inconsistencies that can be found in the literature on this subject matter and offer some consensus. In the centre of this debate stood items with a nominal response format, since graded response items offer 
unequivocally more information on the latent trait than dichotomous items. As throughout the chapters dealing with test design optimisation, the question of which item to use, polytomous or dichotomous, is better answered by taking into account a test's target information functions.

Given due consideration to location parameters and equality of discriminating power, polytomous items usually offer wider information functions but with lower maxima than dichotomous items. Therefore, the latter can be expected to estimate a single trait as efficiently as the former, if not better. The situation changes once a wide interval or distribution of heterogeneous trait values is at stake. In this case, polytomous items are bound to offer some advantage due to the wider tails of their information functions. This explains why in comparative studies with $\mathrm{p} \& \mathrm{p}$ tests, polytomous items seem to increase measurement precision only for lower trait values (Thissen and Steinberg, 1984; De Ayala, 1992). Possibly here lies also the reason why in adaptive tests, polytomous CAT with NRM have been reported to have reached the same accuracy as dichotomous with less number of items administered (De Ayala, 1992). As shown in previous chapters, items with broader information functions are less likely to generate instability during early stage. As a result, the estimate's convergence to the true trait value is swifter, affecting in turn the test length. In spite of these advantages, a test maker is advised to ponder on pros and contras, before opting for polytomous items in CAT tests. The calibration of those items might require considerably larger samples of test-takers. The extra calibration costs might not be compensated by the reduction of test length. A careful investigation of this issue with precise cost-benefit evaluation is required. 
Chapter 7 


\section{References}

Abrahamowicz, M. and Ramsay, J.O. (1992). Multicategorical spline models for item response theory. Psychometrika, 57, 5-27.

Andrich, D. (1978). A rating scale formulation for ordered response categories. Psychometrika, 43, 561-573.

Armstrong, R.D., Jones, D.H. and Kunce, C.S. (1998). IRT test assembly using network-flow programming. Applied Psychological Measurement, 22,237-247.

Atkinson,A.C., and Donev, A.N. (1996). Optimum experimental designs. Oxford: Clarenden Press.

Baker, Frank B. (1992). Item response theory: parameter estimation techniques. New York, Basel, Hong Kong: Marcel Dekker, Inc.

Berger, M.P.F. (1991). On the efficiency of IRT models when applied to different sampling designs. Applied Psychological Measurement, 15, 283-306.

Berger, M.P.F. (1992). Sequential sampling designs for the two-parameter item response theory model. Psychometrika, 57, 521-538.

Berger, M.P.F. (1994a). D-optimal designs for item response theory models. Journal of Educational Statistics, 19, 43-56.

Berger, M.P.F. (1994b). A general approach to algorithmic design of fixed-form tests, adaptive tests and testlets. Applied Psychological Measurement, 18, 141-153.

Berger, M.P.F. and van der Linden, W.J. (1995). Ontwerpen van tests met verschillende optimaliteitscriteria [Designing educational tests with different criteria of optimality]. Tijdschrift voor Onderwijsresearch, 20, 79-92.

Berger, M.P.F. and Veerkamp, W.J.J. (1996). A review of selection methods for optimal tests design. In M. Wilson and G. Engelhard (Eds.), Objective Measurement: Theory into practice (Vol. 3, pp.437-455). Norwood NJ: Ablex.

Berger, M.P.F. and Mathijssen, E. (1997). Optimal test designs for polytomously scored items. British Journal of Mathematical and Statistical Psychology, 50, 127-141.

Berger, M.P.F. (1998). Optimal design of test with dichotomous and polytomous items. Applied Psychological Measurement, 22, 248-258.

Berger, M.P.F, King, C.Y.J., and Wong, W.K. (2000). Minimax D-optimal designs for item response theory models. Psychometrika, 65-3, 377-390.

Bock, R.D. (1972). Estimating item parameters and latent ability when responses are scored in two or more nominal categories. Psychometrika, 37, 29-51. 
Buyske, S.G. (1998). Optimal design for item calibration in computerized adaptive testing. Unpublished doctoral dissertation. New Brunswick, New Jersey.

Buyske, S. (2003). Optimal design in educational testing. In M. P.F. Berger and W.K.Wong (Eds.), Applied optimal designs. New York: Wiley. In Press.

Chang, H. and Ying, Z. (1996). A global information approach to computerized adaptive testing. Applied Psychological Measurement, 20, 213-229.

Chang, H. and Ying, Z. (1999). a-stratified multistage computerized adaptive testing. Applied Psychological Measurement, 23, 211-222.

Chang, H. and van der Linden, W.J. (2003). Optimal stratification of item pools in $a$ stratified computerized adaptive testing. Applied Psychological Measurement, 27, $262-274$

Chen, S.Y., Ankenmann, R.D. and Chang, H.H. (2000). A comparison of item selection rules at the early stages of computerized adaptive testing. Applied Psychological Measurement, 24, 241-255.

Cheng, P.E. and Liou, M. (2000). Estimation of trait level in computerized adaptive testing. Applied Psychological Measurement, 24, 257-265.

Cheng, P.E. and Liou, M. (2003). Computerized adaptive testing using the NearestNeighbors criterion. Applied Psychological Measurement, 27, 204-216.

De Ayala, R.J. (1992). The nominal response model in computerized adaptive testing. Applied Psychological Measurement, 16, 327-343.

De Ayala, R.J. (1993). An introduction to polytomous item response theory models. Measurement and Evaluation in Counseling and Development, 25,172-189.

Dodd, B.G., De Ayala, R.J., and Koch, W.R. (1995). Computerized adaptive testing with polytomous Items. Applied Psychological Measurement, 19, 5-22.

Drasgow, Fritz (1995). Introduction to the polytomous IRT special issue. Applied Psychological Measurement, 19, 1-3.

Eggen, T.J.H.M. (1999). Item selection in adaptive testing with the sequential probability ratio test. Applied Psychological Measurement, 23, 249-261.

Everitt, B.S. (1998). The Cambridge Dictionary of Statistics. Cambridge:Cambridge University Press.

Fan, M. and Hsu, Y. (1996, April). Utility of Fisher information, global information and different starting abilities in mini CAT. Paper presented at the Annual Meeting of the National Council on Measurement in Education, New York. 
Fisher, R.A (1990). Statistical Methods, Experimental Design, and Scientific Inference - A Re-issue of Statistical Methods for Research Workers, The Design of Experiments and Statistical Methods and Scientific Inference. Edited by J.H. Bennet. Oxford: Oxford University Press.

Hambleton, R.K. and Swaminathan, H. (1985). Fundamentals of Item Response Theory. Newburry Park, London, New Delhi: Sage Publications.

Hambleton, R.K., Swaminathan, H. and Rogers. J. (1991). Item Response Theory Principles and Applications. Boston, Dordrecht, Lancaster: Kluwer Nijhoff Publishing.

Hambleton, R.K. and Jones, R.W. (1993). Comparison of classical test theory and item response theory and their applications to test development. Educational Measurement: Issues and Practice, 12(3), 38-47.

Heinen, A.G.J.J. (1996). Latent class and discrete latent trait models: similarities and differences. London: Sage Publications.

Holman, R., and Berger, M.P.F. (2001). Optimal calibration designs for tests of polytomously scored items described by item response theory models. Journal of Educational and Behavioral Statistics, 26, 361-381.

Imbos, Tj., Does, R.J.M.M., Janssen, M.P.E., Tan, F.E.S. and Verwijnen, G.M. (1990). A statistical model for the measurement of growth in medical knowledge. In Bender, W, Hiemstra, R.J., Scherpbier, A.J.J.A. and Zwiestra, R.P. (Eds.). Teaching and assessing clinical competence. Boekwerk, Groningen (pp.219-226).

Jones, D.H., and Jin, Z. (1994). Optimal sequential designs for on-line item estimation. Psychometrika, 59, 59-75.

Jones, D.H., and Nediak, M.S. (2000). A Simulation study of optimal on-line calibration of testlets using real data. RUTCOR research report, Rutgers the State University of New Jersey: Faculty of Management and RUTCOR.

Lord, F.M. and Novick, M.R. (1968). Statistical theories of mental test scores. Reading, MA: Addison Wesley.

Lima Passos, V., Berger, M.P.F. Muijtjens, A.M.M. and Tan, F.E.S. (2003). Test Design Optimisation for the Nominal Response Model. (submitted paper).

Lima Passos, V., Berger, M.P.F. and Tan, F.E.S. (2004). Test Design in CAT early stage with the Nominal Response Model. (submitted paper).

Lima Passos, V. and Berger, M.P.F. (2004). Maximin calibration designs for the Nominal Response Model: An empirical evaluation. Applied Psychological Measurement, 28, $72-87$. 
Luecht, R.M. (1998). Computer-assisted test assembly using optimization heuristics. Applied Psychological Measurement, 22, 224-236.

Masters, G.N. (1982). A Rasch model for partial credit scoring. Psychometrika, 47, 149-174.

Mellenbergh, G.J. (1995). Conceptual notes on models for discrete polytomous item responses. Applied Psychological Measurement, 19, 91-100.

McClelland, G.H. (1997). Optimal design in psychological research. Psychological Methods, 2, 3-19.

Nedelsky, L. (1954). Ability to avoid gross error as a measure of achievement. Educational Psychological Measurement, 14, 459-472.

Owen, R.J. (1975). A Bayesian sequential procedure for quantal response in the context of adaptive mental testing. Journal of the American Statistical Association, 70, 351-356.

Ouwens, J.N.M., Tan, Frans E. S. and Berger, M.P.F. (2002). Maximin D-optimal designs for longitudinal mixed effects models. Biometrics, 58(4), 735-741.

Reckase, M.D. and Mckinley, R.L. (1991). The discriminating power of items that measure more than one dimension. Applied Psychological Measurement, 15, 361-373

Samejima, F. (1969). Estimation of latent ability using a response pattern of graded scores. Psychometrika Monograph Supplement, No 17.

Samejima, F. (1977). A use of the information function in tailored testing. Applied Psychological Measurement, 1, 233-247.

Sanders, P.F. and Verschoor, A.J. (1998). Parallel test construction using classical item parameters. Applied Psychological Measurement, 22, 212-223.

Stocking, M.L. (1990). Specifying optimum examinees for item parameter estimation in Item Response theory. Psychometrika, 55, 461-475.

Tan, F.E.S., Imbos Tj., Does, R.J.M. (1994). A distribution free approach for comparing growth of knowledge. Journal of Educational Measurement, 31, 51-65.

Tan, F.E.S., Imbos Tj., Does, R.J.M., and Theunissen, M. (1995). Optimal unbiased classification rule for mastery testing based on longitudinal data. Educational and Psychological Measurement, 55, 595-613.

Tatsuoka, K.K. (1983). Rule space: An approach for dealing with misconceptions based on item response theory. Journal of Educational Measurement, 20, 345-354.

Thissen, D. (1976). Information in wrong responses to the Raven progressive matrices. Journal of Educational Psychology, 13, 201-214. 
Thissen, D., and Steinberg, L. (1984). A response model for multiple choice items. Psychometrika, 49, 501-519.

Thissen, D., and Steinberg, L. (1986). Taxonomy of item response models. Psychometrika, 51, 567-577.

Thissen, D., Steinberg, L. And Fitzpatrick, A.R. (1989). Multiple-choice models: The distractors are also part of the item. Journal of Educational Measurement, 26, 161-176.

Thissen, D. (1991). MULTILOG, Version 6.0. Chicago: Scientific Software Inc.

van der Linden, W.J. (1994). Optimum design in item response theory: Test assembly and I tem calibration. In G.H. Fisher and D. Laming Contributions to mathematical psychology, psychometrics and methodology (pp. 305-318). New York, NY: Springer Verlag.

van der Linden, W.J. and Hambleton, R.K., eds. (1997). Handbook of Modern Item Response Theory. New York Berlin: Springer Verlag.

van der Linden, W.J. (1998). Optimal assembly of psychological and educational tests. Applied Psychological Measurement, 22, 195-211.

van der Linden, W.J. (1998). Bayesian item-selection criteria for adaptive testing. Psychometrika, 62: 201-216.

van der Linden, W.J. and Reese, L.M. (1998). A model for optimal constrained adaptive testing. Applied Psychological Measurement, 22,259-270.

van der Linden, W.J. and Pashley, P.J. (2000). Item selection and $\theta$ estimation in adaptive testing. In W.J. van der Linden and C.A.W. Glas (eds.) Computerized Adaptive Testing: Theory and Practice (pp.1-25). Dordrecht/Boston/London: Kluwer Academic Publishers.

van der Linden, W.J. and Glas, C.A.W. (2000). Computerized Adaptive Testing: Theory and Practice (pp.1-25). Dordrecht/Boston/London: Kluwer Academic Publishers.

van der Vleuten, C.P.M, Verwijnen, G.M and Wijnen, W.H.F.W. (1996). Fifteen years with progress testing in a problem-based learning curriculum. Medical Teacher, 18, 103109.

van Rijn, P.W., Eggen, T.J.H.M., Hemker, B.T. and Sanders, P.F. (2002). Evaluation of selection procedures for computerized adaptive testing with polytomous items. Applied Psychological Measurement, 26, 393-411.

Veldkamp, P. B. (2001). Principles and Methods of constrained Test Assembly. Doctoral dissertation, The University of Twente, The Netherlands. 
Veldkamp, B.P. (2003). Item selection in polytomous CAT. In: H.Yanai, A. Okada, K. Shigemasu, Y. Kano, \& J.J. Meulman (Eds.). New Developments in Psychometrics (pp.207-214). Tokyo, Japan: Springer Verlag.

Veerkamp, W.J.J. and Berger, M.P.F. (1997). Some new item selection criteria for adaptive testing. Journal of Educational and Behavioral Statistics, 22:203-226.

Vorst, H.C.M. (2000). Schoolvragenlijst (School Attitude Questionnaire). Lisse, The Netherlands: Swets \& Zeitlinger.

Weissman, A. (2003). Information theoretic approaches to item selection. Paper presented at the International meeting of the Pshychometric Society, (IMPS), Italy.

Wiberg, M. (2003). An Optimal Design approach to criterion-referenced computerized testing. Journal of Educational and Behavioral Statistics, 28, 97-110.

Wynn, H.P. (1970). The sequential generation of D-optimum experimental designs. Annals of Mathematical Statistics, 41, 1655-1664.

Yi, Q. and Chang, H.H. (2003). a-Stratified CAT design with content blocking. British Journal of Mathematical and Statistical Psychology, 56, 359-378. 


\section{Summary}

The objective of this thesis is to apply statistical methods of design optimisation to improve estimation of parameters of polytomous Item Response Theory (IRT) models. For these models, no global optimal designs can be found, i.e. optimal, irrespective of the values the model parameters might take. The reason for that lies in the dependence of non-linear models' information matrices on the unknown parameter values. Local optimality, i.e. designs that are optimal for a given set of parameters, can be found but usually offers a spurious solution to the design problem. Throughout this thesis a pre-specification of a parameters' range is used to handle the lack of knowledge of the parameters values.

Chapter one is introductory. It describes two polytomous IRT models to be dealt with in this thesis: the Nominal Response Model (NRM) and the Graded Response Model (GRM). The core of statistical techniques of design optimisation within the context of Item Response Theory (IRT) is shortly presented. In IRT, the issue of design optimisation is usually differentiated in two cases: optimal sampling or calibration design and optimal test design. In the former an optimal sample of test-takers is sought over a parameters space of latent trait values to efficiently estimate the item parameters. In optimal test design, which aims at a precise and accurate estimation of multiple or single trait values by selecting the set of most informative items from an item pool (item parameters space), a further differentiation between group-based and individual trait estimation can be made.

Chapter two deals with optimal calibration of items described by the NRM. An approach based on the concept of relative efficiency within a maximin framework is implemented by means of the D-optimality criterion. By varying the composition of the parameter space, it is shown that the higher the parametric diversity of the item space, the greater the efficiency loss of the maximin design. Alternative designs, like designs with a uniform and normal distribution of latent trait values also display this effect. With relative efficiencies often comparable to the ones of maximin designs, the uniform distribution emerges as a robust design against efficiency loss. Maximin designs offer a quantitative basis, upon which solid comparisons among different sampling designs can be drawn.

Chapter three uses the optimal design approach to select NRM items for a groupbased, fixed-form test. Two objective functions of Fisher's Information, the A- and Doptimality criteria are applied and their selective strategies investigated. The criteria final test functions are evaluated as a function of varied latent trait distributions and item pool compositions. The distinct test assembly by the different criteria raises the possibility of 
matching the item selection criterion to the specific test goals, usually expressed in form of a target information function. The obtained results suggest a greater sensitivity of the Doptimality criterion to changes of the underlying trait distribution. The A-criterion, by contrast, seems to manifest a clear preference for highly discriminative items, irrespective of the trait distribution. Due to the more accentuated dependence on the trait distribution of the item selection by the D-criterion, its suitability for computerised adaptive testing (CAT) is anticipated.

Chapter four evaluates the performance of the D-optimality criterion in CAT, demonstrating its feasibility for individually tailored tests. Two other criteria, A-optimality the Kullback-Leibler (KL) information criteria, are also considered for comparative purposes. A CAT simulation study is carried out, giving special emphasis to the initial phase of trait estimation. The so-called CAT early stage can be characterised by instability of the trait estimates. It is known that the estimates in this stage need to be corrected for bias. In general, this study shows that the spread of item bank information along the latent trait continuum is a major factor determining accuracy, precision and stability of trait estimation. The more uniform this information spread, the greater the quality of estimation for all applied criteria. For less uniformly spread information, all criteria perform similarly in terms of measurement accuracy and precision at the end of the test. They, however, differ in estimation stability during the early stage. The A-criterion displayed accentuated fluctuations of mean squared errors (MSE), while the D- and KL-criteria seemed to circumvent the attenuation paradox more effectively. Due to its flexibility of item selection, switching between low and high discriminative items as the test unfolds, it is conjectured that the D-criterion could make a more balanced use of the available items in a pool.

Chapter five extends the same methods from chapter four to an adaptive test with the GRM with reproducible results, including the robustness of the D-optimality criterion against early stage instability. The criteria preferences with respect to the parameter characteristics of the selected items are more closely investigated as an underpinning explanation for the criteria performances. The additional issue of item exposure is also evaluated. No measure for controlling the criteria item exposure rates is applied. The foregoing assumption as regards D-criterion's balanced use of items in a pool (chapter 4) could not be fully confirmed. In the matter of item pool usage, the larger the pool (with higher parametric diversity) the more overlapping the exposure rates of the three applied criteria.

In literature references there is a lack of congruity among opinions as regards which sort of items, polytomous or dichotomous, supply more information on the unknown latent trait. Generally, it is taken for granted that polytomous items are more informative than 
dichotomous. While this assertion is true for polytomous items described by the GRM, its validity is questionable for items with a nominal response format. Chapter six shows through a polytomous-dichotomous transition of the NRM that, given due consideration to item discrimination parameters, dichotomous items can be as informative as their polytomous counterpart, if not more. The findings of test design optimisation, in which items are selected from a composite pool with polytomous NRM and dichotomous 2PL items, supports this statement and shows that the general view favouring polytomous items is not always justified for the NRM. The test' target information function and the test's setting (paper and pencil or adaptive) are major factors to be considered in the choice between dichotomous and polytomous items. It is argued that the gain in measurement precision, that might be achieved through polytomous items, could be traded-off for the parametric simplicity of dichotomous items. 


\section{Samenvatting}

Het doel van dit proefschrift is om statistische methoden voor design optimalisatie te gebruiken voor efficiënte schatting van parameters in polytome Item-Respons-Theorie (IRT) modellen. Voor deze modellen kunnen geen globale optimal designs worden gevonden, d.w.z. optimaal voor alle mogelijke waarden die de model parameters zouden kunnen aannemen. De reden daarvoor ligt in de afhankelijkheid van de informatiematrices bij nietlineaire modellen van de onbekende parameters. Lokale optimaliteit, d.w.z. designs die optimaal zijn voor een gegeven set van parameter waarden, kunnen gevonden worden maar bieden gewoonlijk een niet juiste oplossing voor het design probleem. In dit proefschrift wordt voornamelijk aan de hand van een gespecificeerde design de informatie over de parameters gemaximaliseerd.

Hoofdstuk een is inleidend. Het beschrijft de twee polytome IRT modellen waarmee in dit proefschrift gewerkt wordt: het Nominale Responsmodel (NRM) en het Graded Responsmodel (GRM). De belangrijkste statistische technieken voor design optimalisatie binnen de context van de Item-Respons-Theorie (IRT) worden in het kort gepresenteerd. In IRT wordt design optimalisatie gewoonlijk onderscheiden in twee gevallen: optimale steekproef of calibratie design en optimale test design. In het eerste geval wordt een optimale steekproef van test-nemers gezocht om de item parameters zo efficiënt mogelijk te schatten. In optimaal test design, dat zich richt op een nauwkeurige en accurate schatting van de latente kenmerken door de meest informatieve verzameling van items uit een item 'pool' (item parameter ruimte) te selecteren, kan een verdere differentiatie gemaakt worden tussen groepgewijze en individuele schattingen van latente kenmerken.

Hoofdstuk twee behandelt optimale calibratie van items beschreven door het NRM. Een benadering gebaseerd op het concept van relatieve efficiëntie binnen een maximin kader d.m.v. het D-optimaliteitscriterium wordt toegepast. Door de samenstelling van de parameter ruimte te variëren wordt aangetoond dat hoe groter de spreiding van de items parameter waarden in de item-ruimte is, des te groter het verlies aan efficiëntie van het maximin design. Ook alternatieve designs, met een uniforme en normale verdeling van de waarden van de latente kenmerken vertonen dit effect. Het uniforme design heeft vaak vergelijkbare relatieve efficiëncies als het maximin design. Maximin designs bieden een kwantitatieve basis voor een zinvolle vergelijking tussen verschillende calibratiedesigns.

Hoofdstuk drie gebruikt de optimal design benadering om NRM items te kiezen voor een 'fixed-form' toets. Twee doelfuncties van Fisher's informatie, de A- en D- optimaliteits- 
criteria worden toegepast en hun selectie mechanismen onderzocht. De testinformatiefuncties worden geëvalueerd als een functie van verdelingen van latente kenmerken en de itempool samenstelling. De afzonderlijke testsamenstellingen van de verschillende criteria biedt de mogelijkheid om het itemselectiecriterium af te stemmen op het specifieke doel van de test. De verkregen resultaten suggereren een grotere gevoeligheid van het $\mathrm{D}$-optimaliteitscriterium voor verandering van de verdeling van de latente kenmerken. Het A-criterium lijkt daarentegen een duidelijke voorkeur voor zeer discriminative items te vertonen ongeacht de verdeling van de latente kenmerken. Wegens de gevoeligheid van het $\mathrm{D}$-criterium voor de verdeling van latente kenmerken, wordt verwacht dat dit criterium geschikt is voor computerised adaptive testing (CAT).

Hoofdstuk vier evalueert de prestatie van het D-optimaliteitscriterium in CAT, en demonstreert de toepasbaarheid ervan. Twee andere criteria, het A-optimaliteit en het Kullback-Leibler $(\mathrm{KL})$ informatie criterium, worden eveneens onderzocht. Een CAT simulatie studie is uitgevoerd waarbij nadruk gelegd wordt op de 'early stage' schattingen van latente kenmerken. De zogenaamde 'CAT early stage' wordt meestal gekarakteriseerd door instabiliteit van de parameterschattingen. Het is bekend dat in dit stadium de schattingen gecorrigeerd moeten worden voor bias. In het algemeen laat deze studie zien dat de spreiding van itembankinformatie over het continuüm van de latente kenmerken de voornaamste factor is in het bepalen van de nauwkeurigheid, precisie en stabiliteit van de schatting van latente kenmerken. Des te meer uniform deze spreiding van informatie is, des te groter is de kwaliteit van de schatting voor alle gebruikte criteria. Voor niet uniform verdeelde informatie presteren alle criteria ongeveer gelijk voor wat betreft meet-nauwkeurigheid en precisie aan het eind van de test. Ze verschillen echter in stabiliteit van de schattingen in de 'early stage'. Het Acriterium laat nadrukkelijke fluctuaties zien in de mean squared error (MSE) terwijl de D- en KL-criteria de attenuatie paradox effectiever lijken te omzeilen. Door de flexibiliteit van het D-optimaliteitscriterium bij het selecteren van items, waarbij afwisselend tussen hoge en lage discriminerende items wordt gekozen, lijkt het D-criterium een meer evenwichtig gebruik te maken van de beschikbare items in de pool.

Hoofdstuk vijf breidt dezelfde methoden van hoofdstuk vier uit naar een adaptive test met het GRM en geeft vergelijkbare resultaten m.b.t de robuustheid van het Doptimaliteitscriterium tegen instabiliteit in 'CAT early stage'. De specifieke selectie van itemparameter waarden door de criteria zijn nader onderzocht. Het probleem van 'item exposure' is ook geëvalueerd. Het resultaat dat het D-criterium een meer gebalanceerde gebruik van items in een pool maakt kon niet ten volle worden bevestigd. Wat 'item 
exposure' betreft, hoe grote de itempool (met grotere verschillen tussen parameter waarden) des te meer overlap in exposure frequencies tussen de drie gebruikte criteria.

In de literatuur is weinig overeenstemming tussen opinies over welk soort items, polytome of dichotome, meer informatie over de onbekende latente kenmerken leveren. In het algemeen wordt aangenomen dat polytome items meer informatief zijn dan dichotome. Hoewel deze aanname waar is voor polytome items die door het GRM worden beschreven, is deze aanname niet altijd geldig zijn voor items met een nominale response format. Hoofdstuk zes laat voor het NRM zien dat, als men corrigeert voor de itemdiscriminatie parameters, de dichtome items even informatief kunnen zijn als hun polytome tegenhangers, zo niet meer. De bevindingen van test design optimalisatie waarin de items worden geselecteerd uit een samengestelde pool met polytome NRM en dichotome 2PL items, ondersteunt dit resultaat en laat zien dat het standpunt dat polytome items meer informatie leveren niet altijd gerechtvaardigd is. De testinformatie functie en de vorm van de test (paper\&pencil tegenover adaptief) zijn de belangrijkste factoren bij de keuze tussen dichotome en polytome items. Er wordt geconcludeerd dat de winst in nauwkeurigheid die bereikt kan worden door polytome items, ingeruild zou kunnen worden voor de parametrische eenvoud van dichotome items.

Acknowledgment: I wish to gratefully acknowledge Marion de Leeuw's help, without which I would be literarily 'Lost in Translation'. 


\section{Curriculum Vitae}

Valeria Lima Passos was born in Salvador, a tropical city on the northeast coast of Brazil, in March 1965. After finishing her graduation on Oceanology in Rio Grande do Sul (1986), South-Brazil, she came to Europe for a German language course. After her second graduation in Biology (1992) from the Freie Universitaet Berlin, she obtained an MPhil in History and Philosophy of Science at Queen's University, in Belfast, Northern Ireland. Her varied academic interests stayed in the way of a premature specialisation. This was changed by Caio's arrival, her son, prompting her to willingly settle for biometrics in 1998, after a specialisation in Tuebingen, Germany. Since then she has been involved with applied statistics, working in the department of Medical Statistics at the University of Edinburgh and Medical Research Council (Human Genetics Unit), also in Edinburgh, Scotland. In 2000 she was accepted as a PhD student (AIO) in the department of Methodology and Statistics, at the University of Maastricht, the Netherlands. In September 2004 she started working as a postdoc in the same department. 


\section{Post-scriptum (personal acknowledgements)}

For this work the merits are only mine,

And for the mistakes I am not to be blamed if I said such thing, it would be the greatest lie of which I would be much ashamed

Four long years of teamwork and solitude Condensed in a few hundred pages

That can hardly express my gratitude

To the ones I want to pay homage

I also take the opportunity herein

To use all forbidden literary inscriptions

That were considered scientific sins

By the man responsible for their deletion

Why not start with him

With whom I fought all these years

And yet stood by me through thick and thin

In spite of his 'writing' fears

'Your thesis is not a novel'

I often heard him say

But I could hardly stop 'flourishing'

To his complete and utter dismay

Yes, that is true:

Prof. Berger deserves a special 'thank you'

Above all for having helped

To get me through

Many extra thanks go to:

Bjorn Winkens, my roommate

Who has just discovered the joys of parenthood

For all his mathematical support

And for never being in a bad mood

Marga Doyle, a lady of impeccable style

Who basically adopted me and my son

For all her logistic back-up

And for making 'things' take an easy run

Mickey Chenault, my friend and confident

One thing is for sure:

Life in Maastricht would be more boring

Without her stories and allure

Frans Tan, a man of infinite patience

With equally the same amount of sapience

Who has never failed to mark his presence

My dear by-standers

Who make single-motherhood so easy:

In Maastricht, Bernard and Sophie,

In Bonn, the Maschke family

Just to give an idea of the role they play:

My son is so well looked after by these others

That he is now used to say

He has several mothers

Since I cannot keep rhyming ad infinutum

I come to an end of this short post-scriptum

And so, to finish it with a golden touch

I want to thank the one, who has always helped me

the most:

Justice where it is due,

Mother, when it comes to helping no one beats you! 